ZENTRUM FÜR BIODIVERSITÄT UND NACHHALTIGE LANDNUTZUNG

SEKTION

BIODIVERSITÄT, ÖKOLOGIE UND NATURSCHUTZ

CENTRE OF BIODIVERSITY AND SUSTAINABLE LAND USE

SECTION: BIODIVERSITY, ECOLOGY AND NATURE CONSERVATION

\title{
Mitochondrial genomes and the complex evolutionary history of the cercopithecine tribe Papionini
}

\author{
Dissertation \\ zur Erlangung des Doktorgrades \\ der Mathematisch-Naturwissenschaftlichen Fakultäten \\ der Georg-August-Universität zu Göttingen
}

vorgelegt von

Dipl. Biol.

Rasmus Liedigk

aus

Westerstede

Göttingen, September 2014 
Referent: PD Dr. Christian Roos

Korreferent: Prof. Dr. Eckhard Heymann

Tag der mündlichen Prüfung: 19.9.2014 


\section{Table of content}

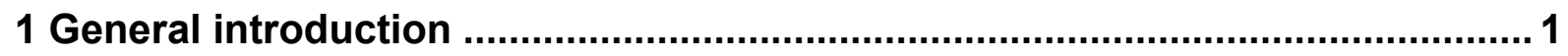

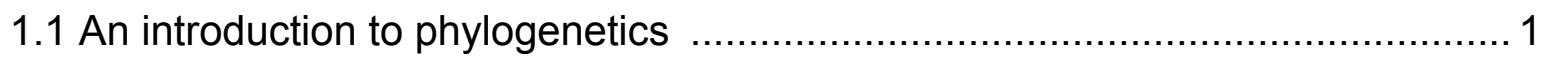

1.2 Tribe Papionini - subfamily Cercopithecinae …............................................. 3

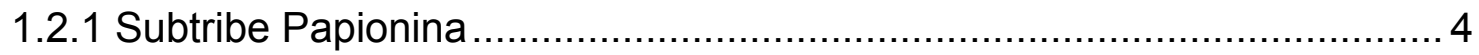

1.2.2 Subtribe Macacina, genus Macaca ………....................................... 5

1.3 Papionin fossils in Europe and Asia ....................................................... 7

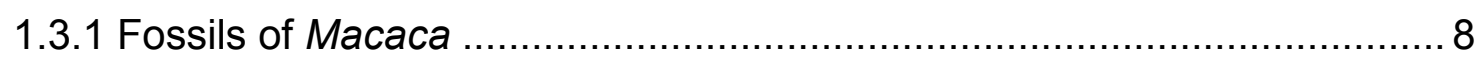

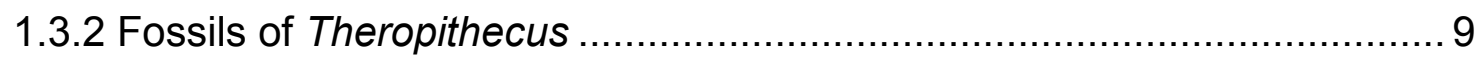

1.4 The mitochondrial genome and its application in phylogenetics ..................... 9

1.5 Divergence time estimation using molecular data......................................... 10

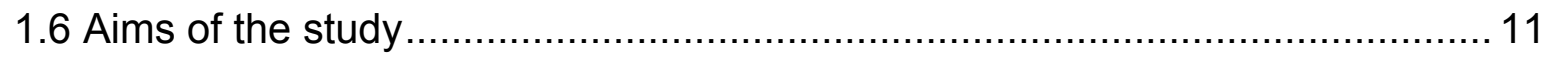

2 Mitogenomics of the Old World monkey tribe Papionini ...................................13

3 Baboon phylogeny as inferred from complete mitochondrial genomes.........41

4 Mitogenomic phylogeny and phylogeography of the common

long-tailed macaque, Macaca fascicularis fascicularis ..................................... 49

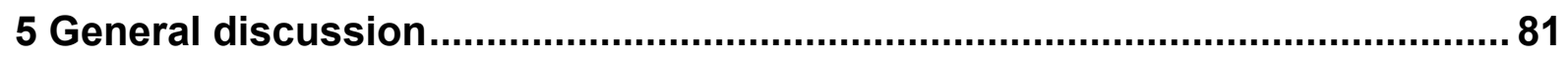

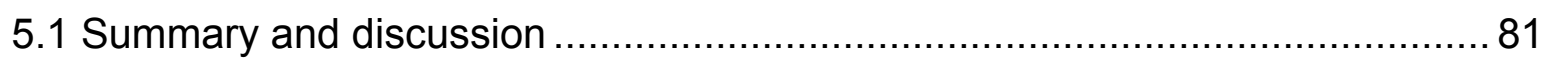

5.2 The mitochondrial genome and phylogenetic reconstructions ....................... 83

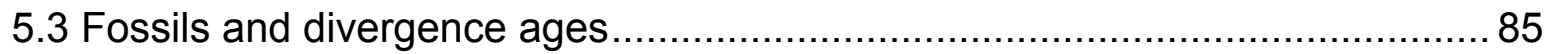

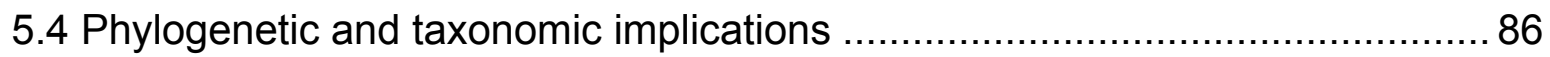

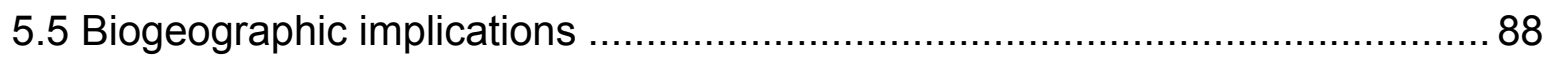

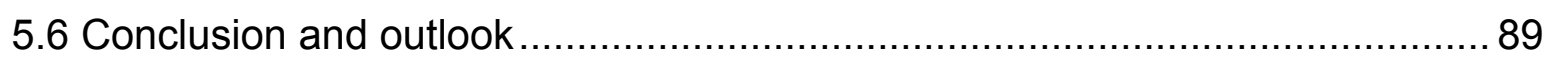

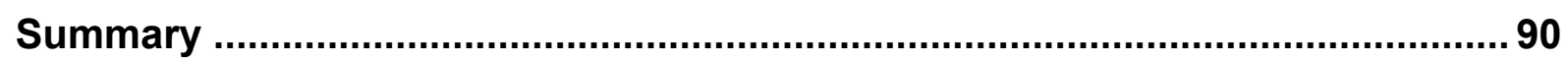

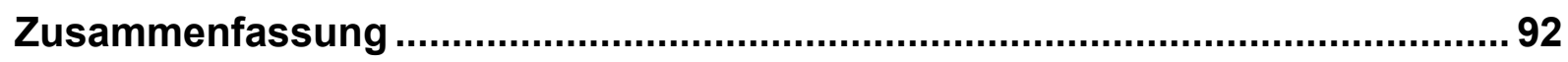

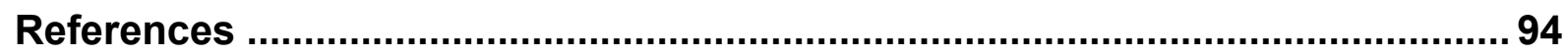

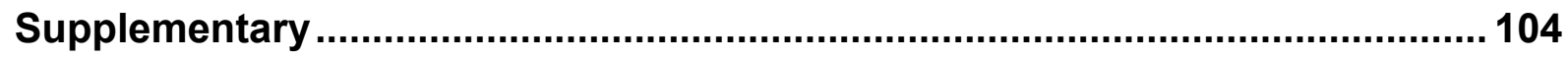

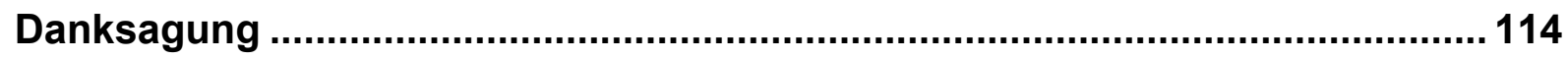

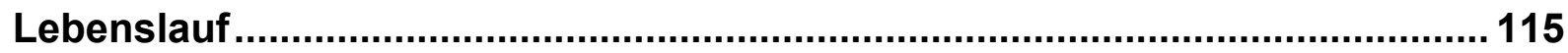




\section{Chapter 1}

\section{General Introduction}

\subsection{An introduction to phylogenetics}

First documented attempts of classifying the diversity of life go back to Aristotle around 2,400 years ago, who arranged organisms in a hierarchical way according to the complexity of their structure (Singer, 1931). Centuries later, in his Systema Naturae (1758) Carl Linnaeus was the first who categorised organisms by giving them genus and species names, and this binomial nomenclature is still applied in taxonomy and systematics nowadays (Beebee \& Rowe 2008). A century after Linnaeus Alfred Russel Wallace (1858) and Charles Darwin (1859) developed the theory of evolution independently from each other and it was also Darwin (1859) and slightly later Ernst Haeckel (1866) who primarily depicted systematic relationships in tree-like illustrations (tree of life). Since that time, innumerable phylogenetic studies attempted to resolve how species are related to each other. One of the most commonly used approaches to classify organisms is the cladistic method. Basic ideas about cladistic analyses go back to the work of Willi Hennig $(1950,1966)$ after whom the aim of phylogenetics is to describe taxa that share common ancestry and therefore form monophyletic groups (clades). To detect such clades one has to distinguish ancestral (plesiomorphic) and derived (apomorphic) traits. Monophyletic clades are defined by shared derived characters (synapomorphies), while similarities that evolved independently from each other (homoplasy, convergence) can lead, if falsely interpreted, to incorrect relationships of respective taxa.

For a long time only morphological and anatomical traits were investigated to disentangle phylogenetic relationships. Later on, at the beginning of the twentieth century, different molecular techniques were applied, e.g., chromosome analysis or protein structure analysis (Pauling et al., 1951; Sutton, 1903). However, these techniques had their limits for taxonomic analyses (Avery et al., 1944; Dobzhansky, 1937; Watson \& Crick 1953). In the 1970s and 1980s innovative methods like the Polymerase Chain Reaction (PCR) (Mullis et al., 1986) and Sanger sequencing 
(Sanger et al., 1977) were introduced and revolutionised the field of molecular biology, because with these techniques it was possible to use DNA-sequence information for phylogenetic reconstructions. Additional advances and improvements of techniques such as high-throughput sequencing and DNA capturing now allow to efficiently generate DNA sequence data not only from high-quality and -quantity material (e.g., tissue, blood) but also from highly degraded DNA and small amounts of raw material (e.g., faeces, hair follicle, museum material).

Until recently DNA-based studies in primate phylogenetics were mainly based on single loci, including relative short DNA fragments of a few hundreds of base pairs (bp) such as non-recombining mitochondrial DNA sequences (e.g., Cytochrome b; Andrews et al., 1998; Haus et al., 2013; Roos et al., 2003; Thinh et al., 2010a; Yoder et al., 1996; Zhang \& Ryder 1998; Ziegler et al., 2007). However, not all parts of the mammalian genome share the same mode of inheritance. While the $Y$ chromosome is inherited only via the paternal lineage, the mitochondrial genome is passed on only maternally. Autosomes and the $\mathrm{X}$ chromosome are inherited from both parents. Recently, it turned out that phylogenies inferred from different loci often result in alternative tree topologies (e.g., Tosi et al., 2000; 2002; Roos et al., 2011). Hence, the topology of a tree based on one gene or locus has always to be regarded with caution. Gene trees are not necessarily species trees (Avise, 2004) and therefore multi marker approaches are carried out to compare the results and to infer the true species relationships (multi locus coalescence approach) (Degnan \& Rosenberg 2009).

Phylogenetic analyses in primatology are generally of great interest, not only because primates are our closest relatives. Although primates are an extensively studied group, the field still offers astonishing new results concerning species delimitation and phylogenetic relationships. Even today there are still remote areas in Asia, Africa and South America which are understudied in terms of primate diversity and the respective phylogenetic relatedness of new or cryptic species. Molecular analyses are applied to solve phylogenetic relationships and to delimit species or taxonomic units. Such analyses can help to separate taxa that formerly were indistinguishable by morphological traits (cryptic species, e.g., mouse lemurs Microcebus spp., sportive lemurs Lepilemur spp., woolly lemurs Avahi spp., Andriaholinirina et al., 2006; Kappeler et al., 2005; Rabarivola et al. 2006; Tattersall, 2007; Zaramody et al. 2006). DNA sequence analyses led to the recent discovery of 
new primate species (e.g., Rhinopithecus strykeri, Trachypithecus cristatus selangorensis, Nomascus annamensis) (Geissmann et al., 2011; Roos et al., 2008; Thinh et al., 2010b) and even a new genus (Rungwecebus, Davenport et al., 2006).

Phylogenetic studies however are not only important for taxonomic reasons. Almost all fields of biological research benefit from clarified and robust phylogenies. Comparative evolutionary studies, e.g., in behavioural ecology, are only meaningful if the underlying phylogeny is reliable (Pozzi et al., 2013). In biomedical research phylogenetic information is essential, e.g., to infer how and when a certain immunological disposition evolved. Knowledge about the phylogenetic relationships among non-human primates, especially in the Cercopithecinae, is of special interest since they represent important biomedical model organisms (Haus et al., in press; Smith et al., 2007).

To reconstruct phylogenetic relationships reliably, knowledge about species diversity and precise species delimitation is needed. If taxa or respective specimens are erroneously allocated to a wrong species, respective phylogenies are confounded and can lead to false conclusions. A clear taxonomy and phylogeny is also indispensable for comprehensive biodiversity assessments which provide the basis for the IUCN Red List of Threatened Species and for effective species conservation plans.

The general aim of my thesis is to investigate the phylogenetic relationships within the important cercopithecine tribe Papionini (mainly Asian macaques and African mangabeys and baboons).

\subsection{Tribe Papionini - subfamily Cercopithecinae}

The Old World monkeys or Cercopithecidae represent the only extant family in the catarrhine primate superfamily Cercopithecoidea. As inferred from fossil data and molecular studies the Cercopithecoidea diverged from the Hominoidea between 2531 million years ago (Ma) (Chatterjee et al., 2009; Finstermeier et al., 2013; Perelman et al., 2011; Pozzi et al., 2011; Springer et al., 2012; Zalmout et al., 2010). The Cercopithecidae represent the most diverse family among all 16 primate families, including 23 genera and 159 species (Zinner et al., 2013). The Cercopithecidae consist of two subfamilies, the Colobinae (leaf monkeys) and the 
Cercopithecinae (cheek pouch monkeys) (Groves, 2001; Zinner et al., 2013), which diverged from each other between 13 and 23 Ma (Chatterjee et al., 2009; Finstermeier et al., 2013; Perelman et al., 2011; Springer et al., 2012). Colobines comprise two tribes, the African Colobini and the Asian Presbytini, and the cercopithecines consist of the tribes Cercopithecini and Papionini (Groves, 2001; Zinner et al., 2013), which diverged during the Middle and Late Miocene, respectively (Perelman et al., 2011). The origin of the Papionini is most likely Africa and most extant species still inhabit most regions of sub-Saharan Africa. The Papionini comprise two subtribes, the Macacina (mainly Asian macaques) and the Papionina (African mangabeys and baboons).

\subsubsection{Subtribe Papionina}

The Papionina comprise six genera and 23 species (Zinner et al., 2013). The genus Mandrillus (mandrill and drill) consist of two species (M. sphinx, M. leucophaeus), the Cercocebus mangabeys comprise seven species (C. galeritus, $C$. agilis, $C$. chrysogaster, C. sanjei, C. atys, C. lunulatus, C. torquatus), the Lophocebus mangabeys six species (L. albigena, L. osmani, L. johnstoni, L. ugandae, $L$. aterrimus, L. opdenboschi). The genera Rungwecebus and Theropithecus are monotypic and include the kipunji or highland mangabey ( $R$. kipunji) and the gelada ( $T$. gelada) respectively. The genus Papio currently comprises six species (P. papio, P. hamadryas, P. ursinus, P. anubis, P. kindae, P. cynocephalus).

The Papionina are geographically widespread and ecologically diverse (Harris, 2000). Among them one finds predominantly arboreal (Lophocebus, Rungwecebus) and terrestrial taxa (Cercocebus, Mandrillus, Papio, Theropithecus) (Geissmann, 2003; Zinner et al., 2013). Papionin taxa inhabit a variety of different habitat types which range from rainforest (Mandrillus, Cercocebus, Lophocebus, Rungwecebus) to savannah and semi-desert (Papio) and to mountainous regions (Theropithecus, Papio) (Zinner at al., 2013b). Within the Papionina, Papio is the only genus which extended its range beyond the borders of the African continent to southwestern Arabia.

The genus Papio (Erxleben, 1777) is mainly an African group inhabiting large regions of the sub-Saharan part of the continent. As indicated by fossil records (Jablonski \& Frost, 2010; Williams et al., 2012) and genetic studies (Keller et al., 
2010; Newman et al., 2004; Sithaldeen et al., 2009; Zinner et al., 2009; Zinner et al., 2013), the genus originated in southern Africa at around $2 \mathrm{Ma}$. The classification of the various Papio taxa is disputed. Groves (2001) and Grubb et al. (2003) proposed five species ( $P$. hamadryas, $P$. papio, $P$. anubis, $P$. cynocephalus, $P$. ursinus) while earlier studies combined all taxa in just a single species ( $P$. hamadryas or $P$. cynocephalus). The Kinda baboon was formerly considered a subspecies of the yellow baboon (P. cynocephalus kindae) (Groves, 2001; Kingdon 1997), but due to morphological (Frost et al., 2003) and genetic distinctiveness (Burrell, 2009; Zinner et al., 2009a) it was recently proposed as full species (Jolly et al., 2011; Zinner et al., 2013a). However, phylogenetic relationships among these six baboon taxa remain unclear. Phylogenetic studies based on molecular data yielded several cases of paraphyly and even polyphyly among Papio taxa. Zinner et al. (2009a) obtained seven major haplogroups, but phylogenetic relationships among them were not fully resolved. Further the obtained clades rather reflect the geographic distribution of respective taxa and do not correspond to the taxonomic classification (Keller et al., 2009; Zinner et al., 2009a; Zinner et al., 2009b).

Due to its vast distribution throughout savannah habitats and an evolution temporally in parallel to humans, baboons have been regarded as model taxon to understand early human dispersal scenarios (Garrigan \& Kingan, 2007; Jolly, 2001; Kopp et al. in press; Newman et al., 2004) and it is therefore of special interest to elucidate phylogenetic relationships within this genus.

\subsubsection{Subtribe Macacina, genus Macaca}

The genus Macaca (Lacépède, 1799) is, with the exception of Papio, the only papionin genus with extant members outside Africa (Evans et al., 1999; Groves, 2001; Zinner et al., 2013). Fossil data suggest that macaques arose in Northeast Africa approximately $7 \mathrm{Ma}$ and began their evolutionary diversification about $5.5 \mathrm{Ma}$, spreading north and eastward into Eurasia (Delson, 1975, 1980, 1996). During this range expansion, the genus diversified into distinct species groups that are variously defined along biogeographic, morphological and molecular lines (Delson, 1980; Riley, 2010; Tosi et al., 2003). Hence, macaques are one of the most successful extant primate radiations in terms of range expansion and diversity. The genus is highly speciose, is found in over 20 Asian countries and parts of Northern Africa and 
it covers an area of more than 5 million $\mathrm{km}^{2}$ (Tosi et al., 2003). The range of colonised habitats, from continents to islands, is unique among non-human primates (Abegg \& Thierry, 2002) and makes the genus Macaca an excellent example of adaptive radiation among primates (Riley, 2010).

The genus Macaca comprises 20 - 24 species depending on the classification of different authors (Groves, 2001; Tosi et al., 2003; Ziegler et al., 2007; Zinner et al., 2013) that are grouped into several species groups. Based on genital morphology of male macaques Fooden (1976) proposed four extant species groups that are: (1) the sylvanus-silenus group $(M$. sylvanus, $M$. silenus, $M$. nemestrina, Sulawesi macaques), (2) the fascicularis group ( $M$. fascicularis, M. mulatta, M. fuscata, $M$. cyclopis), (3) the sinica group (M. sinica, M. radiata, M. assamensis, M. thibetana) and (4) the monotypic $M$. arctoides group. By analysing cranial morphology Delson (1980) modified this classification by placing $M$. arctoides as a member of the sinica group and removing $M$. sylvanus from the silenus group to form a sister taxon to all other macaques. Taking both morphological and genetic data into account, Groves (2001) divided the genus into six species groups, (1) the monotypic M. sylvanus group, (2) the $M$. nemestrina group ( $M$. nemestrina, $M$. leonina, $M$. silenus, $M$. pagensis), (3) the Sulawesi group, (4) the $M$. fascicularis group ( $M$. fascicularis, $M$. arctoides), (5) the M. mulatta group (M. mulatta, M. cyclopis, M. fuscata) and (6) the M. sinica group (M. sinica, $M$. radiata, $M$. assamensis, $M$. thibetana). In contrast to Groves (2001), Zinner et al. (2013) separated M. fascicularis from M. arctoides and allocated both in monotypic groups, thus recognising a total of seven species groups.

Fooden $(1976,1980)$ proposed that macaques dispersed in three successive waves what he inferred from their present-day distribution. As the sylvanus-silenus (+ Sulawesi) lineage inhabits the most fragmented distribution, it was assumed to be the first that dispersed. The sinica-arctoides lineage with its moderately fragmented distribution was proposed to have dispersed secondly and the fascicularis (+ mulatta) lineage third as it has the most broadly continuous distribution (Fooden, 1976, 1980). Despite the general consensus about the above-mentioned lineages and their dispersal, the phylogenetic relationships among species and species groups have not been conclusive. A number of issues concerning relatedness and dispersal routes within Southeast Asia and the Sunda Shelf remain to be clarified.

Of special interest among macaques is the $M$. fascicularis group which is one of the youngest macaque lineages. The monotypic group (sensu Zinner et al., 2013) 
has beside rhesus macaques the largest distribution and it is found throughout southern Southeast Asia, the Sunda Shelf and beyond as far as the Philippines and the island of Timor. In the northern part of its range $M$. fascicularis is introgressed by the parapatric M. mulatta (Bonhomme et al., 2009; Tosi et al., 2000; Tosi et al., 2002; Tosi \& Coke 2007; Zinner et al., 2013). About $30 \%$ of the mainland M. fascicularis genome is of $M$. mulatta origin (Yan et al., 2011). Reconstruction of phylogenetic relationships based on molecular data showed a clear division into a continental and an insular fascicularis clade (Tosi et al., 2003), but when exactly both, the continental and insular populations diverged, is unclear. Inconsistencies of divergence times as inferred from mitochondrial and Y-chromosomal data, and the fact that both continental and insular genotypes are present on Sumatra support the hypothesis that both populations were in contact for several thousand years (Tosi et al., 2003; Tosi \& Coke 2007). The origin of the fascicularis group is unclear, but Delson (1980) suggested that macaques entered Sundaland during periods of low sea level. Pleistocene fossils from Java indicate that proto-fascicularis probably became isolated on Java and later extended its range again to the North (Delson, 1980). Further dispersal routes of $M$. fascicularis in Sundaland are not known and it is unclear whether the taxon extended its range to Timor by itself or whether humans introduced it there.

\subsection{Papionin fossils in Europe and Asia}

Having dispersed from Africa during the Late Miocene, several taxa of the cercopithecines existed in Europe during the Pliocene and the Early Pleistocene (Alba et al., 2014; Delson, 1974; Köhler et al., 2000). Fossil remains from outside of Africa that are assigned to recent papionin genera comprise solely macaques and members of the genus Theropithecus, whereas fossil remains of other extant papionin genera are only known in Africa. Cercocebus fossils have been recovered in South Africa, Kenya and Tanzania from Late Pliocene localities. Plio-Pleistocene remains of Lophocebus were also found in Kenya. Plio- and Pleistocene fossils, that were assigned to Papio are known from South and East Africa (Hartwig, 2002). In the following paragraph I give an overview about papionin fossils that were found outside of Africa. 


\subsubsection{Fossils of Macaca}

Fossil macaque remains have been found in southern and central Europe as well as in Asia. The European remains, mainly teeth and partial jaws, were designated to Macaca sylvanus prisca (Gervais, 1859; Delson, 1980) and were found in some cases in assemblages together with early colobines (Mesopithecus) (Alba et al., 2014; Delson, 1980). To date, probably the oldest macaque fossils outside Africa were discovered in Late Miocene localities in eastern Spain (Köhler et al., 2000) and northern Italy (Alba et al., 2014). Although not all European fossils have been assigned to the species level, most of them are considered to belong to the $M$. sylvanus lineage (Alba et al., 2014). Dental morphology of the European macaques do not differ much from M. lybica (Stromer, 1920) which was discovered in a Late Miocene excavation from northern Egypt, indicating affiliation to the same lineage ( $M$. sylvanus). Taking together the information about earliest macaque fossils in Europe, it is assumed that a dispersal of the genus, and cercopithecines in general, out of Africa took place at the Miocene - Pliocene boundary (Alba et al., 2014). Recently, a 6.5 - 8 million-years-old guenon fossil (Cercopithecini) was discovered in Arabia (Gilbert et al., 2014). Although guenons belong to the sister tribe of the Papionini, this finding indicates that dispersal of cercopithecines out of Africa might have occurred earlier than previously thought.

Compared to the fossil record of Europe, macaque associated remains from Asia are rather scarce (Delson, 1980). The first cercopithecid fossils in Asia were, as in Europe, colobines and the oldest fossil associated with Macaca were found in China ( 4 Ma) (Alba et al., 2014) and in northern India ( 3 Ma) (Delson, 1980). All other macaque fossils from Asia are from the Pleistocene. Dental remains from the Early Pleistocene that were found in China were named $M$. anderssoni and $M$. robusta (Schlosser, 1924; Young, 1934), whereas the former is similar to the modern M. thibetana and M. arctoides (Delson, 1980). The youngest Chinese macaque fossil is from the Late Pleistocene and can be referred to M. mulatta. A Middle Pleistocene fossil that is also associated with $M$. thibetana and $M$. arctoides was found in northern Vietnam (Jouffroy, 1959). Fossil macaques from Java (Middle Pleistocene) are not precisely identified but may be associated with $M$. fascicularis or $M$. 
nemestrina (Delson, 1980). Further remains have been recovered in South Korea where macaques are absent nowadays.

\subsubsection{Fossils of Theropithecus}

The fossil record of Theropithecus reveals that the genus has been quite abundant in the Plio-Pleistocene of Africa (Delson, 1993; Delson et al., 1993; Roberts et al., 2014; Rook et al., 2004), whereas it is nowadays restricted to the highlands of Ethiopia (Jablonski \& Frost, 2010). Theropithecus fossils, which have been recovered from north-eastern Ethiopia, were allocated to $T$. oswaldi cf. darti. These remains have been dated to between 3.6 and 3.8 Ma and were found in an assemblage with other cercopithecines and colobines (Frost et al., 2014). During the Early Pleistocene, much later than macaques, Theropithecus extended its range from Africa, via the Near East ( 1.4 Ma, Belmaker, 2002; Rook et al., 2013) to India ( 1.0 Ma), southern Italy and Spain ( 1.0 Ma) (Delson, 1993; Delson et al., 1993; Gibert et al., 1995; Gupta \& Sahni, 1981; Pickford, 1993; Roberts et al., 2014; Rook et al., 2004, 2013). Theropithecus remains from Southeast Asia are not reported.

\subsection{The mitochondrial genome and its application in phylogenetics}

Most eukaryotic cells contain mitochondria in the cytoplasm. These organelles, which serve as chemical power supplies for the cell, comprise their own DNA. The mitochondrial DNA (mtDNA) evolves independently from the nuclear DNA (nDNA) and is inherited only via the maternal lineage since sperm cells do not contribute any mitochondria to the zygote. The circular, double-stranded mtDNA molecule in vertebrates is around 16.5 kilobases $(\mathrm{kb})$ in length and consists of 37 genes coding for two rRNA, 22 tRNAs and 13 proteins and includes also the non-coding control region (Beebee \& Rowe, 2008; Wolstenholme, 1992).

Analysing mtDNA in the context of phylogenetic studies provides several advantages. Each mitochondrion contains two to ten copies of mtDNA and each cell contains up to several thousand mitochondria, hence the number of copies of mtDNA per cell is much higher than that of nDNA (Wiesner et al., 1992). This is of particular importance when working with degraded DNA as found e.g. in faeces or ancient 
material like museum specimens or sub-fossil material. Another advantage is that the mutation rate in vertebrate mtDNA is five to ten times higher than in nDNA, which makes it a useful molecular marker to study closely related taxa and inter-specific relationships (Beebee \& Rowe, 2008).

As mentioned earlier, mtDNA is only inherited maternally. Since most primate taxa live in female philopatric societies, in which males disperse and females stay in their natal groups, mtDNA can reveal insights into genetic differences among regional populations (Smith et al., 2007). Furthermore, the instance of maternal inheritance can also help to trace back the geographical origin of respective taxa (Avise, 2004), at least on an intra-specific level where splits between taxa are relatively young.

Generally, the mutation rate in mtDNA is higher than in nDNA, but the nucleotide substitution rate also varies within the mitochondrial genome. Therefore different parts of the mitochondrial genome have been used to reconstruct phylogenetic relationships among primate taxa. Several studies used the less variable cytochrome b gene (Andrews et al., 1998; Haus et al., 2013; Roos et al., 2003; Thinh et al., 2010b; Yoder et al., 1996; Zhang \& Ryder 1998; Ziegler et al., 2007) to estimate affiliations among closely related primate taxa. In other studies that focused more on genetic diversity within and among populations, the highly variable control region was investigated (e.g., Ebenau et al., 2011; Yang et al., 2012).

While working with mtDNA care has to be taken not to amplify nuclear pseudogenes, the so-called numts (nuclear mitochondrial sequences). Numts are copies of mtDNA that are integrated into the nDNA where they evolve independently from the true mtDNA. The amplification of these numts can result in confounded phylogenies (Thalmann et al., 2004) and therefore have to be avoided. The chance to amplify numts is relatively low when using DNA extracted from faecal samples or museum specimens since nDNA is normally highly degraded in such material.

\subsection{Divergence time estimation using molecular data}

DNA sequence data can be used to estimate divergence times of certain lineages. The underlying concept of a molecular clock (Zuckerkandl \& Pauling, 1965) means that the nucleotide substitution rate within a certain DNA region is constant among all 
lineages. The degree of divergence between two lineages is assumed to be directly proportional to the time since divergence. In the meantime it became clear that evolutionary rates vary among different taxa depending on e.g. population size, body size, metabolic rate and generation time (Martin \& Palumbi, 1993), especially when deep splits are considered. Therefore a relaxed molecular clock approach, which addresses these differences, is in most cases applied instead of a strict molecular clock model. Regardless of which clock model is favoured, all molecular phylogenies, which incorporate divergence date estimation, need to be calibrated. Normally this is done with fossils. Therefore the respective fossil record has to be browsed for specimens that reliably document the occurrence of a particular taxon in a certain time range. These data can be used to fix respective nodes in a phylogeny of interest, what is essential for reliable time estimations.

\subsection{Aims of the study}

On the basis of complete mitochondrial genome sequences I aim to reconstruct phylogenetic relationships on different taxonomic levels within the Papionini. By investigating inter- and intra-generic as well as intra-specific relationships a broad time range of the evolutionary history of the Papionini is taken into account.

1. In the first study (chapter 2) I combine representatives from all genera and species groups of the Papionini (with the exception of Rungwecebus) in one comprehensive phylogeny and estimate divergence times. In this context relationships within the Mandrillus - Cercocebus and within the Theropithecus Lophocebus - Papio clade, as well as relationships among macaque species groups are of special interest since previous studies depicted contradicting relationships. For this study, I did all laboratory work, analysed data together with Markus Brameier (MB), Christian Roos (CR) and Dietmar Zinner (DZ), and wrote the paper together with $\mathrm{CR}$ and $\mathrm{DZ}$.

2. In the second study (chapter 3) we investigate affiliations among different populations and species of the African genus Papio to test whether complete mtDNA genomes reveal a better resolution of phylogenetic relationships than previous analyses using only short mtDNA sequences. For this study, I did laboratory work 
together with Jenny Wertheimer, and analysed data and wrote the paper together with Linn F. Groeneveld, DZ and CR.

3. In the third study (chapter 4) we analyse the intra-specific relationships within Macaca $f$. fascicularis by reconstructing a mitogenomic phylogeny using samples from throughout the subspecies' range. The split between mainland and Sundaland populations as well as possible dispersal routes are of special interest. The study incorporates sequence data inferred from traditional Sanger sequencing as well as from a DNA capture method followed by high-throughput sequencing. Hence, we are able to compare the accuracy of both methods. For this study, I did laboratory work together with Jakob Kolleck (JK) and Kai Böker (KB), analysed data together with $\mathrm{JK}, \mathrm{KB}, \mathrm{MB}, \mathrm{DZ}$ and $\mathrm{CR}$, and wrote the paper together with $\mathrm{DZ}$ and $\mathrm{CR}$. 


\section{Chapter 2}

\section{Mitogenomics of the Old World monkey tribe Papionini}

Rasmus Liedigk ${ }^{1 *}$, Christian Roos ${ }^{1,2}$, Markus Brameier $^{1}$, Dietmar Zinner ${ }^{3}$

${ }^{1}$ Primate Genetics Laboratory, German Primate Center, Leibniz Institute for Primate Research, Kellnerweg 4, 37077 Göttingen, Germany

${ }^{2}$ Gene Bank of Primates, German Primate Center, Leibniz Institute for Primate Research, Kellnerweg 4, 37077 Göttingen, Germany

${ }^{3}$ Cognitive Ethology Laboratory, German Primate Center, Leibniz Institute for

Primate Research, Kellnerweg 4, 37077 Göttingen, Germany

*Correspondance: rliedigk@gmx.de

Accepted for publication in BMC Evolutionary Biology, 25 July 2014. 


\section{Abstract}

\section{Background}

The evolutionary history of the Old World monkey tribe Papionini comprising the genera Macaca, Mandrillus, Cercocebus, Lophocebus, Theropithecus, Rungwecebus and Papio is still matter of debate. Although the African Papionini (subtribe Papionina) are generally considered to be the sister lineage to the Asian Papionini (subtribe Macacina), previous studies based on morphological data, nuclear or mitochondrial sequences have shown contradictory phylogenetic relationships among and within both subtribes. To further elucidate the phylogenetic relationships among papionins and to estimate divergence ages we generated mitochondrial genome data and combined them with previously published sequences.

\section{Results}

Our mitochondrial gene tree comprises 33 papionins representing all genera of the tribe except Rungwecebus. In contrast to most previous studies, the obtained phylogeny suggests a division of the Papionini into three main mitochondrial clades with similar ages: 1) Papio, Theropithecus, Lophocebus; 2) Mandrillus, Cercocebus; and 3) Macaca; the Mandrillus + Cercocebus clade appears to be more closely related to Macaca than to the other African Papionini. Further, we find paraphyletic relationships within the Mandrillus + Cercocebus clade as well as in Papio. Relationships among Theropithecus, Lophocebus and Papio remain unresolved. Divergence ages reveal initial splits within the three mitochondrial clades around the Miocene/Pliocene boundary and differentiation of Macaca species groups occurred on a similar time scale as those between genera of the subtribe Papionina.

\section{Conclusion}

Due to the largely well-resolved mitochondrial phylogeny, our study provides new insights into the evolutionary history of the Papionini. Results show some contradictory relationships in comparison to previous analyses, notably the paraphyly within the Cercocebus + Mandrillus clade and three instead of only two major mitochondrial clades. Divergence ages among species groups of macaques are 
similar to those among African Papionini genera, suggesting that diversification of the mitochondrial genome is of a similar magnitude in both subtribes. However, since our mitochondrial tree represents just a single gene tree that most likely does not reflect the true species tree, extensive nuclear sequence data is required to illuminate the true species phylogeny of papionins and to trace possible ancient hybridization events among lineages.

\section{Keywords}

Phylogeny, Divergence ages, mtDNA, Primates, Macaques, Baboons

\section{Background}

It is well recognized that mitochondrial (mtDNA) phylogenies are not necessarily congruent with the phylogeny of the respective taxa or phylogenies based on a set of nuclear genes (e.g. [1-3]). Reasons for the incongruence are manifold, e.g., different inheritance pathways, divergent selection pressures, and most prominent, incomplete lineage sorting and horizontal gene flow (e.g. [4,5]). On the other hand, if mtDNA and nuclear (nDNA) phylogenies are congruent this could be a strong indication that the single underlying gene tree is congruent with the species tree. Furthermore, in many species analyses of mtDNA relationships provide a better spatial resolution, thus contributing to phylogeographical inferences $[3,6]$. Therefore, analyses of both, mtDNA and nDNA, are necessary for a comprehensive understanding of the evolutionary history of taxa and for a robust reconstruction of complex phylogenies.

Among primates, incongruences are reported for several taxa within the Old World monkey tribe Papionini (e.g. [7-14]). The Papionini tribe diverged from its sister lineage, the Cercopithecini, around 11.5 million years ago (Ma) [15] and is comprised of the subtribe Papionina, with the genera Papio, Mandrillus, Theropithecus, Cercocebus, Rungwecebus and Lophocebus, and the subtribe Macacina, with the genus Macaca [16]. While all available nDNA data and respective gene trees are congruent and strongly support this division $[15,17,18]$, recent studies applying 
mtDNA genome data suggest the Mandrillus + Cercocebus clade to be closer related to Macaca $[19,20]$, thus indicating paraphyly of Papionina in the mtDNA gene tree.

The African origin of the tribe is broadly accepted [16,21-25] and the fossil record indicates a Late Miocene dispersal out of Africa into Eurasia for some lineages. Remains of macaques have been found in southern, western and central Europe [26,27], whereas fossil macaques from Asia are documented but rather scarce [26]. Fossils of Theropithecus have been recovered from the Iberian Peninsula as well as from India [28-34]. The six genera of Papionina are found today exclusively on the African continent, with the exception of the hamadryas baboon, which occurs in both northeastern Africa and the southwestern Arabian Peninsula [16,25]. In contrast, members of the subtribe Macacina are distributed over large regions of South, Southeast and East Asia with the exception of Barbary macaques, which are found in Northwest Africa. Based on morphological characters, the subtribe Papionina is divided into six relatively heterogeneous genera, while the Asian lineage consists of only one highly speciose genus (Macaca), which is divided into several species groups $[16,23,26,35]$.

The tribe comprises 45 species [36], exhibiting a great variety of morphologies from more slender representatives like the crested mangabeys to more robust forms like baboons, mandrills and drills. The genus Macaca is divided into species groups, but the number and the composition of these species groups have been a matter of debate for decades $[23,26,35]$. Based on the morphology of male genitals Fooden [35] proposed four species groups comprising a $M$. silenus-M. sylvanus, a $M$. fascicularis, a $M$. arctoides and a $M$. sinica group, with a total of 19 species. Delson [26] also proposed four species groups but moved $M$. arctoides into the $M$. sinica group and separated $M$. sylvanus from the $M$. silenus lineage into its own group. Combining morphological and genetic data, Groves [23] proposed a classification into six species groups with a total of 20 species: (1) the monotypic M. sylvanus group, (2) the M. nemestrina group, (3) the Sulawesi group, (4) the M. fascicularis group, (5) the $M$. mulatta group and (6) the $M$. sinica group. In the most recent classification the genus Macaca consists of 22 species, which are divided into seven species groups [16], among them three monotypic species groups: (1) M. sylvanus group, (2) M. arctoides group and (3) M. fascicularis group, and four polytypic groups: (4) Sulawesi group, (5) M. mulatta group, (6) M. sinica group and (7) $M$. 
silenus group. Although the monophyly of the macaques was confirmed in several studies $[23,26,35,37,38]$, relationships among and within the species groups are still disputed [37-40].

Similarly, within the African Papionina, relationships among genera and species are only partly resolved [41]. Findings based on morphological traits were often discordant with results from molecular studies. While early morphological analyses supported the monophyly of the mangabeys [42,43], more recent morphological [4446] and molecular studies [17,47,48] suggested diphyly of mangabeys, with Lophocebus clustering with Papio and Theropithecus, while Cercocebus forms a clade with Mandrillus. The kipunji (Rungwecebus kipunji), earlier described as a member of Lophocebus [49], was recently placed in its own genus [50]. Subsequent genetic studies confirmed the diphyly of Lophocebus and Cercocebus, and in addition showed a close relationship of Rungwecebus to Papio [10,50,51]. Concerning Papio, genetic analyses revealed seven well-supported mtDNA haplogroups, but these were not congruent with the six recognized species of the genus [11,42,52-54]. Likewise, for the Mandrillus + Cercocebus clade a mtDNA study indicated paraphyly of Cercocebus with at least one species (C. torquatus) being more closely related to Mandrillus than to its congenerics [12], while nuclear gene trees suggest reciprocal monophyly of both genera [14,15]. Previous morphological studies noted some similarities between Mandrillus, Cercocebus and Macaca. Fleagle and McGraw [45,55] studied postcranial features of Mandrillus, Cercocebus, Lophocebus and Papio and compared them with respective data of one macaque species (M. nemestrina). Most characters of Mandrillus and Cercocebus did not differ from those of $M$. nemestrina, and were therefore interpreted to be primitive among papionins, whereas just one of the investigated traits in $M$. nemestrina did not differ from that of Lophocebus, Papio and Theropithecus [45,55]. Furthermore, although it is widely accepted that Lophocebus and Theropithecus cluster together with a clade consisting of Papio and Rungwecebus, the branching pattern among these lineages is unresolved $[14,19,20,56]$.

It has recently been shown that the use of complete mtDNA genome sequences provide better statistical support in phylogenetic reconstructions when compared to analyses based on single genes or partial genomes (e.g. [57-60]). In our study we generated new mtDNA genome data of Macaca species and combined it with 
respective data of other Papionini from GenBank to reconstruct a robust mtDNA gene tree of papionin primates and to estimate respective divergence ages. We were particularly interested to obtain further information concerning the branching pattern among papionin genera and among all seven species groups of the genus Macaca and to provide comprehensive data for further comparative molecular studies.

\section{Results}

We sequenced complete mtDNA genomes from eight macaques representing all seven macaque species groups: $M$. sylvanus $-M$. sylvanus group, $M$. silenus $-M$. silenus group, $M$. tonkeana - Sulawesi group, $M$. thibetana - M. sinica group, $M$. mulatta/China and $M$. mulatta/India - M. mulatta group, $M$. fascicularis/Vietnam - $M$. fascicularis group, and $M$. arctoides - $M$. arctoides group. A BLAST-search in GenBank showed that our newly generated sequences matched almost perfectly with available orthologs. The full-length genome sequences consisted of 13 proteincoding genes, 2 rRNA genes, 22 tRNA genes and the control region. The initial alignment comprised 38 sequences and had a length of 16,966 bp. After indels and poorly aligned positions were removed the alignment comprised 15,685 bp including 6,986 informative sites. The alignment is available for download (Additional file 1 [61]).

The phylogenies as obtained from maximum-likelihood (ML) and Bayesian analyses are mainly identical and most branching patterns are strongly supported (Figure 1). Likewise, the Densitree [62] depicting the posterior distribution of the 25,000 trees as inferred from the Bayesian divergence age analysis in BEAST suggests the most frequent tree topology to be identical to that obtained from $M L$ and Bayesian analyses (Figure 2). According to divergence age estimations using auto-correlated and uncorrelated clock models, the Old World monkeys (Cercopithecoidea) diverged from the Hominoidea between 24 and $27 \mathrm{Ma}$ (for 95\% credibility intervals see Additional file 2: Table S1). In the Early Miocene, the two subfamilies of the Cercopithecidae, Colobinae and Cercopithecinae, separated, and the latter further split into Cercopithecini and Papionini between 11 and $16 \mathrm{Ma}$. Our analysis revealed three major clades within the Papionini which diverged 9-13 Ma. Interestingly, the Mandrillus + Cercocebus clade forms a sister lineage to Macaca (ML bootstrap value [BP]: 100\%; Bayesian posterior probability [PP]: 1.0) and does not cluster with the 
second major African papionin clade comprising Papio, Lophocebus and Theropithecus (BP: 100\%; PP: 1.0). Since Mandrillus and Cercocebus show a shift in $A / C$ content similar to macaques (Additional file 3: Figure $S 1$ ), which could lead to an artificial clustering [63], we repeated our analysis with a modified dataset (dataset 2) that corrects for this shift. Accordingly in this second alignment we masked positions that contain both an Adenin and Cytosin with an "M". The resulting overall branching pattern and specifically the phylogenetic position of the Mandrillus + Cercocebus clade among papionins were identical to those obtained from the original dataset (Additional file 4: Figure S2). To further test for alternative positions of the Mandrillus + Cercocebus clade among papionins, we performed alternative tree topology tests, which revealed that all alternative options are statistically rejected (Figure 3).

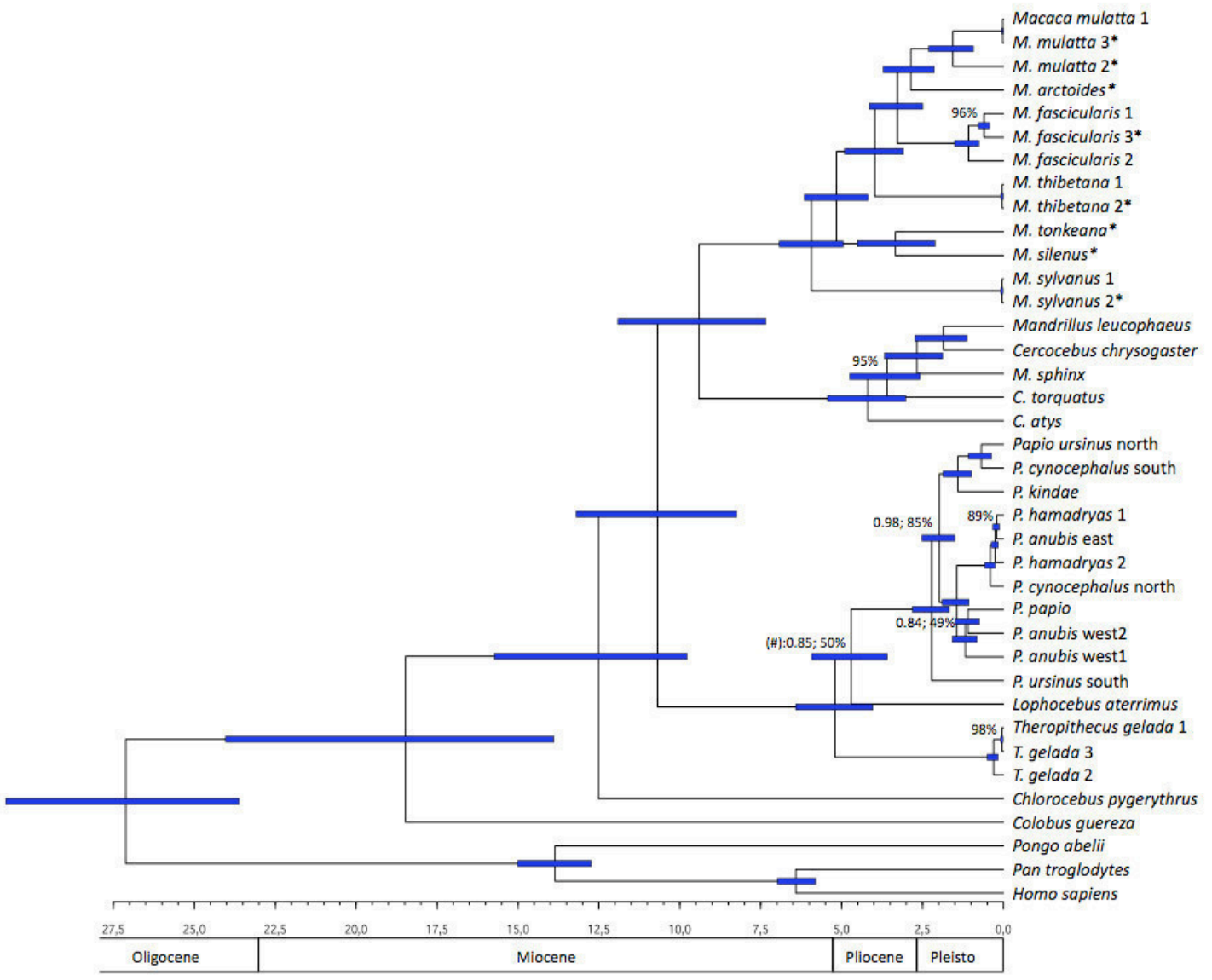

Figure 1 Ultrametric tree of the Papionini and outgroup taxa as inferred from mtDNA dataset 1. Tree topologies as inferred from Bayesian (MrBayes) as well as from ML (RAxML) estimation were identical with one exception: At one node (labelled with \#) the ML tree indicates Lophocebus as sister lineage to the Papio + Theropithecus clade (not depicted). All unlabelled branches show ML BP of $100 \%$ and Bayesian PP of 1.0 . Values below are indicated at respective nodes. Blue bars indicate $95 \%$ credibility intervals of divergence ages. Time scale shows million years before present. For information about taxa and samples see Additional file 7: Table S2. * = sequences were newly generated in this study. 


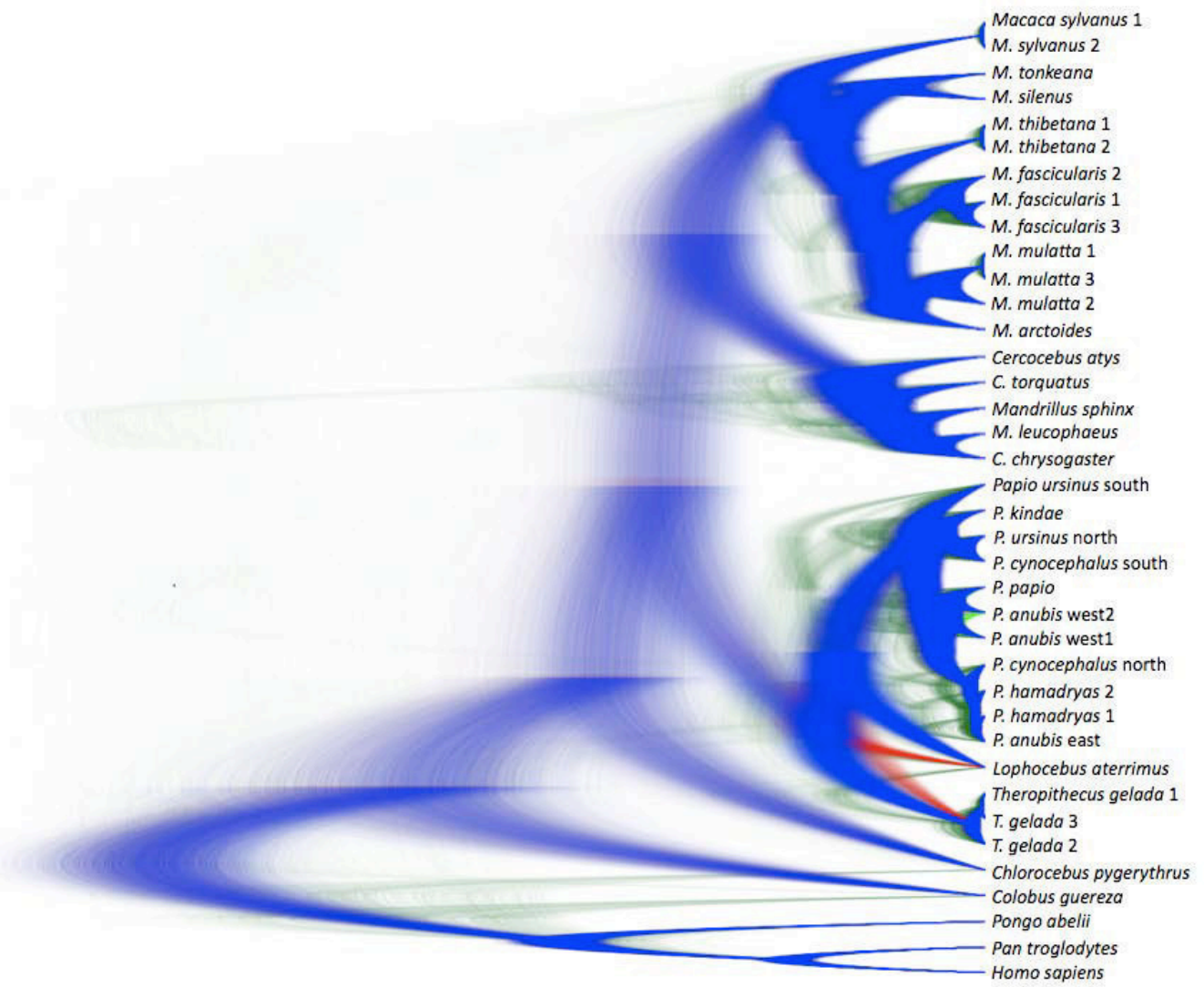

Figure 2 Densitree showing the posterior probability of 25,000 trees taken from the Bayesian divergence age analysis in BEAST. Blue represents the most frequent tree topology, red represents the second and green the third most frequent topology.

Within the Mandrillus + Cercocebus clade, members of both genera do not form reciprocally monophyletic clades. In dataset $1 \mathrm{C}$. atys is the first lineage to split off (4.2-4.9 Ma) followed by C. torquatus (3.6-4.3 Ma), while M. sphinx represents a sister lineage to $C$. chrysogaster and M. leucophaeus (BP: 100\%; PP: 1.0) which separated from them 2.7-3.4 Ma. The latter two diverged 1.9-2.6 Ma. The Bayesian analysis of dataset 2 shows the same topology, but partly with low support (PP: 0.56) while the $\mathrm{ML}$ analysis of dataset 2 suggests a possible clade consisting of $C$. atys and $C$. torquatus but only weakly supported (BP: 49\%) (Additional file 4: Figure S2).

Within the second African papionin clade, the branching pattern among the three genera Papio, Theropithecus and Lophocebus is not well resolved. While in the Bayesian analysis of the original dataset, Theropithecus is suggested as the first lineage to diverge (PP: 0.85), ML analysis of dataset 1, as well as ML and Bayesian 
analyses of dataset 2 indicates a Theropithecus + Papio clade to the exclusion of Lophocebus. Node supports for respective branching patterns are low (dataset 1, BP: $50 \%$; dataset 2, PP: 0.89; BP: $83 \%$ ). Similarly, the Densitree indicates Lophocebus + Papio as the most frequent clade, while the second most frequent clade is formed by Theropithecus and Papio. Estimated divergence ages suggest that respective splitting events occurred during a short time period around $5 \mathrm{Ma}$. Among Papio representatives the tree topology is identical and divergence ages are similar as previously reported [54], depicting paraphyletic relationships in $P$. ursinus, $P$. cynocephalus and $P$. hamadryas, and polyphyletic relationships in $P$. anubis. According to estimated divergence ages, splitting events within Papio started around $2 \mathrm{Ma}$. Among macaques, Macaca sylvanus diverged first, 5.9-6.3 Ma. Subsequently the Asian macaques radiated and successively split up into the six Asian species groups. The $M$. silenus + $M$. tonkeana $(M$. tonkeana as representative of the Sulawesi group) clade separated from the remaining macaques between 5.2-5.9 Ma and further segregated into two species groups (3.2-4.6 Ma). Among the remaining macaques, $M$. thibetana (as representative of the $M$. sinica group) diverged between 3.9-5.0 Ma from a M. fascicularis + M. arctoides + M. mulatta clade. Within the latter, $M$. fascicularis split off first (3.2-4.6 Ma) whereas $M$. arctoides separated from the $M$. mulatta clade slightly later (2.7-4.3 Ma). Within $M$. fascicluaris and $M$. mulatta we found relatively ancient splitting events of 1.1-2.2 Ma and 1.4-2.9 Ma.

\section{Discussion}

The application of complete mtDNA genome sequences revealed highly supported branching patterns for most of the investigated papionin lineages. The mtDNA gene tree as well as estimated divergence ages are broadly consistent with those reported in previous studies, but also show some remarkable, but not unexpected discordances to recent nDNA studies $[15,19,20,54,64,65]$.

The major findings of our analysis are: 1) a sister grouping of Macaca and the Mandrillus + Cercocebus clade, 2) paraphyly within the Mandrillus + Cercocebus clade, 3) unresolved relationships among Papio, Lophocebus and Theropithecus, and 4) similar divergence ages among Macaca species groups and papioninan genera. Furthermore, our phylogenetic reconstruction reveals highly supported branching patterns among the seven Macaca species groups, which are largely in 
agreement with most previous studies, (e.g. $[15,37,66]$ ). The only exception is the phylogenetic position of $M$. arctoides, which is here strongly supported as the sister lineage to the $M$. mulatta group. This finding is not surprising given the evidence that $M$. arctoides is the result of hybridization between ancestral forms of the $M$. sinica and $M$. mulatta groups $[37,66]$.

Divergence dates are mostly consistent regardless of the software (BEAST or PhyloBayes) and clock model (auto-correlated or uncorrelated) that were applied (Additional file 2: Table S1, Additional file 5: Figure S3, Additional file 6: Figure S4). Our estimation indicates a separation of African and Asian macaques around $6 \mathrm{Ma}$ which is in line with Alba et al. [27], who, based on fossil data, proposed a macaque dispersal from Africa into Eurasia by the Late Miocene (5.3-5.9 Ma). Generally, our divergence age estimations reveal a stepwise but rapid radiation of macaque genera between 5.9 and $2.7 \mathrm{Ma}$ in Asia, which is in agreement with the appearance of the earliest Macaca-like fossil in Asia which was found in the Yushe Basin (China) from about $4 \mathrm{Ma}$ [27]. At that time two of the six main lineages of Asian macaques were already established as indicated by our divergence age estimations. To further test possible dispersal scenarios in Southeast Asia and especially in Sundaland further taxa of the species groups from different locations have to be included in future analyses.

We found the Mandrillus + Cercocebus clade to be more closely related to the macaques than to other African Papionina, a pattern also reported by Finstermeier et al. [19] and Pozzi et al. [20]. However, in contrast to Finstermeier et al. [19] alternative tree topology tests with our data were clearly rejected (Figure 3), which most likely can be explained by the increased taxon sampling in our study (33 sequences this study, 11 sequences in Finstermeier et al. [19]), because it is known to reduce phylogenetic error [67-70]. Moreover, since we controlled for the observed shift in A/C content, the Mandrillus + Cercocebus clade might be indeed more closely related to Macaca than to the other African papionins, at least if we consider mtDNA. This finding, however, is contradictory to relationships based on recent nuclear studies, which found the Macacina and Papionina to be reciprocally monophyletic $[15,18]$. Perelman et al. [15] found this branching pattern in a concatenated dataset of 54 nDNA loci (BP: 100\%) as well as in six separately analysed subsets, of which four are similarly highly supported (BP: 97-100\%). Likewise, the presence/absence 
pattern of Alu integrations revealed no conflicting integrations, suggesting reciprocal monophyly of both clades [18] and Springer et al. [71], analysing a combined dataset of mtDNA and nDNA sequences, found the same pattern. Interestingly, comparative morphological studies investigating postcranial traits of African Papionina (Mandrillus, Cercocebus, Lophocebus and Papio) and one species of Macaca (M. nemestrina) suggest some similarities between Mandrillus + Cercocebus and the macaque [45,55]. However, since only one macaque species was included in the analysis, results concerning the relationship of Mandrillus + Cercocebus to Macaca have to be considered with caution. The question is whether the similarities between Mandrillus, Cercocebus and $M$. nemestrina are due to the plesiomorphy of the traits as suggested by Fleagle \& McGraw [45,55] or whether they result from convergent adaptations to similar ecological niches since Mandrillus, Cercocebus and $M$. nemestrina are predominantly forest dwelling terrestrial primates [72,73]. Given that nDNA phylogenies (e.g. [15]) may reflect the true species relationships more reliably than mtDNA phylogenies with Macaca being basal to the Papionina, we would assume that morphological similarities result from convergent adaptation. In contrast, the present mtDNA phylogeny would rather accord to the assumption that the shared morphological features are primitive.

Inconsistencies of mitochondrial and nuclear phylogenies are often explained by incomplete lineage sorting or ancient hybridization [5,19,37,59,60,74,75]. At the moment, we cannot determine if one or both phenomena affected the suggested phylogenetic relationships. A possible scenario based on hybridization could be that ancestral representatives of the Mandrillus + Cercocebus clade were indeed more closely related to ancestral macaques, but were later introgressed by an ancestor of the Papio + Theropithecus + Lophocebus clade, resulting in nuclear swamping. Hybridization seems to be common among extant papioninan taxa, even between genera $[11,12,76,77]$. It is therefore likely that hybridization and introgression also occurred among the ancestral papioninan lineages which lead to the observed incongruence between nDNA and mtDNA phylogenies. However, as mentioned above, incomplete sorting of mitochondrial lineages in these taxa is also a plausible explanation for the observed relationships. 


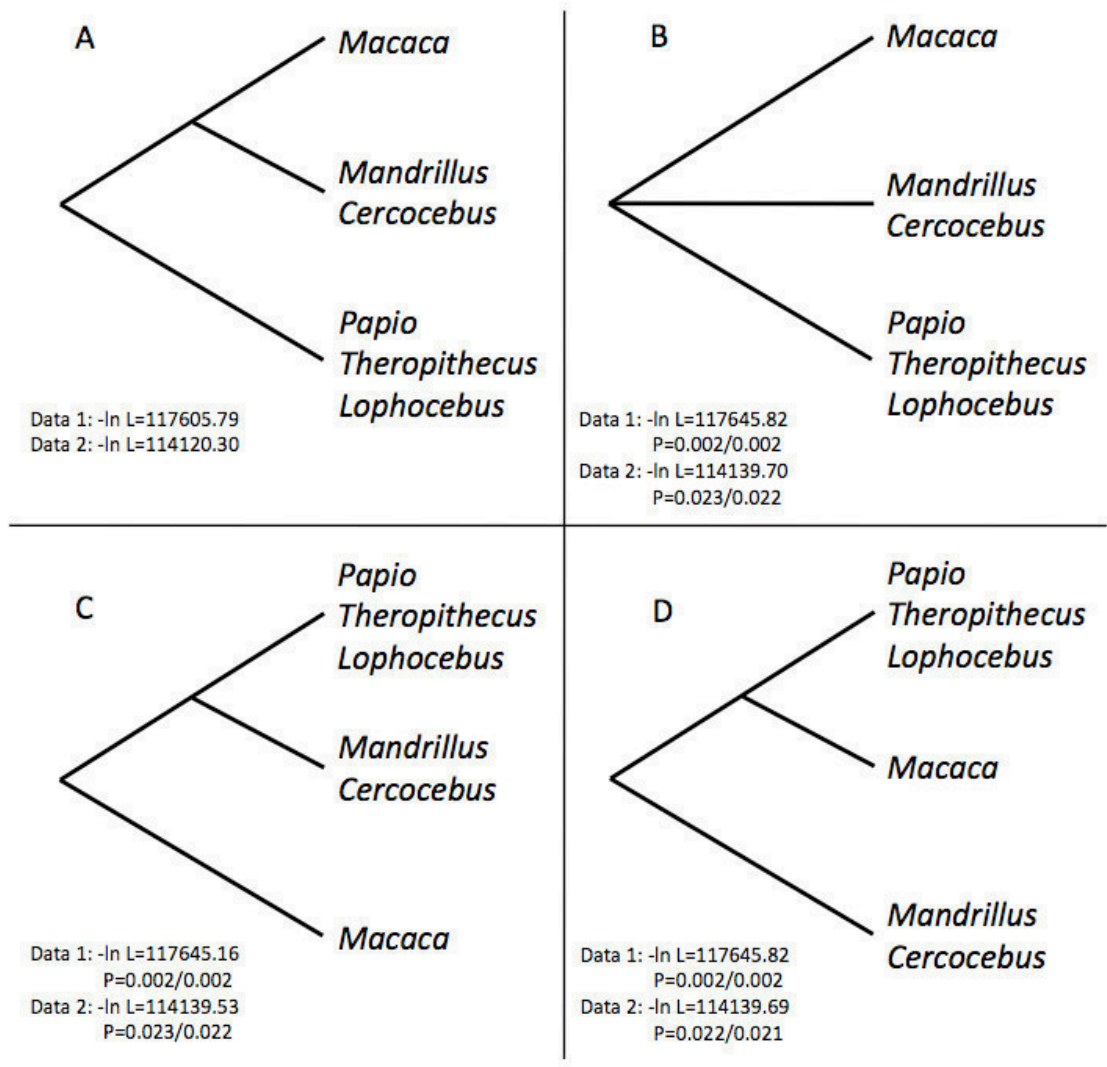

Figure 3 Tree topologies that were tested in the alternative tree topology test. Tree A represents the most probable topology, whereas $\mathbf{B}, \mathbf{C}$ and $\mathbf{D}$ were significantly rejected. Log-likelihood and $\mathbf{P}$ values for each tree topology are given for dataset 1 and 2, respectively. First and second $\mathrm{P}$ values resulted from the KishinoHasegawa and the Shimodaira-Hasegawa tests, respectively.

Our mtDNA genome tree revealed paraphyletic relationships of Mandrillus and Cercocebus taxa, which is again contradictory to nDNA studies that suggest both genera to be reciprocally monophyletic [14,15]. As our data show, M. leucophaeus clusters with $C$. chrysogaster and M. sphinx is indicated as sister lineage to both to the exclusion of $C$. torquatus and $C$. atys. Again, ancient hybridization and incomplete lineage sorting cannot be excluded as having affected this branching pattern. However, since the species identification of the herein used $C$. torquatus sample is questionable (originally identified as Lophocebus albigena [78]), our results have to be regarded as preliminary and at the moment any further discussion of possible phylogeographic scenarios would remain highly speculative. Interestingly, however, the sister relationship of $C$. chrysogaster to $M$. leucophaeus is consistent with Kingdon's [79] p.46 observation that $C$. chrysogaster is morphologically "the 
most drill-like of the drill-mangabeys". On the other hand, Kingdon's suggestion has not been held up by several other studies, which find $C$. torquatus to be the most primitive and Mandrillus-like mangabey [14,45,46,55,72]. Comprehensive sampling of mangabeys with reliable information on their geographic provenance is required to further elucidate relationships within the Mandrillus + Cercocebus clade.

Relationships among Papio, Theropithecus and Lophocebus have been analysed in several studies, but differed depending on the markers that were applied. Chatterjee at al. [56] investigated seven mitochondrial genes and found Theropithecus clustering with Lophocebus to the exclusion of Papio while Finstermeier et al. [19] showed a closer, but only weakly supported mtDNA genome affiliation of Papio to Theropithecus; Pozzi et al. [20] were also not able to resolve these relationships. Likewise, while we found Theropithecus split off first in the Bayesian analysis of the original dataset, ML analysis as well as both, Bayesian and ML estimations of dataset 2 suggested Lophocebus in the basal position. For both datasets, support values for respective branching patterns are low and estimated divergence ages among the three genera indicate a rapid radiation around $5 \mathrm{Ma}$. Also in the Densitree, different branching patterns are depicted. Accordingly, the present data are probably not sufficient to resolve the branching pattern. On the other hand, nDNA sequence data revealed a more consistent picture by placing Lophocebus with Papio to the exclusion of Theropithecus [14,15,48,56,71]. Not surprisingly, morphological (i.e., craniodental) data are congruent with these molecular studies when allometry is properly accounted [80,81]. Guevara \& Steiper [14] stated that the basal position of Theropithecus is plausible given that known fossils [82] of the genus are considerably older $(\sim 4.0 \mathrm{Ma})$ than that of Papio $(\sim 2.5 \mathrm{Ma})$ and Lophocebus ( 2.0 Ma). It has been shown that an increased sampling of more individuals per species may help to resolve phylogenies with short internodes, but nevertheless an increased sampling will not improve the phylogenies when hybridisation has confounded it [14,74].

The initial radiation within the Papionini into the three main lineages 1) Papio, Theropithecus and Lophocebus, 2) Mandrillus and Cercocebus, and 3) Macaca took place during the Late Miocene. Within these three clades, further differentiation events occurred on similar time scales (Theropithecus - Lophocebus - Papio: 5-6 Ma; Mandrillus - Cercocebus: 4-5 Ma; Macaca: 5-6 Ma). (Figure 1, Additional file 2: 
Table S1, Additional file 4: Figure S2). This means that, although macaques seem morphologically not as diverse as their African sister taxa [23,35,83], the mitochondrial heterogeneity among species groups is at least as high as among the African papionin genera. Comparing our mtDNA divergence ages with those inferred from nDNA data (e.g. [15]) we find that those splits slightly differ but tend to be in the same range (Additional file 2: Table S1). We therefore can assume nuclear heterogeneity among Macaca species groups and Papionina genera to be also similar.

Given the equally long independent evolutionary histories of macaque species groups and Papionina genera the question of whether the species groups represent rather distinct genera or whether the two main African Papionina clades constitute only two genera (Papio and Cercocebus) with diverse species groups seems a subject for debate. However, due to morphological similarities of the macaque taxa and the morphological differences between the African genera, a reorganisation of their taxonomic ranks based on time depths as proposed by Goodman [84] and Groves $[23,85]$ seems not to be justified at the moment.

\section{Conclusion}

By analysing complete mtDNA genomes of all papionin genera (with the exception of Rungwecebus) we obtained well-resolved phylogenetic relationships and higher support values than inferred from shorter mtDNA fragments. Our estimated divergence ages are similar to those of other studies but credibility intervals are narrowed down due to the application of complete mtDNA genome sequences. Including an increased number of papionin samples led to a different tree topology concerning the phylogenetic position of the Mandrillus + Cercocebus clade among papionins, which is in stark contrast to previous nDNA studies, indicating that ancient introgression or incomplete lineage sorting may play a role here. However, which of the two processes led to these contradictions cannot be determined here since we analysed only the maternal lineage of included taxa.

Although the mtDNA tree is just a single gene tree, it offers important additional information on the evolutionary history of the Papionini. Future investigations should incorporate a large number of nDNA loci or even complete genome data to possibly 
distinguish introgression or incomplete lineage sorting. Furthermore, for a reliable comparative study of mtDNA and nDNA sequences data, respective loci are at best obtained from the same individuals or at least the same species. Since respective nDNA data is by now not available from GenBank we focused solely on mtDNA data. In addition to nDNA data future studies should also include comprehensive sequence data of the herein unstudied genus Rungwecebus. There is also a need to further elucidate intra-generic taxonomy and phylogeny in almost all papionin genera, particularly in Cercocebus. Therefore special attention must be paid to the geographic provenance of studied samples.

\section{Methods}

\section{Sample collection}

Blood samples from one individual each of $M$. arctoides ( $M$. arctoides group), $M$. silenus ( $M$. silenus group), $M$. tonkeana (Sulawesi group), $M$. fascicularis ( $M$. fascicularis group) and $M$. sylvanus (M. sylvanus group), and two individuals of $M$. mulatta ( $M$. mulatta group) were obtained from European zoos, Covance and the German Primate Center. All blood samples were taken during routine health checks by experienced veterinarians and not specifically for this study. A fresh tissue sample from a deceased $M$. thibetana ( $M$. sinica group) individual was obtained from the Strasbourg Primate Center. Sample collection was approved by the Animal Welfare Body of the German Primate Center and adhered to the American Society of Primatologists Principles for the Ethical Treatment of Non-Human Primates (see www.asp.org/ society/policy.cfm). No animals were sacrificed for this study.

\section{Laboratory methods}

Genomic DNA from blood and tissue samples was extracted using the Qiagen DNeasy Blood \& Tissue Kit following the supplier's recommendations. To minimize the chance of amplifying nuclear mitochondrial-like sequences (numts) [86], two overlapping long-range PCR fragments were generated (8 kb and $10 \mathrm{~kb}$ ) using primers specifically designed for macaque species groups on the basis of available sequence data in GenBank and the Long Range dNTPack from Roche. Conditions for the long-range PCR amplification comprised a pre-denaturation step at $94^{\circ} \mathrm{C}$ for 2 
min, followed by 40 cycles at $94^{\circ} \mathrm{C}$ for $1 \mathrm{~min}$, annealing at $60^{\circ} \mathrm{C}$ for $1 \mathrm{~min}$ and extension at $68^{\circ} \mathrm{C}$ for $20 \mathrm{~min}$. At the end a final extension step at $68^{\circ} \mathrm{C}$ for $30 \mathrm{~min}$ was added. PCR products were visualized on $1 \%$ agarose gel and extracted with the Qiagen PCR purification Kit. Obtained long-range fragments were used as template for nested PCRs to generate products of 1.0 to $1.2 \mathrm{~kb}$. Respective primers are available from the authors upon request. PCR conditions for nested PCRs comprised a pre-denaturation step at $94^{\circ} \mathrm{C}$ for $2 \mathrm{~min}$, followed by 40 cycles each with denaturation at $94^{\circ} \mathrm{C}$ for $1 \mathrm{~min}$, annealing at $60^{\circ} \mathrm{C}$ for $1 \mathrm{~min}$ and extension at $72^{\circ} \mathrm{C}$ for $1.5 \mathrm{~min}$, and terminating with a final extension step at $72^{\circ} \mathrm{C}$ for $5 \mathrm{~min}$. PCR products were again checked on $1 \%$ agarose gels, and subsequently extracted and sequenced on an $A B I 3130 x L$ sequencer using the BigDye Terminator Cycle Sequencing Kit (Applied Biosystems) and the amplification primers. DNA extraction, PCR set-up, gel extraction and sequencing were performed in separate laboratories. Genome sequences were assembled with SeaView 4.4.0. [87] and annotation was conducted with the online program DOGMA [88] and manually checked. Sequences in the overlapping parts of the two long-range PCRs were identical and all proteincoding genes were correctly translated without any premature stop codons, indicating that no numt contamination is present in our data. All sequences were deposited at GenBank (for accession numbers see Additional file 7: Table S2).

\section{Data analysis}

The dataset for the phylogenetic analysis comprised a total of $38 \mathrm{mtDNA}$ genome sequences including 13 macaques representing all seven species groups (2 M. sylvanus, $1 \mathrm{M}$. silenus, $1 \mathrm{M}$. tonkeana, $2 \mathrm{M}$. thibetana, $3 \mathrm{M}$. mulatta, $3 \mathrm{M}$. fascicularis and $1 M$. arctoides), eleven baboons (2 P. ursinus, 2 P. hamadryas, $3 P$. anubis, $2 P$. cynocephalus, 1 P. kindae and 1 P. papio), three geladas (T. gelada), one drill ( $M$. leucophaeus), one mandrill (M. sphinx), one crested mangabey (L. aterrimus), three capped mangabeys $(1 \mathrm{C}$. chrysogaster, 1 C. atys, 1 C. torquatus) and five nonpapionin primate species (Chlorocebus pygerythrus, Colobus guereza, Pongo abelii, Pan troglodytes, Homo sapiens). Accordingly, Rungwecebus was the only missing papionin genus. The identity of the $C$. torquatus individual remained ambiguous. While it was originally assigned to Lophocebus albigena [78], BLAST-search revealed that it is $99-100 \%$ identical to available mtDNA sequences of $C$. torquatus. 
For information about GenBank accession numbers and the source of the herein used sequences see Additional file 7: Table S2.

Sequences were aligned with Muscle 3.7 [89] as implemented in SeaView and manually corrected. For phylogenetic tree reconstructions, indels and poorly aligned positions were removed with Gblocks $0.91 \mathrm{~b}$ [90]. To check for possible shifts in base composition among species, we calculated the base composition for each species using PAUP 4.0b10 [91]. Since we observed a slight shift in A/C content among papionins (Additional file 3: Figure S1) and to test whether this shift might have influenced phylogenetic relationships, we generated a second alignment (dataset 2) in which positions that contained both an Adenin and Cytosin were masked with an "M" (in total 606 positions).

The programs RAxML 0.93 [92] and MrBayes 3.1 .2 [93,94] were used for phylogenetic tree reconstructions applying $\mathrm{ML}$ and Bayesian algorithms. As substitution models for Bayesian reconstructions we applied the $\operatorname{TrN}+\mathrm{I}+\mathrm{G}$ and GTR $+I+G$ models for datasets 1 and 2, respectively, as they were selected as best-fit models by jModeltest 2.1 [95] under the Bayesian information criterion (BIC) and the Decision Theory Performance-based Selection (DT). In MrBayes we analysed four independent Markov Chain Monte Carlo (MCMC) runs with a default temperature of 0.2. All repetitions were run for 1 million generations with tree and parameter sampling setting in every 100 generations. The first $25 \%$ of samples were discarded as burn-in, resulting in 75,001 trees per run. The adequacy of the burn-in and convergence of all parameters was assessed via the uncorrected potential scale reduction factor (PSRF) [96] as calculated by MrBayes and by visual inspection of the trace of the parameters across generations using the software TRACER 1.5 [97]. To check whether posterior clade probabilities were also converging, AWTY [98] was used. Posterior probabilities for each split and a phylogram with mean branch lengths were calculated from the posterior density of trees. Both $M L$ calculations in RAXML were run with the CAT-GTR model and 1,000 rapid bootstrapping replications. Alternative phylogenetic relationships among the three observed major papionin clades were tested with the Kishino-Hasegawa test [99] and ShimodairaHasegawa test [100] with full optimisation and 1,000 bootstrap replications in PAUP. 
Divergence ages were estimated applying both, uncorrelated and auto-correlated, clock models. To calculate divergence ages with an uncorrelated clock model, we used BEAST 1.6.1 [101,102]. We assumed a relaxed lognormal model of lineage variation and a Birth-Death Process prior for branching rates. In contrast to Finstermeier et al. [19], branching of Mandrillus + Cercocebus with Macaca was not constrained in our study as alternative branching patterns were rejected by alternative tree topology tests.

The following five fossil-based calibration points were applied with a normal distribution prior for respective nodes: The Homo - Pan split 6.5 Ma with a 95\% credibility interval $(\mathrm{Cl})$ of $0.5 \mathrm{Ma}$ [103-105]. The split between Pongo and the HomoPan lineage at $14.0 \mathrm{Ma}(95 \% \mathrm{Cl}: 1.0 \mathrm{Ma})$ [106], the divergence of Theropithecus and Papio $5.0 \mathrm{Ma}(95 \% \mathrm{Cl}$ : $1.5 \mathrm{Ma})$ [107,108], the split between African and Asian macaques at $5.5 \mathrm{Ma}(95 \% \mathrm{Cl}: 1.0 \mathrm{Ma})[27,108]$ and the separation of hominoids and cercopithecoids at $27.5 \mathrm{Ma}(95 \% \mathrm{Cl}: 3.5 \mathrm{Ma})$ [109-111].

In total, we ran four replicates in BEAST, each with 25 million generations, and tree and parameter sampling every 1,000 generations. TRACER was applied to assess the adequacy of a $10 \%$ burn-in and the convergence. The sampling distributions were combined (25\% burn-in) with LogCombiner 1.6.1 and a consensus chronogram with node height distribution was generated and visualized with TreeAnnotator 1.6.1 and FigTree 1.4.0 [112].

To see whether the application of an auto-correlated model instead of an uncorrelated model has an effect on the divergence time estimation we performed Bayesian molecular dating with the software package PhyloBayes 3.3 [113]. The tree topology was fixed using the topology as inferred from MrBayes. Five node ages were fixed by specifying calibration intervals based on the same calibration points and credibility interval as mention above. In the main program of PhyloBayes ( $p b$ ) the CAT-GTR model was applied in combination with a log-normal auto-correlated (-In) [114] relaxed clock model and in a second independent run with an uncorrelated (-ugam) [101] relaxed clock model. We monitored the development of the log-likelihood as a function of time and found it to be stable (to show convergence) after approximately 3,000-4,000 cycles. Hence, 10,000 cycles were carried out discarding the first 2,500 trees as burn-in. A posterior consensus 
chronogram was calculated on the remaining 7,500 trees using the post analysis program readpb and was visualized with Figtree.

\section{Availability of supporting data}

The data set supporting the results of this article is available in the Data Dryad repository, DOI: 10.5061/dryad.9tm42

\section{Competing interests}

The authors declare that they have no competing interests.

\section{Authors' contributions}

$\mathrm{RL}$ did laboratory work, analysed data, and wrote the paper. MB analysed data. DZ and CR designed the study, analysed data, and wrote the paper. All authors read and approved the final manuscript.

\section{Acknowledgements}

We thank the zoos in Dresden, Madrid, Salem, Straubing and Wuppertal as well as Covance Inc. (Münster, Gemany), the Strasbourg Primate Center and the German Primate Center for providing valuable macaque samples. We are also grateful to Christiane Schwarz for her excellent laboratory work, and Colin Groves and Brandon C. Wheeler for their valuable comments on an earlier version of the paper and their corrections of the English. We thank the editor and three anonymous reviewers for critical comments on an earlier version of the manuscript. 


\section{References}

1. Moore WS: Inferring phylogenies from mtDNA variation: mitochondrial-gene trees versus nuclear-gene trees. Evolution 1995, 49:718-726.

2. Hoelzer GA: Inferring phylogenies from mtDNA variation: Mitochondrial-gene trees versus nuclear-gene trees revisited. Evolution 1997, 51:622-626.

3. Avise JC: Molecular Markers, Natural History, and Evolution. Sunderland, MA: Sinauer Associates; 2004.

4. Maddison WP: Gene trees in species trees. Syst Biol 1997, 46:523-536.

5. Funk DJ, Omland KE: Species-level paraphyly and polyphyly: frequency, causes, and consequences, with insights from animal mitochondrial DNA. Ann Rev Ecol Evol Syst 2003, 34:397-423.

6. Avise JC: Phylogeography: retrospect and prospect. J Biogeograph 2009, 36:315.

7. Tosi AJ, Morales JC, Melnick DJ: Y-chromosome and mitochondrial markers in Macaca fascicularis indicate introgression with Indochinese $\mathrm{M}$. mulatta and a biogeographic barrier in the Isthmus of Kra. Int J Primatol 2002, 23:161-178.

8. Storz JF, Ramakrishnan U, Alberts SC: Genetic effective size of a wild primate population: influence of current and historical demography. Evolution 2002, 56:817829.

9. Roberts TE, Davenport TRB, Hildebrandt KBP, Jones T, Stanley WT, Sargis EJ, Olson LE: The biogeography of introgression in the critically endangered African monkey Rungwecebus kipunji. Biol Lett 2010, 6:233-237.

10. Zinner D, Arnold ML, Roos C: Is the new primate genus Rungwecebus a baboon? PLoS One 2009, 4:e4859.

11. Zinner D, Groeneveld LF, Keller C, Roos C: Mitochondrial phylogeography of baboons (Papio spp.) - Indication for introgressive hybridization? BMC Evol Biol 2009, 9:83.

12. Zinner D, Arnold ML, Roos C: The strange blood: Natural hybridization in primates. Evol Anthropol 2011, 20:96-103.

13. Satkoski Trask JA, Garnica WT, Smith DG, Houghton P, Lerche N, Kanthaswamy S: Single-Nucleotide Polymorphisms reveal patterns of allele sharing across the species boundary between rhesus (Macaca mulatta) and cynomolgus ( $M$. fascicularis) macaques. Am J Primatol 2013, 75:135-144.

14. Guevara EE, Steiper ME: Molecular phylogenetic analysis of the Papionina using concatenation and species tree methods. J Human Evol 2014, 66:18-28. 
15. Perelman $P$, Johnson WE, Roos $C$, Seuánez HN, Horvath JE, Moreira MAM, Kessing B, Pontius J, Roelke M, Rumpler Y, Schneider MPC, Silva A, O'Brien SJ, Pecon-Slattery J: A molecular phylogeny of living primates. PLoS Genet 2011, 7:e1001342.

16. Zinner D, Fickenscher G, Roos C: Family Cercopithecidae (Old World Monkeys). In The Handbook of the Mammals of the World, Primates, Volume 3. Edited by Mittermeier RA, Rylands AB, Wilson DE. Barcelona: Lynx Edicions; 2013:550-627.

17. Page SL, Goodman M: Catarrhine phylogeny: noncoding DNA evidence for a diphyletic origin of the mangabeys and for a human-chimpanzee clade. Mol Phylogenet Evol 2001, 18:14-25.

18. Xing J, Wang H, Han K, Ray DA, Huang CH, Chemnick LG, Stewart CB, Disotell TR, Ryder OA, Batzer MA: A mobile element based phylogeny of Old World monkeys. Mol Phylogenet Evol 2005, 37:872-880.

19. Finstermeier K, Zinner D, Brameier M, Meyer M, Kreuz E, Hofreiter M, Roos C: A mitogenomic phylogeny of living primates. PLoS One 2013, 8:e69504.

20. Pozzi L, Hodgson JA, Burrell AS, Sterner KN, Raaum RL, Disotell TR: Primate phylogenetic relationships and divergence dates inferred from complete mitochondrial genomes. Mol Phylogenet Evol. in press. http://dx.doi.org/10.1016/j.ympev.2014.02.023.

21. Delson E: Evolutionary history of the Cercopithecidae. Contrib Primatol 1975, 5:167-217.

22. Stewart CB, Disotell TR: Primate evolution - in and out of Africa. Curr Biol 1998, 8:R582-R588.

23. Groves CP: Primate Taxonomy. Washington, DC: Smithsonian Institution Press; 2001.

24. Böhm M, Mayhew P: Historical biogeography and the evolution of the latitudinal gradient of species richness in the Papionini (Primata: Cercopithecidae). Biol J Linnean Soc 2005, 85:235-246.

25. Jolly CJ: Tribe Papionini. In Mammals of Africa, Primates, Volume II. Edited by Butynski TM, Kingdon J, Kalina J. London: Bloomsbury Publishing; 2013:157-158.

26. Delson E: Fossil macaques, phyletic relationships and a scenario of deployment. In The Macaques: Studies in Ecology, Behavior and Evolution. Edited by Lindhurg D. New York: Van Nostrand Reinhold; 1980:10-30.

27. Alba DM, Delson E, Carnevale G, Colombero S, Delfino M, Giuntelli P, Pavia M, Pavia G: First joint record of Mesopithecus and cf. Macaca in the Miocene of Europe. J Hum Evol 2014, 67:1-18.

28. Gupta VJ, Sahni A: Theropithecus delsoni, a new cercopithecine species from the Upper Siwaliks of India. Bull Ind Geol Ass 1981, 14:69-71. 
29. Delson E: Theropithecus fossils from Africa and India and the taxonomy of the genus. In Theropithecus: The Rise and Fall of a Primate Genus. Edited by Jablonski NG. Cambridge: Cambridge University Press; 1993:157-189.

30. Delson E, Eck GG, Leakey MG, Jablonski NG: A partial catalogue of fossil remains of Theropithecus. In Theropithecus: The Rise and Fall of a Primate Genus. Edited by Jablonski NG. Cambridge: Cambridge University Press; 1993:499-525.

31. Pickford $\mathrm{M}$ : Climatic change, biogeography, and Theropithecus. In Theropithecus: The Rise and Fall of a Primate Genus. Edited by Jablonski NG. Cambridge: Cambridge University Press; 1993:227-243.

32. Gibert J, Ribot F, Gibert L, Leakey M, Arribas A, Martinez B: Presence of the cercopithecid genus Theropithecus in Cueva Victoria (Murcia, Spain). J Hum Evol 1995, 28:487-493.

33. Rook L, Martínez-Navarro B, Clark Howell F: Occurrence of Theropithecus sp. in the Late Villafranchian of Southern Italy and implication for Early Pleistocene "out of Africa" dispersals. J Hum Evol 2004, 47:267-277.

34. Roberts $P$, Delson E, Miracle P, Ditchfield P, Roberts RG, Jacobs Z, Blinkhorn J, Ciochon RL, Fleagle JG, Frost SR, Gilbert CC, Gunnell GF, Harrison T, Korisettar R, Petraglia MD: Continuity of mammalian fauna over the last 200,000 y in the Indian subcontinent. Proc Natl Acad Sci U S A 2014, 111(16):5848-5853.

35. Fooden J: Provisional classification and key to living species of macaques (Primates: Macaca). Folia Primatol 1976, 25:225-236.

36. Anandam MV, Bennett EL, Davenport TRB, Davies NJ, Detwiler KM, Engelhardt A, Eudey AA, Gadsby EL, Groves CP, Healy A, Karanth KP, Molur S, Nadler T, Richardson MC, Riley EP, Roos C, Rylands AB, Sheeran LK, Ting N, Wallis J, Waters SS, Whittaker DJ, Zinner D: Species accounts of Cercopithecidae. In The Handbook of the Mammals of the World, Primates, Volume 3. Edited by Mittermeier RA, Rylands AB, Wilson DE. Barcelona: Lynx Edicions; 2013:628-753.

37. Tosi AJ, Morales JC, Melnick DJ: Paternal, maternal, and biparental molecular markers provide unique windows onto the evolutionary history of macaque monkeys. Evolution 2003, 57:1419-1435.

38. Ziegler T, Abegg C, Meijaard E, Perwitasari-Farajallah D, Walter L, Hodges JK, Roos C: Molecular phylogeny and evolutionary history of Southeast Asian macaques forming the M. silenus group. Mol Phylogenet Evol 2007, 42:807-816.

39. Hayasaka K, Fujii K, Horai S: Molecular phylogeny of macaques: implications of nucleotide sequences from an 896 base pair region of mitochondrial DNA. Mol Biol Evol 1996, 13:1044-1053.

40. Morales JC, Melnick DJ: Phylogenetic relationships of the macaques (Cercopithecidae: Macaca), as revealed by high resolution restriction site mapping of mitochondrial ribosomal genes. J Hum Evol 1998, 34:1-23. 
41. Disotell TR, Honeycutt RL, Ruvolo M: Mitochondrial DNA phylogeny of the OldWorld monkey tribe Papionini. Mol Biol Evol 1992, 9:1-13.

42. Szalay FS, Delson E: Evolutionary History of the Primates. New York: Academic; 1979.

43. Strasser E, Delson E: Cladistic analysis of cercopithecid relationships. J Hum Evol 1987, 16:81-99.

44. Groves CP: Phylogenetic and population systematics of the mangabeys (Primates: Cercopithecoidea). Primates 1978, 19:1-34.

45. Fleagle JG, McGraw WS: Skeletal and dental morphology supports diphyletic origin of baboons and mandrills. Proc Natl Acad Sci U S A 1999, 96:1157-1161.

46. Gilbert CC: Craniomandibular morphology supporting the diphyletic origin of mangabeys and a new genus of the Cercocebus/Mandrillus clade, Procercocebus. J Hum Evol 2007, 53:69-102.

47. Harris EE, Disotell TR: Nuclear gene trees and the phylogenetic relationships of the mangabeys (Primates: Papionini). Mol Biol Evol 1998, 15:892-900.

48. Harris EE: Molecular systematics of the Old World monkey tribe Papionini: analysis of the total available genetic sequences. J Hum Evol 2000, 38:235-256.

49. Jones T, Ehardt CL, Butynski TM, Davenport TRB, Mpunga NE, Machaga SJ, De Luca DW: The highland mangabey Lophocebus kipunji: a new species of African monkey. Science 2005, 308:1161-1164.

50. Davenport TRB, Stanley WT, Sargis EJ, De Luca DW, Mpunga NE, Machaga SJ, Olson LE: A new genus of African monkey, Rungwecebus: morphology, ecology, and molecular phylogenetics. Science 2006, 312:1378-1381.

51. Burrell AS, Jolly CJ, Tosi AJ, Disotell TR: Mitochondrial evidence for the hybrid origin of the kipunji, Rungwecebus kipunji (Primates: Papionini). Mol Phylogenet Evol 2009, 51:340-348.

52. Newman TK, Jolly CJ, Rogers J: Mitochondrial phylogeny and systematics of baboons (Papio). Am J Phys Anthropol 2004, 124:17-27.

53. Wildman DE, Bergman TJ, al-Aghbari A, Sterner KN, Newman TK, PhillipsConroy JE, Jolly CJ, Disotell TR: Mitochondrial evidence for the origin of hamadryas baboons. Mol Phylogenet Evol 2004, 32:287-296.

54. Zinner D, Wertheimer J, Liedigk R, Groeneveld LF, Roos C: Baboon phylogeny as inferred from complete mitochondrial genomes. Am J Phys Anthropol 2013, 150:133-140.

55. Fleagle JG, McGraw WS: Skeletal and dental morphology of African papionins: unmasking a cryptic clade. J Hum Evol 2002, 42:267-292. 
56. Chatterjee HJ, Ho SYW, Barnes I, Groves C: Estimating the phylogeny and divergence times of primates using a supermatrix approach. BMC Evol Biol 2009, 9:259.

57. Chan YC, Roos C, Inoue-Murayama M, Inoue E, Shih CC, Pei KJC, Vigilant L: Mitochondrial genome sequences effectively reveal the phylogeny of Hylobates gibbons. PLoS One 2010, 5:e14419.

58. Chiou KL, Pozzi L, Lynch Alfaro JW, Di Fiore A: Pleistocene diversification of living squirrel monkeys (Saimiri spp.) inferred from complete mitochondrial genome sequences. Mol Phylogenet Evol 2011, 59:736-745.

59. Roos C, Zinner D, Kubatko L, Schwarz C, Yang M, Meyer D, Nash S, Xing J, Batzer MA, Brameier M, Leendertz FH, Ziegler T, Perwitasari-Farjajjah D, Nadler T, Walter L, Osterholz M: Nuclear versus mitochondrial DNA: evidence for hybridization in colobine monkeys. BMC Evol Biol 2011, 11:77.

60. Liedigk R, Yang M, Jablonski NG, Momberg F, Geissmann T, Lwin N, Hla TH, Liu Z, Wong B, Ming L, Yongcheng L, Zhang YP, Nadler T, Zinner D, Roos C: Evolutionary history of the odd-nosed monkeys and the phylogenetic position of the newly described Myanmar snub-nosed monkey Rhinopithecus strykeri. PLoS One 2012, 7:e37418.

61. Supplementary data from: Mitogenomics of the Old World monkey tribe Papionini. Dryad Digital Repository. [http://doi:10.5061/dryad.9tm42].

62. Bouckaert RR: DensiTree: making sense of sets of phylogenetic trees. Bioinformatics 2010, 26:1372e1373.

63. Pollard DA, lyer VN, Moses AM, Eisen MB: Widespread discordance of gene trees with species tree in Drosophila: evidence for incomplete lineage sorting. PLoS Genet 2006, 2:e173.

64. Raaum RL, Sterner KN, Noviello CM, Stewart CB, Disotell TR: Catarrhine primate divergence dates estimated from complete mitochondrial genomes: concordance with fossil and nuclear DNA evidence. J Hum Evol 2005, 48:237-257.

65. Steiper ME, Young NM: Primate molecular divergence dates. Mol Phylogenet Evol 2006, 41:384-394.

66. Li J, Han K, Xing J, Kim HS, Rogers J, Ryder OA, Disotell T, Yue B, Batzer MA: Phylogeny of macaques (Cercopithecidae: Macaca) based on Alu elements. Gene 2009, 448:242-249.

67. Pollock DD, Zwickl DJ, Mcguire JA, Hillis DM: Increased taxon sampling is advantageous for phylogenetic inference. Syst Biol 2002, 51:664-671.

68. Zwickl DJ, Hillis DM: Increased taxon sampling greatly reduces phylogenetic error. Syst Biol 2002, 51:588-598. 
69. Townsend JP, Leuenberger C: Taxon sampling and the optimal rates of evolution for phylogenetic inference. Syst Biol 2011, 60:358-365.

70. Nabhan AR, Sarkar IN: The impact of taxon sampling on phylogenetic inference: a review of two decades of controversy. Brief Bioinform 2012, 13:122-134.

71. Springer MS, Meredith RW, Gatesy J, Emerling CA, Park J, Rabosky DL, Stadler T, Steiner C, Ryder OA, Janecka JE, Fisher CA, Murphy WJ: Macroevolutionary dynamics and historical biogeography of primate diversification inferred from a species supermatrix. PLoS One 2012, 7:e49521.

72. McGraw WS, Fleagle JG: Biogeography and evolution of the CercocebusMandrillus clade: evidence from the face. In Primate Biogeography: Progress and Prospects. Edited by Lehman SM, Fleagle JG. New York: Springer; 2006:201-224.

73. Ito $T$, Nishimura $T$, Takai $M$ : Ecogeographical and phylogenetic effects on craniofacial variation in macaques. Am J Phys Anthropol 2014, in press.

74. Maddison WP, Knowles LL: Inferring phylogeny despite incomplete lineage sorting. Syst Biol 2006, 55:21-30.

75. Keller C, Roos C, Groeneveld LF, Fischer J, Zinner D: Introgressive hybridization in southern African baboons shapes patterns of mtDNA variation. Am J Phys Anthropol 2010, 142:125-136.

76. Arnold ML, Meyer A: Natural hybridization in primates: One evolutionary mechanism. Zoology 2006, 109:261-276.

77. Chiarelli B: Check-list of catarrhina primate hybrids. J Hum Evol 1973, 2:301305.

78. Guschanski K, Krause J, Sawyer S, Valente LM, Bailey S, Finstermeier K, Sabin R, Gilissen E, Sonet G, Nagy ZT, Lenglet G, Mayer F, Savolainen V: Nextgeneration museomics disentangles one of the largest primate radiations. Syst Biol 2013, 62:539-554.

79. Kingdon J: The Kingdon Field Guide to African Mammals. London: Academic; 1997.

80. Gilbert CC, Rossie JB: Congruence of molecules and morphology using a narrow allometric approach. Proc Natl Acad Sci U S A 2007, 104:11910-11914.

81. Gilbert CC: African papionin phylogenetic history and Plio-Pleistocene biogeography, PhD thesis. Stony Brook University; 2008.

82. Gilbert CC: Cladistic analysis of extant and fossil African papionins using craniodental data. J Hum Evol 2013, 64:399-433.

83. Fooden J: Classification and distribution of living macaques. In The Macaques: Studies in Ecology, Behavior, and Evolution. Edited by Lindburg DG. New York: Van Nostrand-Reinhold; 1980:1-9. 
84. Goodman M, Porter CA, Czelusniak J, Page SL, Schneider H, Shoshani J, Gunnell G, Groves CP: Toward a phylogenetic classification of primates based on DNA evidence complemented by fossil evidence. Mol Phylogenet Evol 1998, 9:585598.

85. Groves CP: The what, why and how of primate taxonomy. Int J Primatol 2004, 25:1105-1126.

86. Thalmann O, Hebler J, Poinar HN, Pääbo S, Vigilant L: Unreliable mtDNA data due to nuclear insertions: a cautionary tale from analysis of humans and other great apes. Mol Ecol 2004, 13:321-325.

87. Gouy M, Guindon S, Gascuel O: SeaView version 4: a multiplatform graphical user interface for sequence alignment and phylogenetic tree building. Mol Biol Evol 2010, 27:221-224.

88. Wyman SK, Jansen RK, Boore JL: Automatic annotation of organellar genomes with DOGMA. Bioinformatics 2004, 20:3252-3255.

89. Edgar RC: MUSCLE: multiple sequence alignment with high accuracy and high throughput. Nucleic Acids Res 2004, 32:1792-1797.

90. Castresana J: Selection of conserved blocks from multiple alignments for their use in phylogenetic analysis. Mol Biol Evol 2000, 17:540-552.

91. Swofford DL: PAUP* Phylogenetic Analysis using Parsimony ( ${ }^{*}$ and other Methods), Version 4. Sunderland: Sinauer Associates; 2003.

92. Stamatakis A: RAxML-VI-HPC: maximum likelihood-based phylogenetic analyses with thousands of taxa and mixed models. Bioinformatics 2006, 22:26882690.

93. Huelsenbeck JP, Ronquist F, Nielsen R, Bollback JP: Bayesian inference of phylogeny and its impact on evolutionary biology. Science 2001, 294:2310-2314.

94. Ronquist F, Huelsenbeck JP: MrBayes 3: Bayesian phylogenetic inference under mixed models. Bioinformatics 2003, 19:1572-1574.

95. Posada D: Selection of models of DNA evolution with jModelTest. Methods Mol Biol 2009, 537:93-112.

96. Gelman A, Rubin D: Inference from iterative simulation using multiple sequences. Stat Sci 1992, 7:457-511.

97. Rambaut A, Drummond AJ: Tracer: MCMC trace analysis tool, version 1.5. [http://tree.bio.ed.ac.uk/sofware/tracer/].

98. Nylander JA, Wilgenbusch JC, Warren DL, Swofford DL: AWTY (are we there yet?): a system for graphical exploration of MCMC convergence in Bayesian phylogenetics. Bioinformatics 2008, 24:581-583. 
99. Kishino $\mathrm{H}$, Hasegawa $\mathrm{M}$ : Evaluation of the maximum likelihood estimate of the evolutionary tree topologies from DNA sequence data, and their branching order of Hominoidea. J Mol Evol 1989, 29:170-179.

100. Shimodaira H, Hasegawa M: Multiple comparisons of log likelihoods with applications to phylogenetic inference. Mol Biol Evol 1999, 16:1114-1116.

101. Drummond AJ, Ho SY, Phillips MJ, Rambaut A: Relaxed phylogenetics and dating with confidence. PLoS Biol 2006, 4:e88.

102. Drummond AJ, Rambaut A: BEAST: Bayesian evolutionary analysis by sampling trees. BMC Evol Biol 2007, 7:214.

103. Vignaud $P$, Duringer $P$, Mackaye HT, Likius A, Blondel $C$, Boisserie JR, De Bonis L, Eisenmann V, Etienne ME, Geraads D, Guy F, Lehmann T, Lihoreau F, Lopez-Martinez N, Mourer-Chauvire C, Otero O, Rage JC, Schuster M, Viriot L, Zazzo A, Brunet M: Geology and palaeontology of the upper Miocene Toros-Menalla hominid locality, Chad. Nature 2002, 418:152-155.

104. Brunet M, Guy F, Pilbeam D, Lieberman DE, Likius A, Mackaye HT, Ponce de León MS, Zollikofer CP, Vignaud P: New material of the earliest hominid from the upper Miocene of Chad. Nature 2005, 434:752-755.

105. Lebatard AE, Bourles DL, Duringer $P$, Jolivet $M$, Braucher $R$, Carcaillet J, Schuster M, Arnaud N, Monie P, Lihoreau F, Likius A, Mackaye HT, Vignaud P, Brunet $M$ : Cosmogenic nuclide dating of Sahelanthropus tchadensis and Australopithecus bahrelghazali: Mio-Pliocene hominids from Chad. Proc Natl Acad Sci U S A 2008, 105:3226-3323.

106. Kelley J: The hominoid radiation in Asia. In The Primate Fossil Record. Edited by Hartwig WC. Cambridge: Cambridge University Press; 2002:369-384.

107. Leakey MG: Evolution of Theropithecus in the Turkana Basin. In Theropithecus: The Rise and Fall of a Primate Genus. Edited by Jablonski NG. Cambridge: Cambridge University Press; 1993:85-123.

108. Delson E: Cercopithecinae. In Encyclopedia of Human Evolution and Prehistory. Edited by Delson E, Tattersall I, Van Couvering JA, Brooks AS. New York: Garland Publishing Inc; 2000:166-171.

109. Zalmout IS, Sanders WJ, MacLatchy LM, Gunnell GF, Al-Mufarreh YA, Ali MA, Nasser AAH, Al-Masari AM, Al-Sobhi SA, Nadhra AO, Matari AH, Wilson JA, Gingerich PD: New Oligocene primate from Saudi Arabia and the divergence of apes and Old World monkeys. Nature 2010, 466:360-365.

110. Pozzi L, Hodgson JA, Burrell AS, Disotell TR: The stem catarrhine Saadanius does not inform the timing of the origin of crown catarrhines. J Hum Evol 2011, 61:209-210.

111. Stevens NJ, Seiffert ER, O'Connor PM, Roberts EM, Schmitz MD, Krause C, Gorscak E, Ngasala S, Hieronymus TL, Temu J: Palaeontological evidence for an 
Oligocene divergence between Old World monkeys and apes. Nature 2013, 497:611-614.

112. Rambaut A: FigTree: tree figure drawing tool, version 1.4.0. [http://tree.bio.ed.ac.uk/software/figtree/].

113. Lartillot N, Lepage T, Blanquart S: PhyloBayes 3: a Bayesian software package for phylogenetic reconstruction and molecular dating. Bioinformatics 2009, 25:22862288.

114. Thorne JL, Kishino H, Painter IS: Estimating the rate of evolution of the rate of molecular evolution. Mol Biol Evol 1998, 15:1647-1657. 


\title{
Chapter 3
}

\section{Baboon Phylogeny as Inferred From Complete Mitochondrial Genomes}

\author{
Dietmar Zinner, ${ }^{1 \star}$ Jenny Wertheimer, ${ }^{1,2}$ Rasmus Liedigk, ${ }^{2}$ Linn F. Groeneveld, ${ }^{3}$ and Christian Roos ${ }^{2,4}$ \\ ${ }^{1}$ Cognitive Ethology Laboratory, German Primate Center, 37077 Göttingen, Germany \\ ${ }^{2}$ Primate Genetics Laboratory, German Primate Center, 37077 Göttingen, Germany \\ ${ }^{3}$ Courant Research Centre Geobiology, Georg-August-Universität Göttingen, 37077 Göttingen, Germany \\ ${ }^{4}$ Gene Bank of Primates, German Primate Center, 37077 Göttingen, Germany
}

\section{KEY WORDS Papio; evolution; Africa}

\begin{abstract}
Baboons (genus Papio) are an interesting phylogeographical primate model for the evolution of savanna species during the Pleistocene. Earlier studies, based on partial mitochondrial sequence information, revealed seven major haplogroups indicating multiple para- and polyphylies among the six baboon species. The most basal splits among baboon lineages remained unresolved and the credibility intervals for divergence time estimates were rather large. Assuming that genetic variation within the two studied mitochondrial loci so far was insufficient to infer the apparently rapid early radiation of baboons we used complete mitochondria sequence information of ten specimens, representing all major baboon lineages, to reconstruct a baboon phylogeny and to re-estimate divergence times. Our data con-
\end{abstract}

Baboons of the genus Papio have been regarded as a useful analog for hominin behavioral and biological evolution because their evolutionary history took place in parallel to hominins in similar African savanna habitats (Jolly, 1970, 2001; Strum and Mitchell, 1987; Elton, 2006; Codron et al., 2008; Swedell and Plummer, 2012). Extant baboons occur in large parts of sub-Saharan Africa outside the Central- and West African rainforests and are also found in Southwestern Arabia (Jolly, 1993; Kingdon, 1997; Groves, 2001) (Fig. 1a). Representatives of the genus Papio are traditionally divided into five different species or morphotypes, based on morphological, ecological, and behavioral characteristics (Hill, 1970). These are Guinea baboons ( $P$. papio), olive baboons ( $P$. anubis), hamadryas baboons ( $P$. hamadryas), yellow baboons ( $P$. cynocephalus), and chacma baboons (P. ursinus). A similar taxonomic status as for these five forms is warranted for Kinda baboons ( $P$. kindae) (Jolly, 1993, 2001; Frost et al., 2003; Burell, 2008; Zinner et al., 2009). Whether these types should be classified as subspecies of the superspecies $P$. hamadryas (Jolly, 1993) or as distinct species (Groves, 2001; Grubb et al., 2003) is still disputed, but recent studies recognize the six morphotypes as species (Zinner et al., 2011a, 2012).

Various studies tried to clarify the phylogenetic relationships within Papio by analyzing parts of the mitochondrial genome, such as the "Brown region" (Brown et al., 1982) or the cytochrome $b$ gene (Newman et al., 2004; Wildman et al., 2004; Zinner et al., 2009; Keller et al., 2010). Zinner et al. (2009) attempted to resolve the phylogenetic relationships of baboons using both the "Brown region" and the cytochrome $b$ gene, and revealed seven well-supported major haplogroups (Fig. 1b). The

๑ 2012 WILEY PERIODICALS, INC. firmed the earlier tree topology including the para- and polyphyletic relationships of most baboon species; divergence time estimates are slightly younger and credibility intervals narrowed substantially, thus making the estimates more precise. However, the most basal relationships could not be resolved and it remains open whether (1) the most southern population of baboons diverged first or (2) a major split occurred between southern and northern clades. Our study shows that complete mitochondrial genome sequences are more effective to reconstruct robust phylogenies and to narrow down estimated divergence time intervals than only short portions of the mitochondrial genome, although there are also limitations in resolving phylogenetic relationships. Am J Phys Anthropol 000:000-000, 2012. ๑2012 Wiley Periodicals, Inc.

study revealed paraphylies in all species, except for Guinea and Kinda baboons, due to discordances between mitochondrial phylogeny and morphology and/or geographic distribution. However, a strong geographical signal with haplotypes of parapatric populations from different species clustering together was found (Zinner et al., 2009). Although the seven major haplogroups were strongly supported, phylogenetic relationships among them remained largely unresolved. Moreover, divergence time estimates indicated a fast radiation-like (star-like) splitting event into various baboon lineages, starting $\sim 2.09$ million years ago $(\mathrm{Ma})$ (Zinner et al., 2009), which might impede inferring the basal relationships with confidence.

It is plausible that genetic variation within the two studied mitochondrial loci was insufficient to infer the apparently rapid early radiation of baboons. The use of complete mitochondrial sequence information might

Additional Supporting Information may be found in the online version of this article.

Grant sponsor: German Primate Center.

*Correspondence to: Dietmar Zinner, Cognitive Ethology Laboratory, German Primate Center, Kellnerweg 4, 37077 Göttingen, Germany. E-mail: dzinner@gwdg.de

Received 26 June 2012; accepted 8 October 2012

DOI 10.1002/ajpa.22185

Published online in Wiley Online Library

(wileyonlinelibrary.com). 


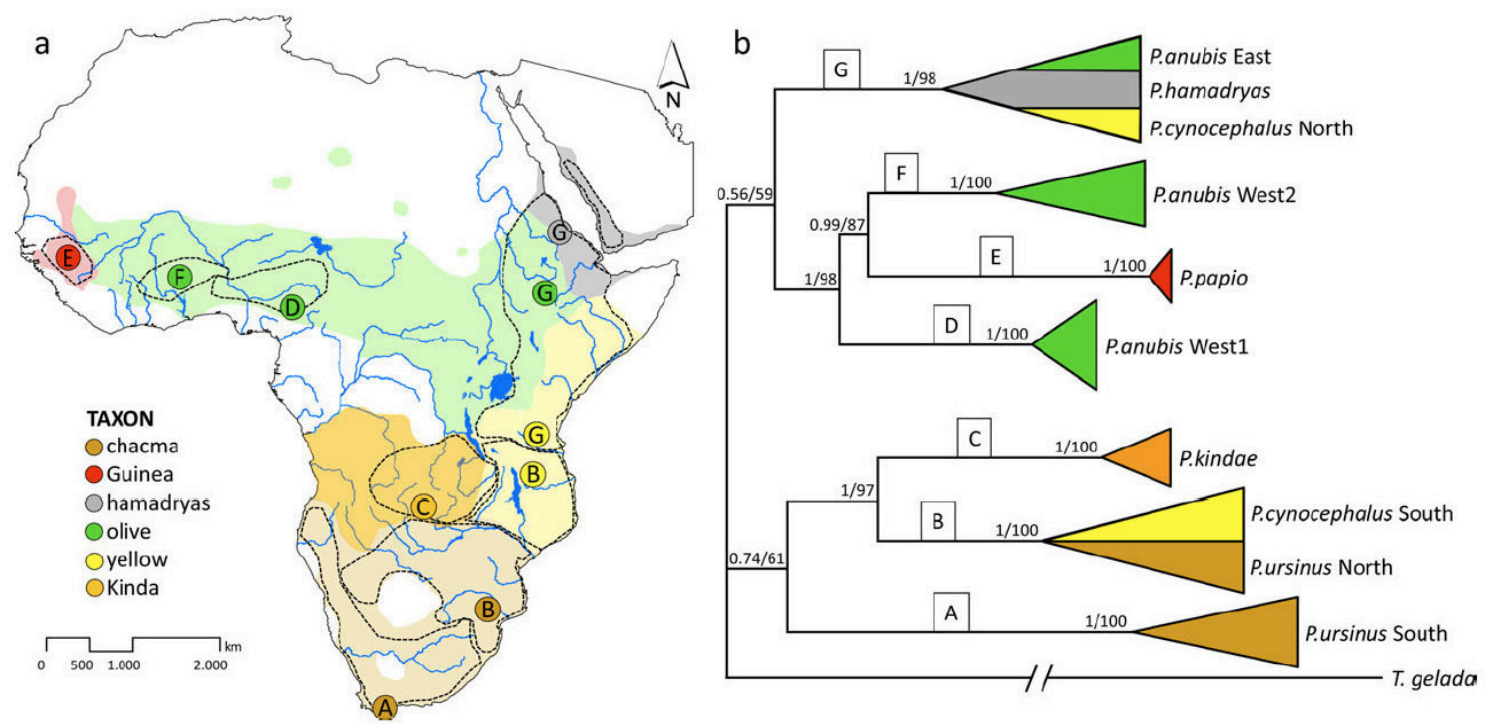

Fig. 1. (a) Distribution of the six baboon species and the seven major mitochondrial haplogroups (A-G) (map based on Kingdon, 1997; Jolly, 2007; Zinner et al., 2009, in press), and (b) simplified phylogenetic relationships among the haplogroups (adapted from Zinner et al., 2009). In (a), dashed lines and colored circles indicate the distribution of the seven major mitochondrial haplogroups and the geographic provenance of samples used in this study (see also Table 1), respectively. In (b), numbers on branches represent support values from Bayesian inference and maximum-likelihood analysis, respectively.

allow a better resolution and stronger statistical support in the phylogenetic tree reconstruction and to narrow down the divergence time credibility intervals (DeFilippis and Moore, 2000; Duchêne et al., 2011; Rokas and Carroll, 2005). A similar approach was successfully applied in recent phylogenetic studies of other taxonomic groups, e.g., gibbons (Chan et al., 2010), colobines (Roos et al., 2011; Liedigk et al., 2012), squirrel monkeys (Chiou et al., 2011); woodpeckers (DeFilippis and Moore, 2000), dolphins (Vilstrup et al., 2011), and bears (Yu et al., 2007). Hence, to test whether sequence information from the complete mitochondrial genome allows a better resolution of phylogenetic relationships and results in smaller divergence time credibility intervals than shorter mitochondrial fragments in baboons, we sequenced and analyzed complete mitochondrial genomes of ten baboons representing all six species and the seven major mitochondrial haplogroups, and compared them with published data.

\section{MATERIALS AND METHODS} Sample collection

We used ten baboon fecal samples, which were collected at various sites in Africa (Fig. 1a and Table 1) for earlier phylogeographic studies (see Zinner et al., 2009 and Keller et al., 2010). We used two types of information to assign samples to respective species: (1) characteristic morphological cues and (2) biogeographic provenance of samples (see Zinner et al., 2009). While observing the animals directly in the field, we used pelage color, body size, general body form, carriage of the tail (curved or "broken") for species identification (after Kingdon, 1997). We further compared the appearance of the baboons in the field with pictures in Kingdon (1997). The ten baboon samples represent all six baboon species and the seven described haplogroups (Fig. 1b, Zinner et al., 2009): P. ursinus South (haplogroup A), P. ursinus North, and $P$. cynocephalus South (haplogroup B), P. kindae (haplogroup C), P. anubis West1 (haplogroup D), P. papio (haplogroup E), P. anubis West2 (haplogroup F), and $P$. anubis East, $P$. hamadryas and $P$. cynocephalus North (haplogroup G). The respective samples were randomly selected from collections of samples of the respective species and haplogroups used in an earlier analysis (Zinner et al., 2009). Fresh fecal material was preserved in $40 \mathrm{ml} 75 \%$ ethanol and dry samples were stored directly on $40 \mathrm{ml}$ silica gel in 50-ml tubes. We stored the samples at ambient temperature for up to 6 months before further processing. The geographic coordinates of the sampling locations were recorded with a GPS.

Our study complied with protocols approved by the respective authorities in countries of origin, and adhered to the legal requirements of the countries in which research was conducted. The study was carried out in compliance with respective animal care regulations and the principles of the American Society of Primatologists and the German Primate Center for the ethical treatment of nonhuman primates.

\section{Laboratory methods}

DNA from fecal material was extracted using the QIAamp DNA Stool Mini Kit (Qiagen, Germany) following manufacturer's protocol with some modifications (Yang et al., 2012). Because of degradation of the DNA extracted from feces, mitochondrial genomes were amplified via 5-25 overlapping fragments, and nested PCRs with an average length of $1,000 \mathrm{bp}$ were sequenced on an $\mathrm{ABI} 3130 x \mathrm{~L}$ sequencer. Respective laboratory methods are outlined in detail in Roos et al. (2011) and Liedigk et al. (2012). To prevent crossindividual contamination, laboratory procedures followed described standards (Roos et al., 2008). Accordingly, DNA extraction, 
BABOON PHYLOGENY

TABLE 1. Baboon samples

\begin{tabular}{lcllrr}
\hline \multicolumn{1}{c}{ Species } & Haplogroup & \multicolumn{1}{c}{ Site } & Country & Long & Lat \\
\hline P. anubis & $\mathrm{G}$ & Managasha NP & Ethiopia & 38.57125 & 8.96838 \\
P. anubis & $\mathrm{D}$ & Komoe NP & Côte d'Ivoire & -3.79000 & 8.80000 \\
P. anubis & $\mathrm{F}$ & Gashaka-Gumti NP & Nigeria & 11.58333 & 7.31667 \\
P. cynocephalus & $\mathrm{G}$ & Mikumi NP & Tanzania & 37.16463 & -7.34651 \\
P. cynocephalus & $\mathrm{B}$ & Amani & Tanzania & 37.51363 & -11.26054 \\
P. hamadryas & $\mathrm{G}$ & Furrus & Eritrea & 38.97115 & 15.01148 \\
P. papio & $\mathrm{E}$ & Niokolo Koba NP & Senegal & -12.76667 & 12.88333 \\
P. kindae & $\mathrm{C}$ & Kasanka NP & Zambia & 30.25202 & -12.59059 \\
P. ursinus & $\mathrm{B}$ & DeHoop NR & South Africa & 20.40658 & -34.45621 \\
P. ursinus & $\mathrm{A}$ & Blyde River & South Africa & 30.79000 & -24.68000 \\
\hline
\end{tabular}

NP: national park; NR: nature reserve.

Species, haplogroup affiliation, geographical location and GenBank accession numbers of studied baboons.

PCR, gel extraction, and sequencing were performed in separate laboratories and randomly repeated after several months, while always only one individual was tested. Sequences from independent analyses were identical. Because only fecal material was used, a contamination of the dataset with nuclear integrations of mitochondrial fragments ("numts") can be regarded as minimal, because nuclear DNA is highly degraded in feces (Thalmann et al., 2004). In fact, test amplifications of the autosomal intron 3 of the serum albumin gene (ALB3) revealed positive PCR amplifications of only $200-400 \mathrm{bp}$ from the 10 baboon DNAs. Further, no multiple amplifications of different copies were detected by direct sequencing of PCR products and overlapping fragments were identical as revealed by visually inspecting electropherograms of all sequences. Complete mitochondrial genome sequences were assembled with GeneiousPro 5.4 (Drummond et al., 2011) and annotated with the online program DOGMA (Wyman et al., 2004). According to DOGMA and manual verification, all protein-coding genes are correctly transcribed, and rRNAs and tRNAs are able to form their typical secondary structure. Sequences were deposited in GenBank (for accession numbers see Table 1).

\section{Statistical analysis}

For phylogenetic analysis, additional orthologous sequences of other primate taxa deposited in GenBank were added. The final dataset comprised 18 sequences including ten baboons, four other cercopithecines (Theropithecus gelada [FJ785426], Macaca sylvanus [A.J309865], M. mulatta [JQ821843], Chlorocebus aethiops [AY863426]), one colobine (Colobus guereza [AY863427]), and three hominoid species (Homo sapiens [X93334], Pan troglodytes [D38113], Pongo abelii [X97707]), that were used as outgroup taxa. Sequences were aligned with Muscle 3.7 (Edgar, 2004) and manually corrected. For phylogenetic analyses, two different datasets were generated. The first dataset (mtDNA1) consists of the complete mitochondrial genome in which only poorly aligned positions and indels were removed with Gblocks 0.91b (Castresana, 2000) using default settings. The second dataset (mtDNA2), generated in Mesquite 2.75 (Maddison and Maddison, 2011), included only the 12 protein-coding genes on the heavy strand.

Phylogenetic tree reconstructions were conducted with maximum-likelihood (ML) and Bayesian algorithms, using respectively the programs GARLI 2.0 (Zwickl, 2006) and MrBayes 3.1.2 (Huelsenbeck et al., 2001; Ronquist and Huelsenbeck, 2003). For all reconstructions, the best-fit model of nucleotide substitution was chosen with the Bayesian information criterion (BIC) in jModeltest 2.1 (Posada, 2009) (Supporting Information Table S1). For the mtDNA2 dataset, each locus was treated separately and each with its own substitution model. For ML reconstructions in GARLI, only the models were specified, while all other settings were left at their default value. Respective internal node support was assessed by bootstrap analyses with 500 replicates and majority-rule consensus trees were calculated in PAUP* $4.0 \mathrm{~b} 10$ (Swofford, 2003). For Bayesian analyses, we applied four independent Markov Chain Monte Carlo (MCMC) runs with the default temperature of 0.2 . We ran four repetitions for 10 million generations with tree and parameter sampling every 100 generations. Acceptance rates were in the optimal range of $10-70 \%$. The adequacy of a $25 \%$ burn-in and convergence of all parameters was checked via the uncorrected potential scale reduction factor (PSRF) (Gelman and Rubin, 1992) as estimated by MrBayes and by inspecting the trace of the parameters across generations using the software TRACER 1.5 (Rambaut and Drummond, 2007). Whether posterior split probabilities were also converging was examined with AWTY (Nylander et al., 2008). Posterior probabilities and a phylogram with mean branch lengths were calculated from the posterior density of trees. Alternative phylogenetic positions of the $P$. ursinus South haplogroup among baboons, and various alternative relationships among the $P$. anubis West1, $P$. anubis West2 and $P$. papio haplogroups were evaluated with the Kishino-Hasegawa (Kishino and Hasegawa, 1989) and Shimodaira-Hasegawa (Shimodaira and Hasegawa, 1999) tests with full optimization and 1,000 bootstrap replicates in PAUP*.

Divergence ages from both mitochondrial datasets were estimated in BEAST 1.6.1 (Drummond and Rambaut, 2007) with a relaxed molecular clock approach (Drummond et al., 2006). Therefore, we assumed a relaxed lognormal model of lineage variation and a birth-death process prior for branching rates. The mtDNA2 dataset was partitioned treating each locus separately and each with its own substitution model, while dataset mtDNA1 was regarded as one partition. Five fossil-based calibration points were applied with a normal distribution prior for respective nodes: the split between Homo and Pan 6.5 Ma with a 95\% credibility interval (CI) of $0.5 \mathrm{Ma}$ (Vignaud et al., 2002; Brunet et al., 2005; Lebatard et al., 2008), the separation of Pongo from the Homo + Pan lineage $14 \mathrm{Ma}$ and a 95\% CI of 1.0 Ma (Kelley, 2002), the split between Theropithecus and Papio 4 Ma (95\% CI: $0.5 \mathrm{Ma}$ ) (Leakey, 1993; 


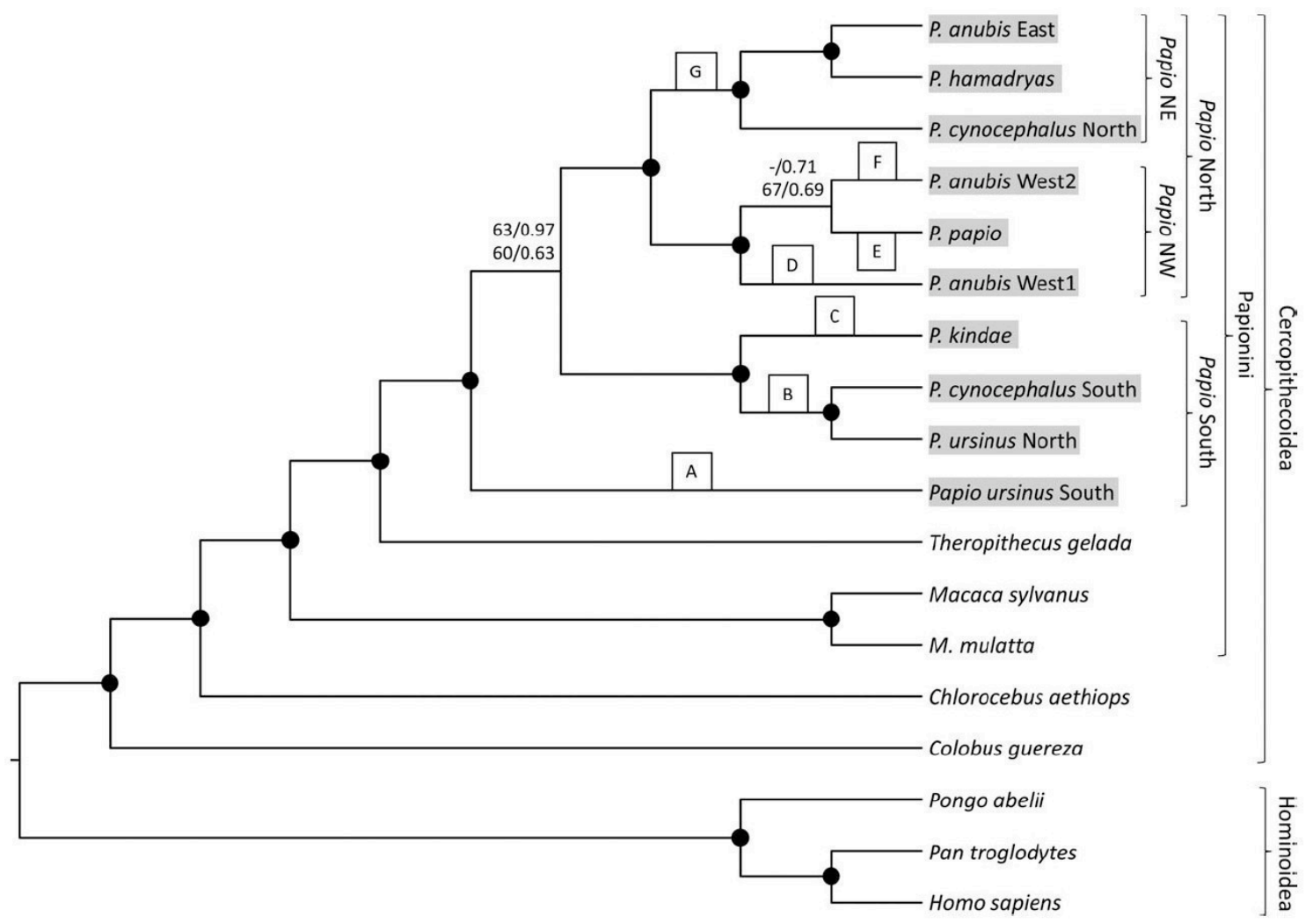

Fig. 2. Phylogenetic relationships among baboons and outgroup taxa based on complete mitochondrial genome sequences. Black dots on nodes indicate ML bootstrap values of $99-100 \%$ and Bayesian posterior probabilities of 1.0; values below are given at respective branches (upper line: mtDNA1, lower line: mtDNA2). A-G indicates the seven major haplogroups in Papio according to Zinner et al. (2009).

Delson, 2000), the split between M. sylvanus and $M$. mulatta 5.5 Ma (95\% CI: 0.5 Ma) (Delson, 1980), and the divergence of hominoids and cercopithecoids $26.5 \mathrm{Ma}$ (95\% CI: $2.5 \mathrm{Ma}$ ) (Zalmout et al., 2010; Pozzi et al., 2011). We ran four replicates for 25 million generations with tree and parameter sampling occurring every 1,000 generations. TRACER was used to assess the adequacy of a $10 \%$ burn-in and convergence of all parameters across generations. Subsequently, sampling distributions were combined ( $25 \%$ burn-in) with the software LogCombiner 1.6.1 and a consensus chronogram with node height distribution was generated and visualized with TreeAnnotator 1.6.1 and FigTree 1.3.1 (Rambaut, 2008).

\section{RESULTS}

Mitochondrial genome sequences were successfully amplified and sequenced from ten Papio individuals. All sequenced genomes consisted of 13 protein-coding genes, 2 rRNA genes, 22 tRNA genes, and the control region. The initial alignment comprising a total of 18 sequences had a length of $16,858 \mathrm{bp}$. After removing poorly aligned positions and indels, the mtDNA1 dataset had a length of $16,055 \mathrm{bp}$, while the mtDNA2 dataset, including only the 12 protein-coding genes on the heavy strand, was $10,854 \mathrm{bp}$ in length.

Phylogenetic trees obtained from both datasets (mtDNA1 and mtDNA2) and derived from Bayesian in- ference and the ML algorithm yielded identical tree topologies and mainly well supported branching patterns (ML bootstrap values: 99-100\%, Bayesian posterior probabilities: 1.0) (Fig. 2). The only exceptions are the phylogenetic position of $P$. ursinus South, and the relationships among the two western $P$. anubis and $P$. papio. According to our tree reconstructions, $P$. ursinus South diverged first and appears as sister lineage to all other baboons. However, statistical support is weak (ML bootstrap values: $60-63 \%$, Bayesian posterior probabilities: 0.63-0.97) and alternative positions of $P$. ursinus South within the baboon clade (sister to either the southern or northern clades, or an unresolved trichotomy among these two clades and $P$. ursinus South) are statistically not rejected $(P>0.05)$. After this initial split, a major division occurred between the remaining southern and the northern lineages. Among the southern lineages, $P$. kindae split off before $P$. ursinus North and $P$. cynocephalus South separated. The northern lineages first divided into a northwestern and a northeastern clade. Within the latter, $P$. cynocephalus North diverged first from a clade consisting of $P$. anubis East and $P$. hamadryas. In the northwestern clade, $P$. anubis West 1 is suggested to be the sister lineage to the $P$. anubis West2-P. papio clade. However, statistical support for this relationship is weak (ML bootstrap values: $<50-67 \%$, Bayesian posterior probabilities: $0.69-0.71$ ) and alternative relationships ( $P$. anubis West1 and West2 are sister taxa, 
BABOON PHYLOGENY

TABLE 2. Estimation of divergence ages in Ma (95\% credibility intervals)

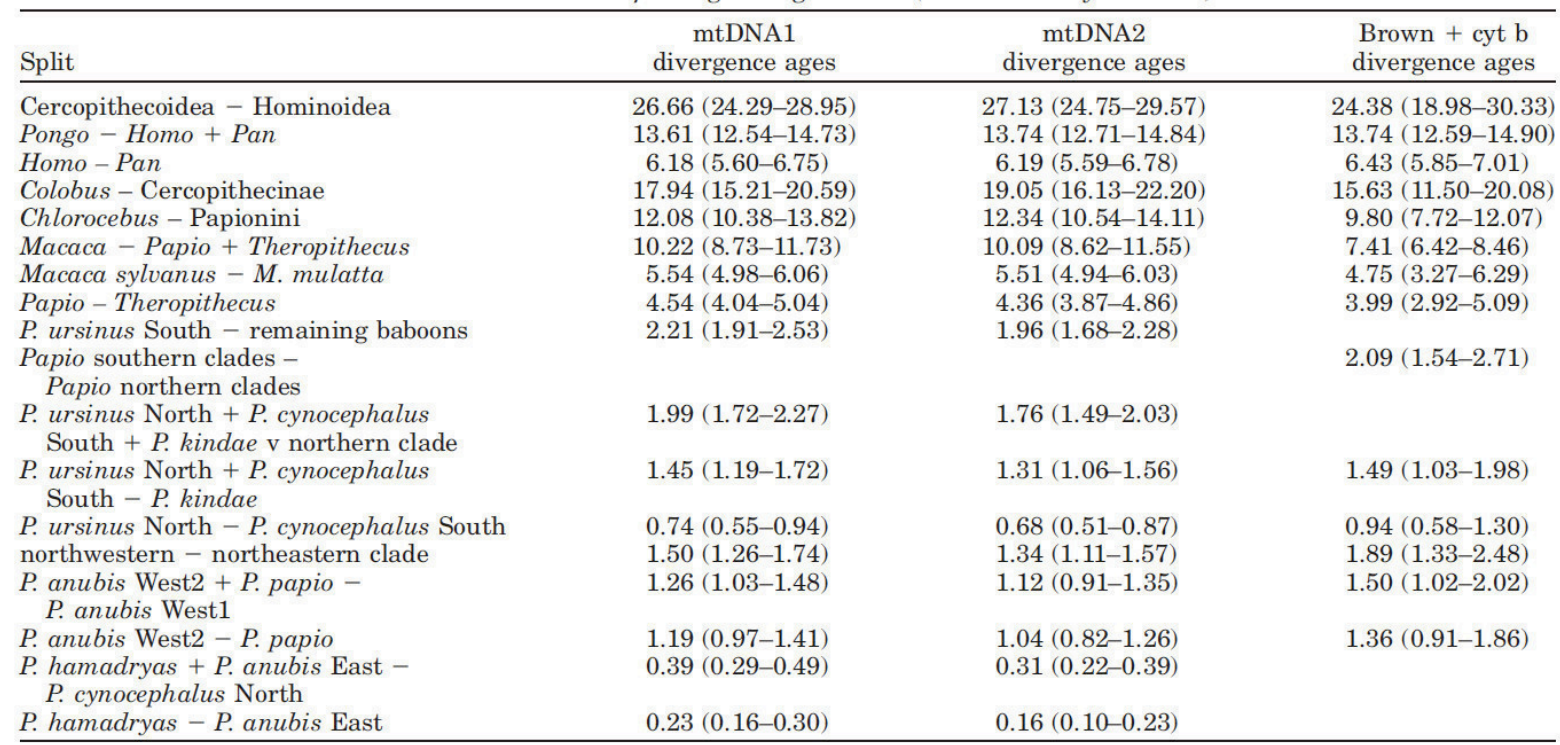

Estimates based on "Brown region" and cytochrome $b$ sequence information are taken from Zinner et al. (2009).

$P$. anubis West1 is sister taxon to $P$. papio, or an unresolved trichotomy among the three lineages) are statistically not rejected $(P>0.05)$.

Divergence age estimates obtained from both datasets are highly similar albeit estimates from the mtDNA2 dataset are generally slightly younger than from the mtDNA1 dataset (Table 2). Accordingly, the divergence of Papio into the seven major haplogroups started 1.96-2.21 Ma and ended $0.68-0.74 \mathrm{Ma}$ (for $95 \%$ credibility intervals see Table 2). The three lineages in haplogroup $\mathrm{G}$ diverged 0.31-0.39 and 0.16-0.23 Ma, respectively, and the two lineages in haplogroup B separated 0.68-0.74 Ma.

\section{DISCUSSION}

We aimed to find stronger statistical support in the phylogenetic tree reconstruction of Papio mitochondrial DNA, in particular for the most basal splits, and to narrow down the divergence time credibility intervals by basing our study on whole mitochondrial genomes. The second aim was accomplished by using complete mitochondrial genome information, whereas the resolution of the basal splits remained ambiguous.

The Papio phylogeny based on whole mitochondrial genome sequences shows a highly similar tree topology as the phylogeny based on sequences of the cytochrome $b$ gene and the "Brown region" (Zinner et al., 2009, 2011a; Keller et al., 2010), but with stronger support for most nodes. Para- and polyphyletic relationships for almost all baboon species were confirmed. One possible cause for the presence of para- and polyphylies are "numts." They can be excluded here, because overlapping parts of the various amplification fragments were identical, and because of the correct translation of protein-coding genes and the forming of typical secondary structures of tRNAs and rRNAs (Thalmann et al., 2004). Incomplete lineage sorting is also highly unlikely, because this process should be random in respect to geography (Avise, 2004). However, in our mitochondrial phylogeny, we found that geographic close lineages cluster together, and, hence, introgressive hybridization remains as the most probable process leading to the observed phylogenetic discordances (Funk and Omland, 2003; Burell 2008; Zinner et al., 2009, 2011b; Jolly et al., 2011).

Estimated divergence times for all nodes are in a similar range as dates from other molecular studies (Chan et al., 2010; Perelman et al., 2011; Roos et al., 2011; Liedigk et al., 2012; Steiper and Seiffert, 2012). Also among baboon lineages, estimated divergence times are generally consistent with previous work by Zinner et al. (2009), but appear slightly younger (Table 2). The respective credibility intervals have narrowed to $<55 \%$. For example, the breadth of the credibility interval around the northeastern and northwestern divergence, estimated at $1.50 \mathrm{Ma}$ from dataset mtDNA1 and 1.34 Ma from dataset mtDNA2, is reduced to $95 \%$ CIs of only 0.48 and $0.46 \mathrm{Ma}$, respectively, and thus less wide than the $95 \%$ CI of $1.15 \mathrm{Ma}$ around the previously estimated divergence time of 1.89 Ma (Zinner et al., 2009).

Although the tree topology in general is strongly supported by both algorithms, some relationships identified as weakly supported in earlier studies remained ambiguous (relationship among the three western clades, $P$. anubis West1, $P$. anubis West2, and $P$. papio, and the relationship between $P$. ursinus South and all other clades). The origin of the genus lies most likely in southern Africa. This is in agreement with earlier suggestions from mitochondrial phylogenies (Newman et al., 2004; Sithaldeen et al., 2009; Zinner et al., 2009; Keller et al., 2010) and fossil data (Jablonski and Frost, 2010; Williams et al., 2012). However, the chronology of the initial divergences in southern Africa is not clear, because alternative scenarios are statistically not rejected. In our reconstruction, $P$. ursinus South diverged first followed by the main southnorth split, but similarly possible are an initial southnorth split or a trifurcation within a relative short time period (mtDNA1: 2.21-1.99 Ma; mtDNA2: $1.96-1.76 \mathrm{Ma}$ ) among the lineages leading to $P$. ursinus South, the clade consisting of $P$. ursinus North, $P$. cynocephalus South and $P$. kindae, and the northern clade. 


\section{ZINNER ET AL.}

The refined divergence dates among baboon mitochondrial lineages do not contradict the earlier hypothesis that the timing of divergence events among Papio lineages can be placed in a wider context related to changes in the African paleoclimate during the Pleistocene with recurrent expansions and retreats of the savanna biome as suitable habitat for baboons (Hamilton and Taylor, 1991; deMenocal, 1995, 2004; Maley, 1996; Zinner et al., 2009, 2011a, Bettridge and Dunbar, 2012). Because of periodical climate changes and the isolation and reconnection of savanna habitats, populations of baboons changed in size and spatial distribution, perhaps cyclically. Gene flow between populations occurred at differing degrees and at different times, leading to the phylogeographic pattern of baboons observed today (Zinner et al. 2009, 2011a). Although key aspects of African faunal evolution in relation to climate change remain poorly understood (Bobé et al., 2002), previous studies have already pointed out the role of glacial and interglacial changes for refugial differentiation and migration routes for the African continent (Arctander et al., 1999; Nersting and Arctander, 2001; Nyakaana et al., 2002; Lorenzen et al., 2010, 2012).

We are aware of the fact that with only mitochondrial DNA information at hand and the indications of excessive introgression, we cannot say much about the taxonomic level of the various baboon forms. However, a classification of baboon taxa as subspecies instead of species or as members of a superspecies would not change the general problem of finding closely related mitochondrial DNA in different taxa or largely different mitochondrial DNA in the same taxon. We would just shift the problem to another taxonomic level.

In conclusion, the general tree topology including paraphyletic relationships of most baboon species (Newman et al., 2004; Zinner et al., 2009; Keller et al., 2010) was confirmed by the use of whole mitochondrial sequence information and divergence times among baboon mitochondrial lineages became more reliable. This might have consequences for the use of Papio baboons as an analogous phylogeographic model for intra-African dispersal of hominins during the Pleistocene (Lahr and Foley, 1998). However, to fully elucidate the putative complex evolutionary history of baboon taxa and to confirm hybridization events among them, large-scale nuclear sequence data are needed.

\section{ACKNOWLEDGMENTS}

The authors thank Christina Keller, Dawit Berhane, Britta Kunz, Umaru Buba, and Julius W. Nyahongo for helping collecting samples; Christiane Schwarz for her help in the laboratory, and Tanja Haus and Gisela Fickenscher for their critical comments on an earlier version of the paper. They also thank the University of Dodoma, TAWIRI and COSTECH (Tanzania), Cape Nature House (South Africa), ZAWA (Zambia), the Direction Parcs Nationaux (Senegal), the Ministère de l'Agriculture et des Ressources Animales (Côte d'Ivoire), Gashaka Primate Project (Nigeria) and the Ethiopian Wildlife Conservation Organization for their help during sample collection.

\section{LITERATURE CITED}

Arctander P, Johansen C, Coutellec-Vreto MA. 1999. Phylogeography of three closely related African bovids (tribe Alcelaphini). Mol Biol Evol 16:1724-1739.
Avise J. 2004. Molecular markers, natural history, and evolution. Sunderland, MA: Sinauer Associates.

Bettridge C, Dunbar RIM. 2012. Modeling the biogeography of fossil baboons. Int J Primatol Online.

Bobé R, Bersemeyer AK, Chapman RE. 2002. Faunal change, environmental variability and late Pliocene hominin evolution. J Hum Evol 42:475-497.

Brown WM, Prager EM, Wang A, Wilson AC. 1982. Mitochondrial DNA sequences of primates: tempo and mode of evolution. J Mol Evol 18:225-239.

Brunet M, Guy F, Pilbeam D, Lieberman DE, Likius A, Mackaye HT, Ponce de León MS, Zollikofer CP, Vignaud P. 2005. New material of the earliest hominid from the Upper Miocene of Chad. Nature 434:752-755.

Burrell AS. 2008. Phylogenetics and population genetics of Central African baboons. PhD Thesis. New York: New York University.

Castresana J. 2000. Selection of conserved blocks from multiple alignments for their use in phylogenetic analysis. Mol Biol Evol 17:540-552.

Chan YC, Roos C, Inoue-Murayama M, Inoue E, Shih CC, Pei KJC, Vigilant L. 2010. Mitochondrial genome sequences effectively reveal the phylogeny of Hylobates gibbons. PLoS ONE 5:e14419.

Chiou KL, Pozzi L, Lynch Alfaro JW, Di Fiore A. 2011. Pleistocene diversification of living squirrel monkeys (Saimiri spp.) inferred from complete mitochondrial genome sequences. Mol Phylogenet Evol 59:736-745.

Codron D, Lee-Thorp JA, Sponheimer M, de Ruiter D, Codron J. 2008. What insights can baboon feeding ecology provide for early Hominin niche differentiation? Int J Primatol 29:757-772.

DeFilippis VR, Moore WS. 2000. Resolution of phylogenetic relationships among recently evolved species as a function of amount of DNA sequence: an empirical study based on woodpeckers (Aves: Picidae). Mol Phylogenet Evol 16:143-160.

Delson E. 1980. Fossil macaques, phyletic relationships and a scenario of deployment. In: Lindburg DG, editor. The macaques: studies in ecology, behavior and evolution. New York: Van Nostrand Reinhold. p 10-30.

Delson E. 2000. Cercopithecinae. In: Delson E, Tattersall I, Van Couvering JA, Brooks AS, editors. Encyclopedia of human evolution and prehistory. New York: Garland. p 166-171.

deMenocal PB. 1995. Plio-Pleistocene African climate. Science 270:53-59.

deMenocal PB. 2004. African climate change and faunal evolution during the Pliocene Pleistocene. Earth Planet Sci Lett 220:3-24.

Drummond AJ, Ashton B, Buxton S, Cheung M, Cooper A, Duran C, Field M, Heled J, Kearse M, Markowitz S, Moir R, Stones-Havas S, Sturrock S, Thierer T, Wilson A. 2011. Geneious, version 5.4. Geneious website. Available at: http:// www.geneious.com. Accessed 2011 Jan 15.

Drummond AJ, Ho SYW, Phillips MJ, Rambaut A. 2006 Relaxed phylogenetics and dating with confidence. PLoS Biol 4:e88.

Drummond AJ, Rambaut A. 2007. BEAST: Bayesian evolutionary analysis by sampling trees. BMC Evol Biol 7:214.

Duchêne S, Archer F, Vilstrup J, Caballero S, Morin P. 2011. Mitogenome phylogenetics: the impact of using single regions and partitioning schemes on topology, substitution rate and divergence time estimation. PLoS ONE 6:e27138.

Edgar RC. 2004. MUSCLE: multiple sequence alignment with high accuracy and high throughput. Nucleic Acids Res 32: 1792-1797.

Elton S. 2006. Forty years on and still going strong: the use of hominin-cercopithecid comparisons in palaeoanthropology. J R Anthropol Inst (NS) 12:19-38.

Frost SR, Marcus LF, Bookstein FL, Reddy DP, Delson E. 2003. Cranial allometry, phylogeography, and systematics of largebodied papionins (primates: Cercopithecinae) inferred from geometric morphometric analysis of landmark data. Anat Rec 275A:1048-1072.

Funk DJ, Omland KE. 2003. Species-level paraphyly and polyphyly: frequency, causes, and consequences, with insights 
from animal mitochondrial DNA. Annu Rev Ecol Evol Syst 34:397-423.

Gelman A, Rubin D. 1992. Inference from iterative simulation using multiple sequences. Stat Sci 7:457-511.

Groves C. 2001. Primate taxonomy. Washington, DC: Smithsonian Institution Press.

Grubb P, Butynski TM, Oates JF, Bearder SK, Disotell TR, Groves C, Struhsaker TT. 2003. Assessment of the diversity of African primates. Int J Primatol 24:1301-1357.

Hamilton AC, Taylor D. 1991. History of climate and forests in tropical Africa during the last 8 million years. Climatic Change 19:65-78.

Hill WCO. 1970. Primates: comparative anatomy and taxonomy. Cynopithecinae: Papio, Mandrillus, Theropithecus. Edinburgh: Edinburgh University Press.

Huelsenbeck JP, Ronquist F, Nielsen R, Bollback JP. 2001. Bayesian inference of phylogeny and its impact on evolutionary biology. Science 294:2310-2314.

Jablonski NG, Frost SR. 2010. Cercopithecoidea. In: Werdelin L, Sanders W, editors. Cenozoic mammals of Africa. Berkeley: University of California Press. p 393-428.

Jolly CJ. 1970. The seed-eaters: a new model of hominid differentiation based on a baboon analogy. Man 5:1-26.

Jolly CJ. 1993. Species, subspecies, and baboon systematics. In: Kimbel WH, Martin LB, editors. Species, species concepts, and primate evolution. New York: Plenum Press. p 67-101.

Jolly CJ. 2001. A proper study for mankind: analogies from the Papionin monkeys and their implications for human evolution. Yearb Phys Anthropol 44:177-204.

Jolly CJ. 2007. Baboons, mandrills, and mangabeys: Afro-Papionin socioecology in a phylogenetic perspective. In: Campbell CJ, Fuentes A, MacKinnon KC, Panger M, Bearder SK, editors. Primates in perspective. New York: Oxford University Press. p 240-251.

Jolly CJ, Burrell AS, Phillips-Conroy JE, Bergey CM, Rogers J. 2011. Kinda baboons (Papio kindae) and grayfoot chacma baboons ( $P$. ursinus griseipes) hybridize in the Kafue River Valley, Zambia. Am J Primatol 73:291-303.

Keller C, Roos C, Groeneveld LF, Fischer J, Zinner D. 2010. Introgressive hybridization in southern African baboons shapes patterns of mtDNA variation. Am J Phys Anthropol 142:125-136.

Kelley J. 2002. The hominoid radiation in Asia. In: Hartwig WC, editor. The primate fossil record. Cambridge: Cambridge University Press. p 369-384.

Kingdon J. 1997. The Kingdon field guide to African mammals. London: Academic Press.

Kishino H, Hasegawa M. 1989. Evaluation of the maximum likelihood estimate of the evolutionary tree topologies from DNA sequence data, and the branching order in Hominoidea. J Mol Evol 29:170-179.

Lahr MM, Foley RA. 1998. Toward a theory of modern human origins: geography, demography and diversity in recent human evolution. Yearb Phys Anthropol 41:137-176.

Leakey MG. 1993. Evolution of Theropithecus in the Turkana Basin. In: Jablonski NG, editor. Theropithecus, the rise and fall of a primate genus. Cambridge: Cambridge University Press. p 85-124.

Lebatard AE, Bourlès DL, Duringer P, Jolivet M, Braucher R, Carcaillet J, Schuster M, Arnaud N, Monié P, Lihoreau F, Likius A, Mackaye HT, Vignaud P, Brunet M. 2008. Cosmogenic nuclide dating of Sahelanthropus tchadensis and Australopithecus bahrelghazali: Mio-Pliocene hominids from Chad. Proc Natl Acad Sci USA 105:3226-3231.

Liedigk R, Yang M, Jablonski NG, Momberg F, Geissmann T, Lwin N, Htin Hla T, Liu Z, Wong B, Li M, Yongcheng L, Zhang Y-P, Nadler T, Zinner D, Roos C. 2012. Evolutionary history of the odd-nosed monkeys and the phylogenetic position of the newly described Myanmar snub-nosed monkey Rhinopithecus strykeri. PLoS ONE 7:e37418.

Lorenzen E, Heller R, Siegismund H.R. 2012. Comparative phylogeography of African savannah ungulates. Mol Ecol 21: 3656-3670.

Lorenzen E, Masembe C, Arctander P, Siegismund HR. 2010. A long-standing Pleistocene refugium in southern Africa and a mosaic of refugia in East Africa: insights from mtDNA and the common eland antelope. J Biogeogr 37:571-581.

Maddison WP, Maddison DR. 2011. Mesquite: a modular system for evolutionary analysis, version 2.75. Available at: http:// mesquiteproject.org. Accessed 2011 March 11.

Maley J. 1996. The African rain forest: main characteristics of changes in vegetation and climate from the Upper Cretaceous to the Quaternary. In: Alexander IJ, Swaine MD, Watling R, editors. Essays on the ecology of the Guinea-Congo rain forest. Proc R Soc Edinburgh 104B:31-73.

Nersting LG, Arctander P. 2001. Phylogeography and conservation of impala and greater kudu. Mol Ecol 10:711-719.

Newman TK, Jolly CJ, Rogers J. 2004. Mitochondrial phylogeny and systematics of baboons (Papio). Am J Phys Anthropol 124:17-27.

Nyakaana S, Arctander P, Siegismund HR. 2002. Population structure of the African savannah elephant inferred from mitochondrial control region sequences and nuclear microsatellite loci. Heredity 89:90-98.

Nylander JA, Wilgenbusch JC, Warren DL, Swofford DL. 2008 AWTY (are we there yet?): a system for graphical exploration of MCMC convergence in Bayesian phylogenetics. Bioinformatics 24:581-583.

Perelman P, Johnson WE, Roos C, Seuánez HN, Horvath JE, Moreira MA, Kessing B, Pontius J, Roelke M, Rumpler Y, Schneider MP, Silva A, O'Brien SJ, Pecon-Slattery J. 2011. A molecular phylogeny of living primates. PLoS Genet 7:e1001342.

Posada D. 2009. Selection of models of DNA evolution with jModelTest. Methods Mol Biol 537:93-112.

Pozzi L, Hodgson JA, Burrell AS, Disotell TR. 2011. The stem catarrhine Saadanius does not inform the timing of the origin of crown catarrhines. J Hum Evol 61:209-210.

Rambaut A. 2008. FigTree: tree figure drawing tool, version 1.2.2. FigTree website. Available at: http://tree.bio.ed.ac.uk/ software/figtree/. Accessed 2008 Dec 18.

Rambaut A, Drummond AJ. 2007. Tracer: MCMC trace analysis tool, version 1.5. Tracer website. Available at: http://tree.bio.ed.ac.uk/sofware/tracer/. Accessed 2008 Feb 11.

Rokas A, Carroll SB. 2005. More genes or more taxa? The relative contribution of gene number and taxon number to phylogenetic accuracy. Mol Biol Evol 22:1337-1344.

Ronquist F, Huelsenbeck JP. 2003. MrBayes 3: Bayesian phylogenetic inference under mixed models. Bioinformatics 19:1572-1574.

Roos C, Nadler T, Walter L. 2008. Mitochondrial phylogeny, taxonomy and biogeography of the silvered langur species group (Trachypithecus cristatus). Mol Phylogenet Evol 47:629-636.

Roos C, Zinner D, Kubatko LS, Schwarz C, Yang M, Meyer D, Nash SD, Xing J, Batzer MA, Brameier M, Leendertz FH, Ziegler T, Perwitasari-Farjallah D, Nadler T, Walter L, Osterholz M. 2011. Nuclear versus mitochondrial DNA: evidence for hybridization in colobine monkeys. BMC Evol Biol 11:77.

Shimodaira H, Hasegawa M. 1999. Multiple comparisons of loglikelihoods with applications to phylogenetic inference. Mol Biol Evol 16:1114-1116.

Sithaldeen R, Bishop JM, Ackermann RR. 2009. Mitochondrial DNA analysis reveals Plio-Pleistocene diversification within the chacma baboon. Mol Phylogenet Evol 53:1042-1048.

Steiper ME, Seiffert ER. 2012. Evidence for a convergent slowdown in primate molecular rates and its implications for the timing of early primate evolution. Proc Natl Acad Sci USA 109:6006-6011.

Strum SC, Mitchell W. 1987. Baboon models and muddles. In: Kinzey WG, editor. The evolution of human behavior: primate models. New York: New York State University Press. p 87-104.

Swedell L, Plummer T. 2012. A papionin multilevel society as a model for hominin social evolution. Int J Primatol 33:1165-1193. Swofford DL. 2003. PAUP*: phylogenetic analysis using parsimony (*and other methods), version 4. Sunderland, MA: Sinauer Associates.

Thalmann O, Hebler J, Poinar HN, Pääbo S, Vigilant L. 2004. Unreliable mtDNA data due to nuclear insertions: a cautionary tale from analysis of humans and other great apes. Mol Ecol 13:321-325. 
Vignaud P, Duringer P, Mackaye HT, Likius A, Blondel C, Boisserie JR, De Bonis L, Eisenmann V, Etienne ME, Geraads D, Guy F, Lehmann T, Lihoreau F, Lopez-Martinez N, Mourer-Chauviré C, Otero O, Rage JC, Schuster M, Viriot L, Zazzo A, Brunet M. 2002. Geology and palaeontology of the Upper Miocene Toros-Menalla hominid locality, Chad. Nature 418: $152-155$.

Vilstrup JT, Ho SYW, Foote AD, Morin PA, Kreb D, Krützen M, Parra GJ, Robertson KM, de Stephanis R, Verborgh P Willerslev E, Orlando L, Gilbert MTP. 2011. Mitogenomic phylogenetic analyses of the Delphinidae with an emphasis on the Globicephalinae. BMC Evol Biol 11:65.

Wildman DE, Bergman TJ, al-Aghbari A, Sterner KN, Newman TK, Phillips-Conroy JE, Jolly CJ, Disotell TR. 2004. Mitochondrial evidence for the origin of hamadryas baboons. Mol Phylogenet Evol 32:287-296.

Williams BA, Ross CF, Frost SR, Waddle DM, Gabadirwe M, Brook GA. 2012. Fossil Papio cranium from !Ncumtsa (Koanaka) Hills, western Ngamiland, Botswana. Am J Phys Anthropol 149:1-17.

Wyman SK, Jansen RK, Boore JL. 2004. Automatic annotation of organellar genomes with DOGMA. Bioinformatics 20: 3252-3255.

Yang M, Yang Y, Cui D, Fickenscher G, Zinner D, Roos C, Brameier M. 2012. Population genetic structure of Guizhou snub-nosed monkeys (Rhinopithecus brelichi) as inferred from mitochondrial control region sequences, and comparison with $R$. roxellana and R. bieti. Am J Phys Anthropol 147:1-10.
Yu L, Li YW, Ryder O, Zhang YP. 2007. Analysis of complete mitochondrial genome sequences increases phylogenetic resolution of bears (Ursidae), a mammalian family that experienced rapid speciation. BMC Evol Biol 7:198.

Zalmout IS, Sanders WJ, MacLatchy LM, Gunnell GF, AlMufarreh YA, Ali MA, Nasser AA, Al-Masari AM, Al-Sobhji SA, Nadhra AO, Matari AH, Wilson JA, Gingerich PD. 2010. New Oligocene primate from Saudi Arabia and the divergence of apes and Old World monkeys. Nature 466:360-365.

Zinner D, Arnold ML, Roos C. 2011b. The strange blood: natural hybridization in primates. Evol Anthropol 20:96-103.

Zinner D, Buba U, Nash S, Roos C. 2011a. Pan-African voyagers.

The phylo-geography of baboons. In: Sommer V, Ross C, editors. Primates of Gashaka. Socioecology and conservation in Nigeria's biodiversity hotspot. New York: Springer. p 267-306.

Zinner D, Fickenscher G, Anandam M, Bennett EL, Davenport TRB, Davies N, Engelhardt A, Eudey AA, Gadsby EL, Groves CP, Healy A, Karanth KP, Molur S, Nadler T, Richardson M, Riley EP, Sheeran LK, Ting N, Wallis J, Waters S, Whittaker D, Roos C. Old World monkeys (Cercopithecidae). In: Mittermeier RA, Wilson DE, editors. Handbook of the mammals of the world . Vol.3. Primates. Barcelona: Lynx Edicions (in press).

Zinner D, Groeneveld LF, Keller C, Roos C. 2009. Mitochondrial phylogeography of baboons (Papio spp.): indication for introgressive hybridization? BMC Evol Biol 9:83.

Zwickl DJ. 2006. Genetic algorithm approaches for the phylogenetic analysis of large biological sequence data sets under the maximum likelihood criterion. PhD Thesis. Austin: University of Texas. 


\section{Chapter 4}

\section{Mitogenomic phylogeny and phylogeography of the common long-tailed macaque (Macaca fascicularis fascicularis)}

Rasmus Liedigk $^{1 *}$, Jakob Kolleck${ }^{1}$, Kai O. Böker ${ }^{1,2}$, Erik Meijaard ${ }^{3,4,5}$, Badrul Munir Md-Zain ${ }^{6}$, Muhammad Abu Bakar Abdul-Latiff ${ }^{6}$, Ahmad Ampeng ${ }^{7}$, Maklarin Lakim ${ }^{8}$, Pazil Abdul-Patah ${ }^{9}$, Markus Brameier ${ }^{1}$, Dietmar Zinner ${ }^{10}$, Christian Roos ${ }^{1,11}$

1 Primate Genetics Laboratory, German Primate Center, Leibniz Institute for Primate Research, Goettingen, Germany; ${ }^{2}$ Junior Research Group Medical RNA Biology, Primate Genetics Laboratory, German Primate Center, Leibniz Institute for Primate Research, Goettingen, Germany; ${ }^{3}$ Borneo Futures Project, People and Nature Consulting International, Jakarta, Indonesia; ${ }^{4}$ School of Archaeology \& Anthropology, Australian National University, Canberra, Australia; ${ }^{5}$ School of Biological Sciences, University of Queensland, St Lucia, Australia; ${ }^{6}$ School of Environmental and Natural Resource Sciences, Faculty of Science and Technology, Universiti Kebangsaan Malaysia, Bangi, Selangor, Malaysia; ${ }^{7}$ Sarawak Forest Department, Kuching, Sarawak, Malaysia; ${ }^{8}$ Sabah Parks, Research and Education Division, Kota Kinabalu, Sabah, Malaysia;

${ }^{9}$ Department of Wildlife and National Parks, Kuala Lumpur, Malaysia;

${ }^{10}$ Cognitive Ethology Laboratory, German Primate Center, Leibniz Institute for Primate Research, Goettingen, Germany; ${ }^{11}$ Gene Bank of Primates, German Primate Center, Leibniz Institute for Primate Research, Goettingen, Germany

Short title: Long-tailed macaque mitogenomics

*Correspondence to:

Rasmus Liedigk: rliedigk@gmx.de

Published in a modified version in BMC Genomics (2015) 16:222 


\section{ABSTRACT}

Long-tailed macaques (Macaca fascicularis) are an important model species in biomedical research, and reliable knowledge about their evolutionary history is essential for biomedical inferences. Ten subspecies have been recognized, of which most are restricted to small islands of Southeast Asia. In contrast, the common long-tailed macaque (M. f. fascicularis) is distributed over large parts of the Southeast Asian mainland and the Sundaland region. To shed more light on the phylogeny and phylogeography of $M$. $f$. fascicularis, we sequenced complete mitochondrial (mtDNA) genomes of 40 individuals from all over the taxon's range, either by classic PCR-amplification and Sanger sequencing or by DNA-capture and high-throughput sequencing. Within $M$. $f$. fascicularis, an initial split occurred 1.70 million years ago (Ma), separating haplotypes from mainland Southeast Asia, the Malay Peninsula and North Sumatra (Clade A), and haplotypes from Bangka, Java, Timor, Borneo, and the Philippines (Clade B). In Clade A, the three geographical populations appear as paraphyletic groups, while in Clade B local populations formed monophyletic clades with the exception of the Philippine individual which is nested within the Borneo clade. Further, in Clade B the branching pattern among main clades/lineages remained largely unresolved, most likely due to their rapid diversification 0.93-0.84 Ma. The application of complete mtDNA genomes yielded new insights into the evolutionary history of $M$. $f$. fascicularis by providing a more robust phylogeny and more reliable divergence age estimations which we used to discuss the phylogeography of the subspecies.

\section{Key words}

Southeast Asia, Sundaland, evolution, DNA, Sanger sequencing, highthroughput sequencing 


\section{INTRODUCTION}

Fossils indicate that macaques (genus Macaca) arose in Northeast Africa at around 7 million years ago (Ma) [Delson, 1975; Delson, 1980]. During their expansion into Asia in the Late Miocene, the genus diversified into various species groups and species that are defined by morphological, behavioral and molecular characters, and by their geographic distribution [Fooden, 1976; Delson, 1980; Groves, 2001; Tosi et al., 2003; Ziegler et al., 2007; Riley, 2010; Anandam et al., 2013; Zinner et al., 2013a]. Macaques represent one of the most successful extant primate radiations. They are found in over 20 Asian countries and in parts of northwestern Africa, and they represent the only cercopithecine genus in Asia. The colonized geographic range, from continents to islands, is unique among non-human primates [Abegg \& Thierry, 2002] and makes the genus Macaca an excellent example of adaptive radiation among primates [Riley, 2010].

Taxonomic affiliations of the various macaque species have been matter of debate for several decades [Fooden, 1976; Delson, 1980; Fooden, 1980; Groves, 2001; Tosi et al., 2000; Abegg \& Thierry, 2002; Tosi et al., 2002; Tosi et al., 2003; Ziegler et al., 2007]. According to current classifications the genus Macaca comprises 22 species, which are divided into seven species groups [Zinner et al., 2013a; Roos et al., 2014; Liedigk et al., in press], among them three monotypic species groups, (1) the M. sylvanus group, (2) the M. arctoides group and (3) the $M$. fascicularis group, and four polytypic groups, (4) the Sulawesi macaques group with six species, (5) the M. mulatta group with three species, (6) the M. sinica group with five species and (7) the M. silenus group with five species. M. arctoides is most likely of hybrid origin since it either clusters with the $M$. sinica group or the $M$. mulatta group depending on the investigated genetic marker, anatomical character or behavioral trait [Fooden, 1967; Fooden, 1976; Delson, 1980; Fooden, 1980; Deinard \& Smith, 2001; Tosi et al., 2003; Li et al. 2009; Perelman et al., 2011; Liedigk et al., in press]. The species composition of the $M$. fascicularis group has changed over time. Fooden [1976] and Delson [1980] included four species (M. mulatta, $M$. cyclopis, M. fuscata, M. fascicularis), but Groves [2001] separated M. mulatta, 
M. cyclopis and M. fuscata in their own species group, the M. mulatta group, but integrated $M$. arctoides in the $M$. fascicularis group. Zinner et al. [2013a] likewise recognized the members of the $M$. mulatta group as distinct species group and additionally excluded $M$. arctoides proposing a monotypic $M$. fascicularis group.

The long-tailed macaque ( $M$. fascicularis), as the only species of the $M$. fascicularis group [sensu Zinner et al., 2013a], has certainly the most discontinuous and beside rhesus macaques the largest distribution of all macaque species. Its range covers the southern part of the Southeast Asian mainland (Bangladesh, Myanmar, Thailand, Laos, Vietnam, Cambodia, peninsular Malaysia) as well as most of Sundaland (the islands of Borneo, Sumatra and Java, and adjacent islands) and beyond (central Indonesia, Philippines) (Fig. 1). On the basis of differences in pelage coloration and tail length ten subspecies are recognized [Fooden, 1980; Fooden, 1995; Fooden, 1997; Groves, 2001; Anandam et al., 2013; Zinner et al., 2013a; Roos et al., 2014; Roos \& Zinner et al., in press]. Three of them (M. f. aureus, M. $f$. fascicularis, M. f. philippinensis) have relatively large distributions, while all others (M. f. atriceps, M. f. condorensis, M. f. fuscus, M. f. karimondjawae, M. f. lasiae, M. f. tua, M. f. umbrosus) are restricted to small islands (Fig. 1). However, no genetic data is available yet to support this classification. So far, genetic studies have included only samples from M. f. fascicularis and M. $f$. philippinensis. Given the large and discontinuous range of $M$. $f$. fascicularis, it is not surprising that (genetic) variation within this subspecies is high [Harihara et al., 1988; Tosi et al., 2003; Smith et al.; 2007; Tosi \& Coke, 2007; Blancher et al., 2008; Kanthaswamy et al., 2008; Shiina et al., 2010; Berry et al., 2012; Kanthaswamy et al., 2013; Abdul-Latiff et al., 2014a; Abdul-Latiff et al., 2014b; Smith et al., 2014]. In fact, there is a deep genetic differentiation between M. $f$. fascicularis from the Asian mainland and Sundaland [Harihara et al., 1988; Tosi et al., 2003; Smith et al., 2007; Tosi \& Coke, 2007; Blancher et al., 2008; Kanthaswamy et al., 2008; Berry et al., 2012; Kanthaswamy et al., 2013; AbdulLatiff et al., 2014a; Abdul-Latiff et al., 2014b; Smith et al., 2014] and on Sumatra both Y-chromosomal lineages are found [Tosi \& Coke, 2007]. 


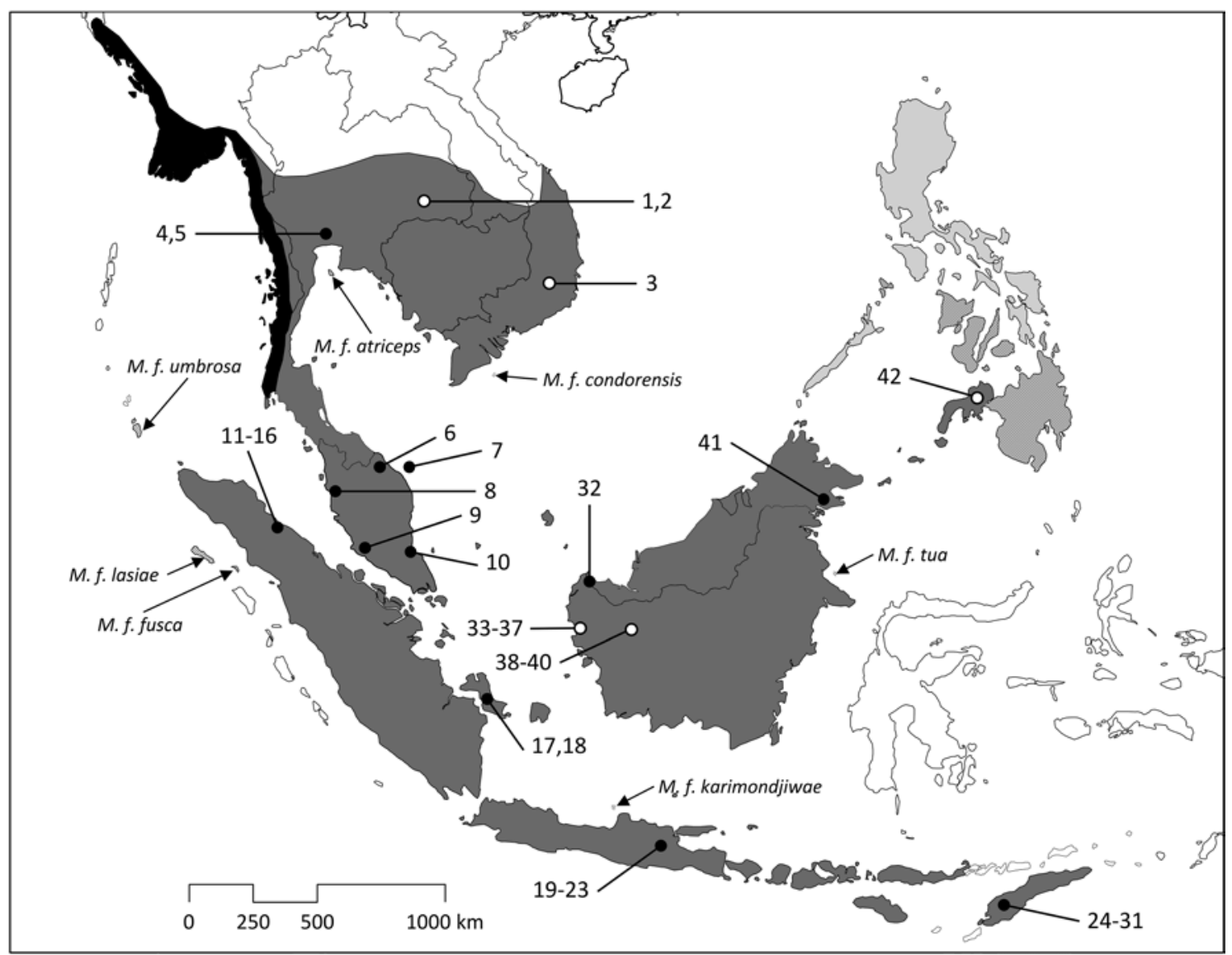

Fig. 1. Geographical distribution of long-tailed macaques (Macaca fascicularis) and sample collection sites. Species and subspecies distributions are depicted according to Fooden [1995] and adapted from Roos \& Zinner [in press]. The distribution of $M$. $f$. aureus, M. $f$. fascicularis and $M$. $f$. philippinensis is indicated by black, dark grey and light grey regions, respectively; the hatched region indicates the transition zone between latter two subspecies. Subspecies on small islands are named in the map. Open and filled circles indicate approximate and exact geographical origin of studied $M$. $f$. fascicularis individuals. ID numbers correspond to those in Fig. 2 and Supporting Information Table SI.

Besides the genetic variation among local long-tailed macaque populations, genetic evidence suggests that $M$. fascicularis on the Asian mainland was introgressed by rhesus macaques [Tosi et al., 2002; Tosi et al., 2003; Bonhomme et al., 2009; Stevison \& Kohn, 2009; Rovie-Ryan et al., 2013; Satkoski Trask et al., 2013a], and recent genome data indicate that around 30\% of the Asian mainland $M$. fascicularis genome is of rhesus macaque ( $M$. mulatta) origin [Yan et al., 2011]. This ancient hybridization (gene flow) most likely occurred unidirectional, from rhesus into long-tailed macaques and not vice versa [Tosi et al., 2002; Bonhomme et al., 2009; Stevison \& Kohn, 2009; Yan et al., 2011; Rovie-Ryan et al., 2013]. Even today, hybridization between 
both species occurs in a wide hybrid zone running from Vietnam, through Laos, Thailand, and probably into Myanmar [Fooden, 1997; Hamada et al., 2005]. Since the long-tailed macaque is an important model organism in biomedical research, reliable knowledge about their evolutionary history and genetic composition is essential for biomedical inferences (for an overview see [Haus et al., in press]).

The geographic origin of $M$. fascicularis and dispersal scenarios that led to its current distribution are still a matter of debate. Delson [1980] suggested that macaques entered Sundaland, probably in the Pliocene, during periods of low sea level and ancestral $M$. fascicularis became isolated there when rising sea levels and geological activity fragmented Sundaland. During the Pleistocene, $M$. fascicularis extended its range again [Delson, 1980; Fooden, 2006]. This largely corresponds to the observed higher level of nucleotide diversity found in longtailed macaque populations from Sundaland compared to the populations from the Asian mainland and Malay Peninsula [Smith et al., 2007; Berry et al., 2012; Kanthaswamy et al., 2013; Rovie-Ryan et al., 2013; Smith et al., 2014]. This scenario is also in agreement with the fact that the earliest fossils of $M$. fascicularis, or at least those of a close relative, were found on Java [Delson, 1980; Aimi \& Aziz, 1985; Fooden, 2006]. Currently, the species is also found on islands that were never connected to the Asian mainland or Sundaland, including islands east of the Wallace line (e.g., Lombok, Sumbawa, Flores, Timor) and the Philippines. Accordingly, it was assumed that humans introduced $M$. fascicularis to the islands east of the Wallace line ca. 4,000 year ago [Fooden, 2006], while the Philippines were most likely naturally colonized during two independent migration events [Smith et al., 2014]. The species' survival in other areas where it has been introduced by humans (e.g., Hong Kong, Taiwan, Papua New Guinea, New Britain, and various Pacific islands) indicates its considerable ecological plasticity. Long-tailed macaques are highly adaptable to riverine and coastal environments such as mangrove and gallery forests [Fittinghoff \& Lindburg, 1980; Wheatley, 1980; Harcourt \& Meijaard, 2013]. M. fascicularis primarily feeds on fruits and seeds [Yeager, 1996], but as indicated by one of its common names, crab-eating macaque, it also includes 
crabs, shrimps, clams and fishes in its diet [Stewart et al., 2008], and is able to swim and even to dive [Son, 2003; Gumert \& Malaivijitnond, 2012]. Hence, it is likely that long-tailed macaques were able to cross short distances between islands by swimming.

The Southeast Asian mainland and the Sundaland region experienced dramatic geographical and environmental changes that repeatedly occurred during the last two million years [Voris, 2000; Meijaard, 2003; Bird et al., 2005; Woodruff, 2010]. A recent biogeographic review for the region [de Bruyn et al., in press] identified three predominant sea-level scenarios for the middle to late Pleistocene in this region. The most frequently recurring scenario $( \pm 55 \%$ of last million years) is of periods with sea-levels $40-50 \mathrm{~m}$ below current levels, leaving around half of the current Sunda Shelf emergent, and evergreen rainforests extended across much of the region. The second most frequent scenario $( \pm 37 \%$ of last million years) is of periods with very low sea levels $(>100 \mathrm{~m}$ below current levels), such as during the Last Glacial Maximum (LGM). Seasonal vegetation was very widespread, though may not have formed a continuous north to south corridor for every glacial maximum, and the exposed and mostly vegetation free sandy soils of the shelf may have acted as a significant barrier to dispersal [Slik et al., 2011]. The degree of climatic seasonality may have varied between glacial maxima, suggested by the occurrence of certain mammalian fossils in Java. For example, faunas from the penultimate and older glacials included many large mammals, such as Rhinoceros unicornis, Stegodon trigonocephalus, Hippopotamus sivalensis, and Hyaena brevirostris requiring open woodland [Louys \& Meijaard, 2010; van den Bergh et al., 2011]. The third climatic scenario is represented by the present day situation, with high sea levels and evergreen rainforests extending from the Isthmus of Kra to West Java, including Borneo. This situation, however, prevailed for just $8 \%$ of the last million years, emphasizing the 'refugial' nature of present day Southeast Asian rainforests and fauna [Cannon et al., 2009]. These glacial events are thought to have triggered repeated biotic range expansions between long-tailed macaques in Sumatra, the Thai-Malay Peninsula, Borneo and Java. The respective species would likely have dispersed between these areas through coastal 
forests and along rivers at times of low sea-level, swimming across rivers or rafting on floating vegetation. Times of high sea-levels would have resulted in vicariance.

The objective of this study is to shed more light on the phylogeny and phylogeography of $M$. $f$. fascicularis, the most widespread subspecies of the long-tailed macaques, occurring on the Southeast Asian mainland and Sundaland islands, including parts of the Philippines and east as far as Timor. Therefore, we generated complete mitochondrial (mtDNA) genomes from 40 long-tailed macaque individuals either by traditional polymerase chain reaction (PCR) amplification followed by Sanger sequencing or by DNA-capture and high-throughput sequencing. We expect that the analysis of complete mtDNA genomes provide a better resolution of phylogenetic relationships among lineages than only single mtDNA fragments.

\section{METHODS}

\section{Sample collection}

We collected samples from 41 long-tailed macaque individuals originating from 16 sites throughout the species' range in Southeast Asia and Sundaland, and from the introduced population on Mauritius (Fig. 1, Supporting Information Table SI). Thirty-one of our samples (sample IDs: 4, 5, 11-31, 33-40) derived from museum specimens housed in the Bavarian State Collection of Zoology (ZSM) in Munich, Germany. Respective specimens were collected between 1904 and 1949. Dried muscle tissue attached to the skeleton was taken with sterilized scalpels and tweezers, and gloves and masks were worn during sample collection to avoid contamination. Museum samples were stored dry in tubes or plastic envelopes. Additionally, we included seven fresh fecal samples, stored in $90 \%$ ethanol, which were collected during field surveys (IDs: 6-10, 32, 41). We further obtained high-quality DNA extracted from blood samples from two individuals from Covance Inc. (Muenster, Germany) and one individual from the German Primate Center (DPZ, Goettingen, Germany) which originated from Vietnam (ID: 3 ; the mtDNA genome of this specimen is already published 
[Liedigk et al., in press], but was herein used as bait for DNA capture) and the Philippines (ID: 42), and Mauritius (ID: 43), respectively. For all samples, we tried to obtain information about the exact geographic provenance, but this was not always possible. While for all fecal samples, GPS coordinates were recorded, information about the exact origin of the samples from Vietnam, the Philippines and Mauritius is not available. Likewise, we were not able to identify the exact provenance of five Bornean samples (IDs: 33-37, derived from "west coast Borneo"), while for all other museum samples the exact origin could be determined. Thus, 39 samples can be geographically clearly assigned to M. $f$. fascicularis (IDs: 3-41). The individual from Mauritius (ID: 43) most like refers also to $M$. f. fascicularis because it is believed that this introduced population originated from Sumatra [Tosi \& Coke, 2007; Satkoski Trask et al., 2013b], while the individual from the Philippines (ID: 42) could be either $M$. $f$. philippinensis or M. f. fascicularis (due to its haplotype it is most likely M. $f$. fascicularis). For detailed sample information see Supporting Information Table SI.

All research in this project complied with protocols approved by DPZ in Germany and the Department of Wildlife and National Parks in Malaysia, and adhered to the legal requirements of the countries in which the research was conducted. The study was carried out in compliance with respective animal care regulations and the principles of the American Society of Primatologists for the ethical treatment of non-human primates.

\section{DNA extraction}

For the extraction of total genomic DNA we used two different methods. First, we applied a kit-based method using the First-DNA All Tissue kit (Genlal). All fecal and five of the museum samples (IDs: 11, 14, 20, 31, 38) were extracted with this method following respective protocols provided by the company. To avoid and check for cross-sample contamination, all working steps were carried out in separate laboratories and under Captair Bio PCR cabinets (Erlab), gloves and masks were permanently worn, and negative extraction controls were routinely conducted. Further, samples were treated one by one, 
and workbenches were decontaminated with UV light before and after every extraction. After extraction, DNA concentration was measured on a NanoDrop ND-1000 spectrophotometer and samples were stored at $-20^{\circ} \mathrm{C}$ until further processing. Secondly, 28 museum samples (IDs: 4, 5, 12, 13, 15-31, 33-37, 39, 40) were extracted in a special ancient DNA laboratory applying a protocol for nondestructive DNA extraction [Rohland et al., 2004, 2010] with slight modifications [Haus et al., 2013]. All working steps were carried out in Thermo Scientific Safe 2020 biological safety cabinets. For each step (sample preparation, DNA extraction) different cabinets were used, and before and after each sample, cabinets were cleaned with DNA decontamination solution and treated with UV light for at least $30 \mathrm{~min}$. Concentration of extracted DNAs was measured on a Qubit 2.0 fluorometer and DNA samples were frozen at $-20^{\circ} \mathrm{C}$ until further processing. For comparative reasons, two museum samples (IDs: $20,31)$ were extracted with both methods.

\section{DNA amplification and Sanger sequencing}

We generated complete mtDNA genomes from the high-quality samples from the Philippines and Mauritius as well as from three of the fecal samples (IDs: 7, 32, 41) and five of the museum samples (IDs: 11, 14, 20, 31, 38) by traditional PCR amplification followed by Sanger sequencing. All working steps (PCR setup, gel electrophoresis, PCR product purification, sequencing) were conducted in separate laboratories and under Captair Bio PCR cabinets to prevent cross-sample contamination. Further, negative PCR controls (without template DNA) were routinely conducted. To minimize the risk of amplifying nuclear mitochondrial-like sequences (numts) for the two high-quality DNA samples, we produced two overlapping long-range PCR products $(8 \mathrm{~kb}$ and 10 $\mathrm{kb}$ ) followed by nested PCRs with product sizes of 1.0-1.2 kb applying methods described in detail elsewhere [Liedigk et al., in press]. Since DNA extracted from fecal and museum samples is usually degraded, the complete mtDNA genome from these samples was directly amplified via 21 100-300 bp overlapping fragments and not first via two long-range PCRs. For the amplification, we used the same primers as employed above for the nested 
PCRs. PCR conditions were the same as for the nested PCRs above, but sometimes the number of cycles was increased to 60 . As template, we added 10-50 ng DNA to the reaction. PCR performance and product sizes were checked on $1 \%$ agarose gels, and after purification, PCR products were sequenced on an $A B I 3130 x L$ sequencer using the BigDye Terminator Cycle Sequencing kit (Applied Biosystems) and both amplification primers. Information on primers and PCR conditions is available upon request. Sequences were checked with 4Peaks 1.7.1 (mekentosj.com) and mtDNA genomes were assembled with SeaView 4.4.0 [Gouy et al., 2010]. Annotation was performed with DOGMA [Wyman et al., 2004] and manually verified.

\section{DNA-capture and high-throughput sequencing}

Complete mtDNA genomes from 28 museum (IDs: 4, 5, 12, 13, 15-31, 33$37,39,40$ ) and four fecal samples (IDs: 6, 8-10) were generated using a DNAcapture approach followed by high-throughput sequencing according to Maricic et al. [2010] with slight modifications (see below) to adapt the workflow to the Ion PGM sequencing system (Ion Torrent). To prevent contamination, all working steps were carried out in dedicated ancient DNA and/or special highthroughput sequencing laboratories, and various negative controls were applied. After DNA extraction and concentration measurement, barcoded sequencing libraries were established using the Ion Plus Fragment Library kit and the Ion Xpress Barcode Adapters. Adapter ligation and the subsequent amplification of the samples were performed according to the protocol for lon Xpress Plus gDNA Fragment Library Preparation. Afterwards, we pooled the adapter-ligated and amplified libraries in equal concentrations to a total of $2 \mu \mathrm{g}$. As bait we used mtDNA genomes of each one long-tailed macaque individual from Vietnam (ID: 3 ) and Mauritius (ID: 43). The respective complete mtDNA genomes were amplified via two overlapping PCR products (see above). Afterwards, we sheared the PCR products to an average of ca. 1,000 bp fragments with a Bioruptor Pico. We diluted $1.5 \mu \mathrm{g}$ of PCR product to a volume of $150 \mu \mathrm{l}$, split the sample into three $(50 \mu \mathrm{l}$ each) and sonicated each six times with 10 seconds "ON" and 90 seconds "OFF". One $\mu$ of the sheared PCR 
product was size-checked on the Agilent 2100 Bioanalyzer with the high sensitivity DNA kit. Fragments were subsequently end-repaired, biotinylated by ligating the Bio-T/B adapter [Maricic et al., 2010] and immobilized on streptavidin-coated beads. Bait and the pooled single-stranded libraries were combined and four phosphorylated blocking oligos (BO1.P1.F: CCACTACGCCTCCGCTTTCCTCTCTATGGGCAGTCGGTGAT-phosphate, BO2.P1.R: ATCACCGACTGCCCATAGAGAGGAAAGCGGAGGCGTAGTGGphosphate, BO3.A.F: CCATCTCATCCCTGCGTGTCTCCGACTCAGphosphate, BO4.A.R: CTGAGTCGGAGACACGCAGGGATGAGATGGphosphate) were added. After $48 \mathrm{~h}$ of hybridization at $65^{\circ} \mathrm{C}$, library molecules that did not hybridize were washed out and the enriched library pool was eluted. Subsequently, the concentration of the enriched library pool was measured by qPCR (Ion Library Quantitation Kit) and sequenced on the Ion PGM sequencer using a 316v2 or 318v2 chip and the Ion PGM Sequencing 400 Kit protocol. The raw sequencing reads were quality-filtered, and adapters and barcodes were trimmed with the PGM Torrent Suite Software 4.2. The extracted reads were initially assembled by running the Newbler program (GS Reference Mapper) of the 454 Sequencing System Software 2.5 from command line with standard parameters. The mtDNA genome of the Vietnamese $M$. fascicularis individual (ID: 3 ) was used as reference. Batch processing was done by custom Perl scripts. The resulting contigs, typically ranging from 1 to 4 sequences per mtDNA genome, were manually assembled into genomes with SeaView and annotated with DOGMA. All gaps between contigs could be closed by combining the results from multiple sequencing runs.

\section{Statistical analyses}

For phylogenetic reconstructions, we expanded our dataset with additional mtDNA genome sequences from macaque and non-macaque taxa. The dataset comprised $60 \mathrm{mtDNA}$ genomes including $43 \mathrm{M}$. fascicularis individuals, at least one representative of the other six macaque species groups (2 M. sylvanus, 1 M. arctoides, $3 \mathrm{M}$. mulatta, $2 \mathrm{M}$. thibetana, $1 \mathrm{M}$. tonkeana, $1 \mathrm{M}$. silenus) and various outgroup taxa (1 Theropithecus gelada, 1 Papio hamadryas, 1 
Chlorocebus pygerythrus, 1 Colobus guereza, 1 Pongo abelii, 1 Pan troglodytes, 1 Homo sapiens). For detailed sample information and Genbank accession numbers see Supporting Information Table SI.

Sequences were aligned with Muscle 3.7 [Edgar, 2004] as implemented in SeaView and manually corrected. Indels and poorly align positions were removed with Gblocks 0.91b [Castresana, 2000] using standard settings. Identical sequences were subsequently excluded (IDs: $21=23,27=28=31$, $33=34$ ), resulting in a final dataset of 56 unique mtDNA genome haplotypes. For Maximum Likelihood (ML) and Bayesian tree reconstructions, we applied the programs RAxML 0.93 [Stamatakis, 2006] and MrBayes 3.1.2 [Huelsenbeck et al., 2001; Ronquist \& Huelsenbeck 2003], respectively. ML calculations in RAxML were run with the CAT-GTR model and 1,000 bootstrapping replications. For Bayesian tree reconstructions in MrBayes, we conducted four Markov Chain Monte Carlo (MCMC) runs with a default temperature of 0.2 and the $\mathrm{TrN}+\mathrm{l}+\mathrm{G}$ model as selected as best-fit model in jModeltest 2.1 [Posada, 2009] under the Bayesian information criterion (BIC) and the Decision Theory Performance-based Selection (DT). All repetitions were run for 1 million generations with tree and parameter sampling setting in every 100 generations. The first $25 \%$ of samples were discarded as burn-in, resulting in 75,001 trees per run. The adequacy of the burn-in and convergence of all parameters was assessed via the uncorrected potential scale reduction factor (PSRF) [Gelman \& Rubin, 1992] as calculated by MrBayes and by visual inspection of the trace of the parameters across generations using TRACER 1.5 [Rambaut \& Drummond, 2007]. To check whether posterior clade probabilities were also converging, AWTY [Nylander et al., 2008] was applied. Posterior probabilities for each split and a phylogram with mean branch lengths were calculated from the posterior density of trees.

Divergence ages from the dataset were estimated with BEAST 1.6.1 [Drummond \& Rambaut, 2007] applying a Bayesian MCMC method with a relaxed molecular clock approach [Drummond et al., 2006]. A relaxed lognormal model of lineage variation and a Birth-Death Process prior for branching rates was assumed. The following five fossil-based calibration points were used with 
a normal distribution prior for respective nodes: (1) the Homo - Pan split 6.5 Ma with a $95 \%$ credibility interval $(\mathrm{Cl})$ of $0.5 \mathrm{Ma}$ [Vignaud et al., 2002; Brunet et al., 2005; Lebatard et al., 2008], (2) the split between Pongo and the Homo-Pan lineage at $14 \mathrm{Ma}(95 \% \mathrm{Cl}: 1.0 \mathrm{Ma})$ [Kelly, 2002], (3) the divergence of Theropithecus and Papio $5 \mathrm{Ma}(95 \% \mathrm{Cl}: 1.5 \mathrm{Ma})$ [Leakey, 1993; Delson, 2000], (4) the M. sylvanus - M. mulatta split at $5.5 \mathrm{Ma}(95 \% \mathrm{Cl}: 1.0 \mathrm{Ma})$ [Delson, 2000; Alba et al., 2014] and (5) the divergence of hominids and cercopithecids at 27.5 Ma (95\% Cl: 3.5) [Zalmout et al., 2010; Pozzi et al., 2011]. Four replicates were run in BEAST for 25 million generations with tree and parameter sampling occurring every 100 generations. TRACER was used to assess the adequacy of a $10 \%$ burn-in and the convergence of all parameters via visual inspection of the trace of the parameter across generations. Sampling distributions were combined (25\% burn-in) using the software LogCombiner 1.6.1. A consensus chronogram with node height distribution was generated and visualized with FigTree 1.3.1 [Rambaut, 2008].

\section{RESULTS}

We generated 42 complete mtDNA genome sequences from $40 \mathrm{M}$. fascicularis individuals, either by classic PCR followed by Sanger sequencing (10 individuals) or by DNA-capture and high-throughput sequencing (32 individuals). For two museum samples (IDs: 20, 31) both methods were applied which yielded identical sequences. For mtDNA genomes that were captured and sequenced on the lon PGM sequencing platform we obtained an average of $97,583(12,599-230,683)$ trimmed reads with an average read length of 96 bp, resulting in an average 285-fold coverage. Sequences in the overlapping parts were identical and all protein-coding genes were correctly translated without any premature stop codons, indicating that no numts are present in our dataset. All newly generated mtDNA genomes had a length of 16,561 to 16,567 bp, and consisted of 22 transfer RNA genes, 2 ribosomal RNA genes, 13 protein coding genes and the control region. 
The original alignment for phylogenetic analysis with 60 primate sequences had a length of $16,874 \mathrm{bp}$, but was reduced to $15,868 \mathrm{bp}$ after indels and poorly aligned positions were removed. Some individuals shared the same haplotype (IDs: $21=23,27=28=31,33=34$ ). These were excluded resulting in a final alignment of 56 unique primate mtDNA genome haplotypes. Phylogenetic trees as obtained from $M L$ and Bayesian analyses are mainly identical and most nodes are strongly supported (ML bootstrap values: $>95 \%$, Bayesian posterior probabilities: 1.0) (Fig. 2, Supporting Information Fig. SI). According to estimated divergence ages, Hominidae and Cercopithecidae separated 28.60 Ma (for estimates and their $95 \% \mathrm{Cls}$ see Supporting Information Table SII). Among hominids, Pongo diverged from the Homo + Pan clade $13.82 \mathrm{Ma}$, while the latter split 6.32 Ma. Among cercopithecids, Colobus diverged first, 19.89 $\mathrm{Ma}$, and Chlorocebus separated from papionins $12.81 \mathrm{Ma}$. In the Papionini clade, Theropithecus + Papio diverged from the macaques $10.90 \mathrm{Ma}$, while the former two genera split 4.77 Ma. Within macaques, M. sylvanus (from northern Africa) branched off first, 6.10 Ma. The remaining, solely Asian macaque species, diverged into two clades $5.49 \mathrm{Ma}$, one comprising $M$. silenus and $M$. tonkeana, and the other $M$. thibetana, $M$. arctoides, $M$. mulatta and $M$. fascicularis. In the former clade, $M$. silenus and M. tonkeana separated 3.70 $\mathrm{Ma}$, while in the latter clade M. thibetana split off first, $4.16 \mathrm{Ma}$, followed by $M$. fascicularis $3.42 \mathrm{Ma}$, before finally M. mulatta and M. arctoides diverged 3.02 Ma. Within $M$. fascicularis, an initial split occurred $1.70 \mathrm{Ma}$, separating haplotypes from mainland Southeast Asia, Peninsula Malaysia and Sumatra (Clade A), and individuals from Borneo, Java, Bangka, Timor, the Philippines and Mauritius (Clade B). In Clade A, individuals from mainland Southeast Asia, Peninsula Malaysia and Sumatra do not form reciprocally monophyletic clades. Splitting events within Clade A occurred 0.96-0.02 Ma. In Clade B, individuals from different geographic regions form monophyletic clades or represent distinct lineages. The only exception is the Borneo clade which comprises also the individual from the Philippines (ID: 42). In clade B, the branching pattern among main clades/lineages remains unresolved indicating a diversification within a short time period. In fact, this radiation occurred between $0.93 \mathrm{Ma}$ and $0.84 \mathrm{Ma}$, 
thus in less than 100,000 years. Individuals from Bangka (IDs: 17, 18), an island east of Sumatra (Fig. 1), form a monophyletic clade and cluster together with the Borneo/Philippines clade. Both of these clades shared a common ancestor until $0.61 \mathrm{Ma}$. The Philippine individual is nested within the Borneo clade and specifically clusters with an individual from Sabah (ID: 41); they diverged from each other $0.21 \mathrm{Ma}$.

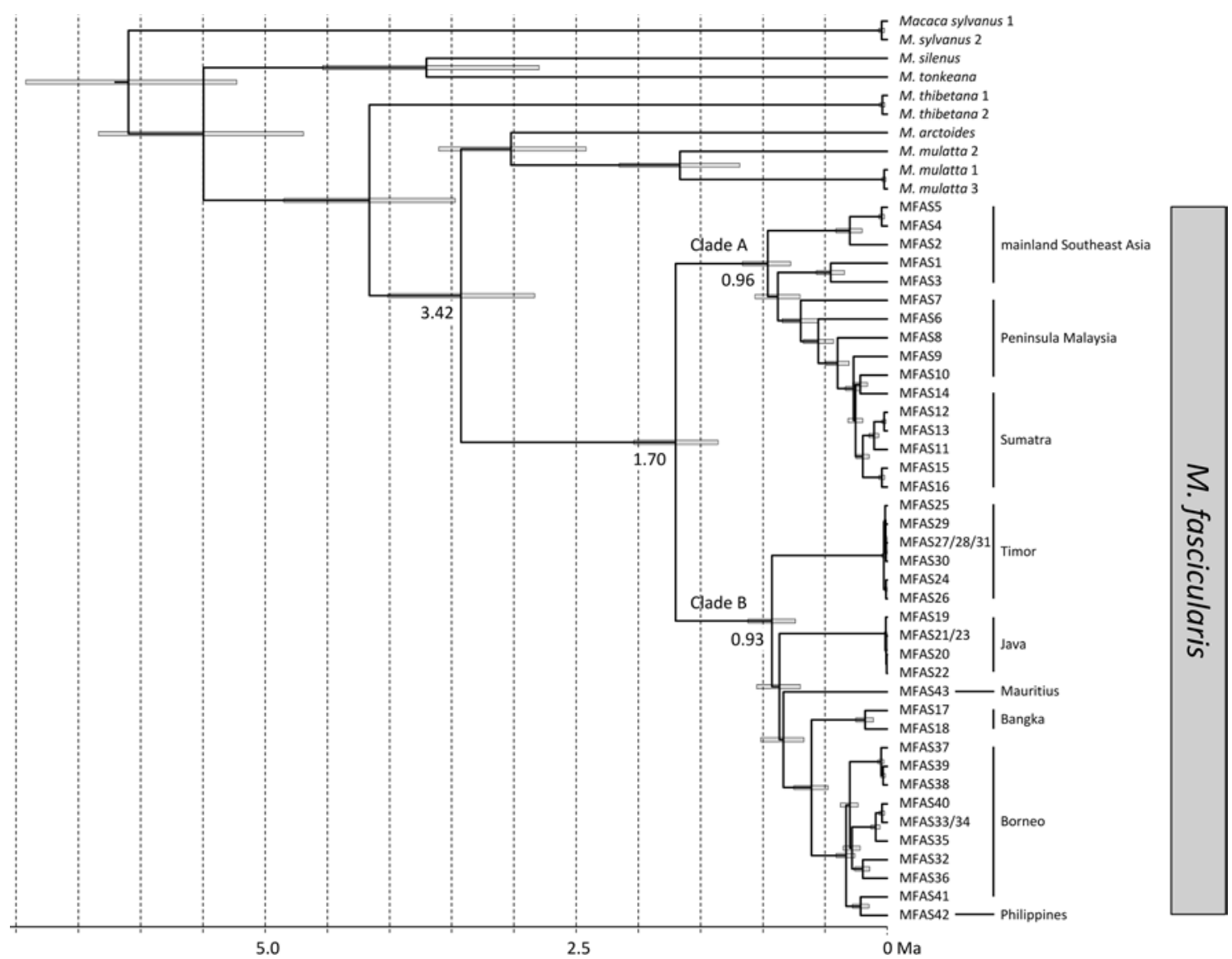

Fig. 2. Ultrametric tree showing phylogenetic relationships and divergence ages among macaques as calculated from complete mtDNA genome sequences. Grey bars indicate $95 \%$ credibility intervals of divergence times and the time scale below shows million years before present. Numbers correspond to IDs in Fig. 1 and Supporting Information Table SI. For detailed information of divergence ages and statistical node support see Supporting Information Table SII and Fig. SI, respectively.

\section{DISCUSSION}

By applying different methods, classic PCR amplification followed by Sanger sequencing and DNA-capture with subsequent high-throughput 
sequencing, we successfully obtained complete mtDNA genome data from 40 M. fascicularis individuals. Both methods have proven to be useful to gain such data, but the DNA-capture and high-throughput sequencing approach is less cost and time intensive [Maricic et al., 2010; Gunnarsdóttir et al., 2011; Guschanski et al., 2013]. Moreover, DNA extracted from museum material and feces is normally highly degraded. Fortunately, some of our museum and fecal samples contained DNA in sufficient quality so that the complete mtDNA genome could be amplified via just 21 overlapping PCRs. Usually, due to the high grade of DNA degradation, generating complete mtDNA genomes would require a large number of PCR amplifications. In contrast, DNA capture does not need a certain DNA fragment size, because any size of DNA fragment can be captured and subsequently sequenced. However, with decreasing lengths of generated sequences, an appropriate reference sequence from a phylogenetically close taxon is required for a reliable assembly, particularly for the variable control region.

Our results concerning the phylogenetic relationships among macaque and non-macaque taxa and estimated divergence ages are largely in line with previous molecular studies [Morales \& Melnick, 1998; Tosi et al., 2000; Deinard \& Smith, 2001; Tosi et al., 2002; Tosi et al., 2003; Tosi \& Coke, 2007; Ziegler et al., 2007; Li et al., 2009; Perelman et al., 2011; Springer et al., 2012; Finstermeier et al., 2013; Pozzi et al., 2014; Liedigk et al., in press]. For the phylogenetic relationships among $M$. fascicularis haplotypes, we gain higher statistical support for most nodes in our tree, compared to earlier mtDNA studies which mainly used only fragments of the mtDNA genome [Tosi et al., 2000; Tosi et al., 2002; Tosi et al., 2003; Smith et al., 2007; Tosi \& Coke, 2007; Blancher et al., 2008; Shiina et al., 2010]. Nevertheless, some nodes in our study are still missing significant statistical support, thus leaving some phylogenetic relationships, mainly those between populations from Timor, Java, Mauritius and Bangka/Borneo/Philippines, unresolved. Such results are common when clades or lineages diverge within a short time period [Roos et al., 2011; Liedigk et al., 2012; Guschanski et al., 2013; Zinner et al., 2013b; Carbone et al., in press; Liedigk et al., in press]. In contrast to Tosi \& Coke 
[2007] who found Sumatran individuals to be part of the Sundaland clade, the Sumatran individuals which we used are nested within the Asian mainland clade. A possible explanation for these contradictory results is most likely the different origin of studied individuals, with the South Sumatran origin samples of Tosi \& Coke [2007] clustering with Sundaland sequences and our North Sumatran samples clustering with the mainland clade. The presence of representatives of both major M. $f$. fascicularis mtDNA clades on Sumatra is further supported by the likewise presence of both $Y$ chromosomal haplogroups on the island [Tosi \& Coke, 2007].

Since mtDNA is only inherited via the maternal line and macaques live mainly in female philopatric societies [Pusey \& Packer, 1987; de Ruiter \& Geffen 1998], mtDNA data can be utilized to reveal insights into genetic differences among regional populations and to trace their phylogeographic history [Avise, 2004]. According to our phylogeny and estimated divergence ages, M. f. fascicularis initially split into an Asian mainland and a Sundaland clade $1.70 \mathrm{Ma}$, and both clades are found on Sumatra (according to our data and [Tosi \& Coke, 2007]). Possible explanations are (1) Sumatra is the place of origin of M. f. fascicularis, (2) Sumatra is the place of origin of only Sundaland M. f. fascicularis, while long-tailed macaques from the mainland invaded the island later, or (3) that long-tailed macaques on Sumatra became extinct and the island was later re-colonized from the mainland and other Sundaland islands. The hypothesis that Sumatra is the place of M. f. fascicularis origin is supported by the observed high mtDNA diversity found on the island compared to other regions where the subspecies occurs [e.g., Smith et al., 2014]. It has been hypothesized that the northern part of Sumatra may have been separated from southern Sumatra during much of the Pleistocene due to presence of a sea strait that separated Sumatra into two islands [van Bemmelen, 1970; Meijaard, 2003; Meijaard \& Groves, 2004]. This would explain the divergence of northern and southern Sumatra populations. The northern Sumatra population became reconnected to the Malay Peninsula and further to the Asian mainland via existing land bridges. The southern Sumatran population on the other hand remained separated from the Malay Peninsula and the Asian mainland, but 
dispersed successively into Borneo, Java and further to the east. However, not in support of this hypothesis is the paraphyly of haplotypes from the mainland and Malay Peninsula, and the respective branching pattern among them and the Sumatra haplotypes, which suggests that the northern Sumatra population originated on the mainland. We further note that the geological evidence of a sea strait between northern and southern Sumatra remains tentative and that other factors, such as repeated volcanic eruptions in the Toba complex, could have also played a role [Nater et al., 2011; Louys, 2012; Wilting et al., 2012], even leading to the extinction of long-tailed macaques on Sumatra. To test whether Sumatra or any other island, e.g., Java [Delson, 1980; Smith et al., 2007 ] is the place of origin of Sundaland $M$. f. fascicularis needs further investigations and, particularly, should include data of $M$. $f$. fascicularis from southern Sumatra. Cercopithecid fossils from Sumatra are rather scarce compared to those from Java, and are limited to the Late Pleistocene and Holocene. They consist mainly of teeth or fragments that can hardly be assigned to particular species [Hooijer, 1962]. The paucity of clear macaquelike fossils, therefore, does not necessarily exclude Sumatra as potential origin of $M$. f. fascicularis.

As in previous studies [Tosi et al., 2003; Tosi \& Coke, 2007; Smith et al., 2014], we found long-tailed macaques from the Philippines clustering within the Borneo clade. Since the Bornean individual, which is most closely related to the Philippine specimens, is from the furthest east of Borneo (Sabah, Tawau Hill Park), this branching pattern fosters the previously proposed hypothesis of a colonization of the Philippines via Borneo [Brandon-Jones, 1996; Abegg \& Thierry, 2002; Smith et al., 2014]. Within the last million years, the Philippines have never been connected to the Southeast Asian mainland or Borneo via a continuous land bridge [Esselstyn et al., 2004]. Volcanic activity and uplift of oceanic crust resulted in numerous small islets between Borneo and the Philippines, which were most likely always isolated by sea channels [Heaney, 1985; Heaney, 1986; Heaney, 1991; Hall, 1998; Hall, 2002]. One possible exception is the island of Palawan which has been considered as dry land connection to Borneo during sea-level lows in the Late Pleistocene [Heaney, 
1986]. Although it is not clear whether this connection has been continuous or if seawater channels interrupted it [Rohling et al., 1998; Voris, 2000], one can assume that the gap between Borneo and Palawan was relatively narrow [Esselstyn et al., 2004]. Given that long-tailed macaques are highly adaptable to riverine and coastal environments as mangrove forests and riversides [Fittinghoff \& Lindburg, 1980; Wheatley, 1980], the previously proposed Philippine colonization hypothesis [Abegg \& Thierry, 2002; Brandon-Jones, 1996] via Palawan and appending islets seems plausible (stepping-stone colonization). A recent study in fact suggests that there may have been at least two dispersal events from Borneo into the Philippines, first one via Palawan resulting in M. f. philippinensis in the north of the Philippine Archipelago, and a later one via the Sulu Archipelago that resulted in M. f. fascicularis in the south [Smith et al., 2014], the taxon which was included in the present study.

One noteworthy outcome from our study is the early divergence of a monophyletic Timor clade within the Sundaland clade (Fig. 2). It appears that this clade diverged some $0.93(1.12-0.74) \mathrm{Ma}$ from the other Sundaland lineages. This finding is supported by analysis of blood protein polymorphisms from samples across the Indonesian and Timor island arc which indicated that populations east of the Wallace Line (Lombok and Sumbawa) have greatly differentiated from those to the west [Kawamoto et al., 1984]. Our mtDNAbased estimate, however, significantly predates the earliest finds of macaques in Timor's archaeological record, which appear at the same time, i.e. a few thousand years ago, as the first evidence of pottery and domesticated pig in one site (Uai Bobo 1 and 2), indicating human translocations [Glover, 1986]. Similarly, on Flores, an island further west, but still east of the Wallace Line, long-tailed macaques only appear in the archaeological record around 7,000 years ago [van den Bergh et al., 2009]. It is unclear what underlies the apparent major discrepancy between the present phylogenetic analysis and the zooarchaeological record, but an introduction by humans as proposed [Fooden, 2006] seems unlikely, although the possibility remains that the detected Timor haplotypes originated from somewhere else in Sundaland, a place that was not sampled in our study. 


\section{CONCLUSIONS}

Both applied laboratory methods have proven to be powerful to generate complete mtDNA genome data, with the DNA-capture and high-throughput sequencing approach as the most promising and only option to obtain such data from highly degraded DNA, in time and with relatively low costs. Nevertheless, appropriate reference sequences from phylogenetically close taxa are essential for reliable assemblies, particularly for the variable control region.

Our study provides new insights into the evolutionary and phylogeographic history of M. f. fascicularis, most prominent the confirmed mainland clade in North Sumatra and the clearly distinct and old Timor clade. However, to identify the origin of long-tailed macaques and their dispersal routes leading to their current distribution, to assess their full genetic diversity and to explore to which extent secondary gene flow occurred between local populations, it is fundamental to include further $M$. $f$. fascicularis populations from throughout their range into the studies. In these studies both, mitochondrial and a large number of nuclear loci, should be analyzed. Moreover, to fully understand the evolutionary and phylogeographic history of the species, the other subspecies of $M$. fascicularis should be incorporated in such studies as well.

\section{ACKNOWLEDGEMENTS}

We are grateful to the Bavarian State Collection of Zoology, Covance Inc. and the German Primate Center for providing valuable long-tailed macaque samples, as well as to the Universiti Kebangsaan Malaysia, Department of Wildlife and National Parks (PERHILITAN), Sarawak Forest Department, Sabah Wildlife Department and Sabak Parks for sharing fecal samples. This research was financially supported by the German Primate Center and by grants to BM Md-Zain (FRGS/1/2012/STWN10/UKM/02/3, DLP-2013-006, ERGS/1/2013/STWN10/UKM/02/1). We further thank Christiane Schwarz, Nico Westphal and Jens Gruber for support during laboratory work and statistical analysis. 


\section{REFERENCES}

Abdul-Latiff MAB, Ruslin F, Fui VV, et al. 2014a. Phylogenetic relationships of Malaysia's long-tailed macaques, Macaca fascicularis, based on cytochrome $b$ sequences. Zookeys 407:121-139.

Abdul-Latiff MAB, Ruslin F, Faiq H, et al. 2014b. Continental monophyly and molecular divergence of Peninsular Malaysia's Macaca fascicularis fascicularis. BioMed Research International 2014:897682.

Abegg C, Thierry B. 2002. Macaca evolution and dispersal in insular south-east Asia. Biological Journal of the Linnean Society 75:555-576.

Aimi M, Aziz F. 1985. Vertebrate fossils from the Sangiran Dome, Mojokerto, Trinil and Sambungmacam areas. In: Watanabe N, Kadar D, editors. Quaternary geology of the hominid fossil bearing formations in Java. Report of the Indonesia-Japan Joint Research Project CTA-41, 19761979. Bandung: Geological Research \& Development Centre. p 155-198.

Alba DM, Delson E, Carnevale G, et al. 2014. First joint record of Mesopithecus and cf. Macaca in the Miocene of Europe. Journal of Human Evolution 67:1-18.

Anandam MV, Bennett EL, Davenport TRB, et al. 2013 Family Cercopithecidae (Old World Monkeys) - Species accounts for Cercopithecidae. In: Mittermeier RA, Rylands AB, Wilson DE, editors. Handbook of the mammals of the world. Volume 3, Primates. Barcelona: Lynx Edicions. $p$ 628-753.

Avise JC. 2004. Molecular markers, natural history, and evolution. Sunderland: Sinauer Associates. $684 \mathrm{p}$.

Berry NJ, Marzetta F, Towers GJ, Rose NJ. 2012. Diversity of TRIM5a and TRIMCyp sequences in cynomolgus macaques from different geographical origins. Immunogenetics 64:267-278.

Bird MI, Taylor D, Hunt C. 2005. Palaeoenvironments of insular Southeast Asia during the Last Glacial Period: A savanna corridor in Sundaland? Quaternary Science Reviews 24:2228-2242.

Blancher A, Bonhomme M, Crouau-Roy B, et al. 2008. Mitochondrial DNA 
sequence phylogeny of 4 populations of the widely distributed cynomolgus macaque (Macaca fascicularis fascicularis). Journal of Heredity 99:254264.

Bonhomme M, Cuartero S, Blancher A, Crouau-Roy B. 2009. Assessing natural introgression in 2 biomedical model species, the rhesus macaque (Macaca mulatta) and the long-tailed macaque (Macaca fascicularis). Journal of Heredity 100:158-169.

Brandon-Jones D. 1996. The Asian Colobinae as indicators of Quaternary climatic change. Biological Journal of the Linnean Society 59:327-350.

Brunet M, Guy F, Pilbeam D, et al. 2005. New material of the earliest hominid from the Upper Miocene of Chad. Nature 434:752-755.

Cannon CH, Morley RJ, Bush ABG. 2009. The current refugial rainforests of Sundaland are unrepresentative of their biogeographic past and highly vulnerable to disturbance. Proceedings of the National Academy of Sciences of the United States of America 106:11188-11193.

Carbone L, Harris RA, Gnerre S, et al. in press. The gibbon genome gives insights on the fast karyotype evolution of small apes. Nature.

Castresana J. 2000. Selection of conserved blocks from multiple alignments for their use in phylogenetic analysis. Molecular Biology and Evolution 17:540-552.

Deinard A, Smith DG. 2001. Phylogenetic relationships among the macaques: evidence from the nuclear locus NRAMP1. Journal of Human Evolution 41:45-59.

Delson E. 1975. Evolutionary history of the Cercopithecidae. In: Szalay FS, editor. Approaches to primate paleobiology. Contributions to primatology Volume 5. Basel: Karger. p 167-217.

Delson E. 1980. Fossil macaques, phyletic relationships and a scenario of deployment. In: Lindburg DG, editor. The macaques: Studies in ecology, behavior, and evolution. New York: Van Nostrand Reinhold. p 10-30.

de Bruyn M, Stelbrink B, Morley RJ, et al. in press. Borneo and Indochina are major evolutionary hotspots for Southeast Asian biodiversity. Systematic Biology. 
de Ruiter JR, Geffen E. 1998. Relatedness of matrilines, dispersing males and social groups in long-tailed macaques (Macaca fascicularis). Proceedings of the Royal Society B: Biological Sciences 265:79-87.

Drummond AJ, Rambaut A. 2007. BEAST: Bayesian evolutionary analysis by sampling trees. BMC Evolutionary Biology 7:214.

Drummond AJ, Ho SY, Phillips MJ, Rambaut A. 2006. Relaxed phylogenetics and dating with confidence. PLoS Biology 4:e88.

Edgar RC. 2004. MUSCLE: Multiple sequence alignment with high accuracy and high throughput. Nucleic Acids Research 32:1792-1797.

Esselstyn JA, Widmann P, Heaney LR. 2004. The mammals of Palawan Island, Philippines. Proceedings of the Biological Society of Washington 117:271302.

Finstermeier K, Zinner D, Brameier M, et al. 2013. A mitogenomic phylogeny of living primates. PLoS ONE 8:e69504.

Fittinghoff NA, Lindburg DG. 1980. Riverine refuging in east Bornean Macaca fascicularis. In: Lindburg DG, editor. The Macaques: Studies in ecology, behavior and evolution. New York: Van Nostrand Rheinhold. p 215-245.

Fooden J. 1967. Complementary specialization of male and female reproductive structures in the bear macaque, Macaca arctoides. Nature 214:939-941.

Fooden J. 1976. Provisional classification and key to living species of macaques (Primates: Macaca). Folia Primatologica 25:225-236.

Fooden J. 1980. Classification and distribution of living macaques (Macaca Lacepede, 1799). In: Lindburg DG, editor. The Macaques: Studies in ecology, behavior and evolution. New York: Van Nostrand Reinhold. p 1-9.

Fooden J. 1995. Systematic review of Southeast Asian longtail macaques, Macaca fascicularis (Raffles, [1821]). Fieldiana (Zoology) New Series 81:1-206.

Fooden J. 1997. Tail length variation in Macaca fascicularis and M. mulatta. Primates 38:221-231.

Fooden J. 2006. Comparative review of fascicularis-group species of macaques (Primates: Macaca). Fieldiana (Zoology) New Series 107:1-43.

Gelman A, Rubin D. 1992. Inference from iterative simulation using multiple 
sequences. Statistical Science 7:457-472.

Glover I. 1986. Archaeology in eastern Timor, 1966-67. Terra Australis 11:1241.

Gouy M, Guindon S, Gascuel O. 2010. SeaView version 4: A multiplatform graphical user interface for sequence alignment and phylogenetic tree building. Molecular Biology and Evolution 27:221-224.

Groves CP. 2001. Primate taxonomy. Washington, DC: Smithsonian Institution Press. $350 \mathrm{p}$.

Gumert MD, Malaivijitnond S. 2012. Marine prey processed with stone tools by Burmese long-tailed macaques (Macaca fascicularis aurea) in intertidal habitats. American Journal of Physical Anthropology 149:447-457.

Gunnarsdóttir ED, Li M, Bauchet M, Finstermeier K, Stoneking M. 2011. Highthroughput sequencing of complete human mtDNA genomes from the Philippines. Genome Research 21: 1-11.

Guschanski K, Krause J, Sawyer S, et al. 2013. Next-generation museomics disentangle one of the largest primate radiations. Systematic Biology 62: 539-554.

Hall R. 1998. The plate tectonics of Cenozoic SE Asia and the distribution of land and sea. In: Hall R, Holloway JD, editors. Biogeography and geological evolution of SE Asia. Leiden: Backhuys Publishers. p 99-131.

Hall R. 2002. Cenozoic geological and plate tectonic evolution of SE Asia and the SW Pacific: Computer-based reconstructions, model and animations. Journal of Asian Earth Sciences 20:353-431.

Hamada Y, Urasopon N, Hadi I, Malaivijitnond S. 2005. Body size and proportions and pelage color of free-ranging Macaca mulatta from a zone of hybridization in northeastern Thailand. International Journal of Primatology 27:497-513.

Harcourt AH, Meijaard E. 2013. A preliminary test of a prediction from the rafting hypothesis for the presence of non-flying mammals on islands. Journal of Indonesian Natural History 1:23-36.

Harihara S, Saitou N, Hirai M, Aoto N, Terao K, Cho F, Honjo S, Omoto K. 1988. Differentiation of mitochondrial DNA types in Macaca fascicularis. 
Primates 29:117-127.

Haus T, Akom E, Agwanda B, et al. 2013. Mitochondrial diversity and distribution of African green monkeys (Chlorocebus Gray, 1870). American Journal of Primatology 75:350-360.

Haus T, Ferguson B, Rogers J, et al. in press. Genome typing of nonhuman primate models: Implications for biomedical research. Trends in Genetics.

Heaney LR. 1985. Zoogeographic evidence for middle and late Pleistocene land bridges to the Philippine Islands. In: Bartstra G, Casparie WA, editors. Modern Quaternary research in South-East Asia, 9. Rotterdam: A.A. Balkema. p 127-143.

Heaney LR. 1986. Biogeography of mammals in Southeast Asia: Estimates of rates of colonization, extinction, and speciation. Biological Journal of the Linnean Society 28:127-165.

Heaney LR. 1991. A synopsis of climatic and vegetational change in Southeast Asia. Climatic Change 19:53-61.

Hooijer DA. 1962. Quaternary langurs and macaques from the Malay Archipelago. Zoologische Verhandelingen 55:1-64.

Huelsenbeck JP, Ronquist F, Nielsen R, Bollback JP. 2001. Bayesian inference of phylogeny and its impact on evolutionary biology. Science 294:23102314.

Kanthaswamy S, Satkoski J, George D, et al. 2008. Hybridization and stratification of nuclear genetic variation in Macaca mulatta and $M$. fascicularis. International Journal of Primatology 29:1295-1311.

Kanthaswamy S, Ng J, Satkoski Trask J, et al. 2013. The genetic composition of populations of cynomolgus macaques (Macaca fascicularis) used in biomedical research. Journal of Medical Primatology 42:120-31.

Kawamoto Y, Ischak TM, Supriatna J. 1984. Genetic variations within and between troops of the crab-eating macaque (Macaca fascicularis) on Sumatra, Java, Bali, Lombok and Sumbawa, Indonesia. Primates 25:131159.

Kelley J. 2002. The hominoid radiation in Asia. In: Hartwig WC, editor. The primate fossil record. Cambridge: Cambridge University Press. p 369-384. 
Leakey MG. 1993. Evolution of Theropithecus in the Turkana Basin. In: Jablonski NG, editor. Theropithecus, the rise and fall of a primate genus. Cambridge: Cambridge University Press. p 85-124.

Lebatard AE, Bourlès DL, Duringer $P$, et al. 2008. Cosmogenic nuclide dating of Sahelanthropus tchadensis and Australopithecus bahrelghazali: MioPliocene hominids from Chad. Proceedings of the National Academy of Sciences of the United States of America 105:3226-3231.

Li J, Han K, Xing J, et al. 2009. Phylogeny of macaques (Cercopithecidae: Macaca) based on Alu elements. Gene 448:242-249.

Liedigk R, Yang M, Jablonski NG, et al. 2012. Evolutionary history of the oddnosed monkeys and the phylogenetic position of the newly described Myanmar snub-nosed monkey Rhinopithecus strykeri. PLoS ONE 7:e37418.

Liedigk R, Roos C, Brameier M, Zinner D. in press. Mitogenomics of the Old World monkey tribe Papionini. BMC Evolutionary Biology.

Louys J. 2012. Mammal community structure of Sundanese fossil assemblages from the Late Pleistocene, and a discussion on the ecological effects of the Toba eruption. Quaternary International 258:80-87.

Louys J, Meijaard E. 2010. Palaeoecology of Southeast Asian megafaunabearing sites from the Pleistocene and a review of environmental changes in the region. Journal of Biogeography 37:1432-1449.

Maricic T, Whitten M, Pääbo S. 2010. Multiplexed DNA sequence capture of mitochondrial genomes using PCR products. PLoS ONE 5:e14004.

Meijard E. 2003. Mammals of south-east Asia islands and their Late Pleistocene environments. Journal of Biogeography 30: 1245-1257.

Meijaard E, Groves CP. 2004. The biogeographical evolution and phylogeny of the genus Presbytis. Primate Report 68:71-90.

Morales JC, Melnick DJ. 1998. Phylogenetic relationships of the macaques (Cercopithecidae: Macaca), as revealed by high resolution restriction site mapping of mitochondrial ribosomal genes. Journal of Human Evolution 34:1-23. 
Nater A, Nietlisbach P, Arora N, et al. 2011. Sex-biased dispersal and volcanic activities shaped phylogeographic patterns of extant orangutans (genus: Pongo). Molecular Biology and Evolution 28:2275-2288.

Nylander JA, Wilgenbusch JC, Warren DL, Swofford DL. 2008. AWTY (are we there yet?): A system for graphical exploration of MCMC convergence in Bayesian phylogenetics. Bioinformatics 24:581-583.

Perelman P, Johnson WE, Roos C, et al. 2011. A molecular phylogeny of living primates. PLoS Genetics 7:e1001342.

Posada D. 2009. Selection of models of DNA evolution with jModelTest. Methods in Molecular Biology 537:93-112.

Pozzi L, Hodgson JA, Burrell AS, Disotell TR 2011. The stem catarrhine Saadanius does not inform the timing of the origin of crown catarrhines. Journal of Human Evolution 61:209-210.

Pozzi L, Hodgson JA, Burrell AS, et al. 2014. Primate phylogenetic relationships and divergence dates inferred from complete mitochondrial genomes. Molecular Phylogenetics and Evolution 75:165-183.

Pusey AE, Packer C. 1987. Dispersal and philopatry. In: Smuts BB, Cheney DL, Seyfarth RM, Wrangham RW, Struhsaker TT, editors. Primate societies. Chicago: University of Chicago Press. p 250-266.

Rambaut A. 2008. FigTree: Tree figure drawing tool, version 1.2.2. FigTree website. Available: http://tree.bio.ed.ac.uk/software/figtree/.

Rambaut A, Drummond AJ. 2007. Tracer: MCMC trace analysis tool, version 1.5. Tracer website. Available: http://tree.bio.ed.ac.uk/sofware/tracer/.

Riley RP. 2010. The endemic seven: Four decades of research on the Sulawesi macaques. Evolutionary Anthropology 19:22-36.

Rohland N, Siedel H, Hofreiter M. 2004. Nondestructive DNA extraction methods for mitochondrial DNA analyses of museum specimens. Biotechniques 36:814-821.

Rohland N, Siedel H, Hofreiter M. 2010. A rapid column-based ancient DNA extraction method for increased sample throughput. Molecular Ecology Resources 10:677-683.

Rohling EJ, Fenton M, Jorissen FJ, et al. 1998. Magnitude of sea level 
lowstands of the past 500,000 years. Nature 394:162-165.

Ronquist F, Huelsenbeck JP. 2003. MrBayes 3: Bayesian phylogenetic inference under mixed models. Bioinformatics 19:1572-1574.

Roos C, Zinner D. in press. Diversity and evolutionary history of macaques which special focus on rhesus and long-tailed macaques. In: Blümel $\mathrm{J}$, Korte S, Schenck E, Weinbauer GF, editors. The nonhuman primate in nonclinical drug development and safety assessment. New York: Elsevier.

Roos C, Zinner D, Kubatko LS, et al. 2011. Nuclear versus mitochondrial DNA: Evidence for hybridization in colobine monkeys. BMC Evolutionary Biology $11: 77$.

Roos C, Boonratana R, Supriantna J, et al. 2014. An updated taxonomy and conservation status review of Asian primates. Asian Primates Journal 4:238.

Rovie-Ryan JJ, Abdullah MT, Sitam FT, et al. 2013. Y-chromosomal gene flow of Macaca fascicularis (Cercopithecidae) between the insular and mainland peninsula of Penang state, Malaysia. Journal of Science and Technology in the Tropics 9:113-126.

Satkoski Trask JA, Garnica WT, Smith DG, et al. 2013a. Single-nucleotide polymorphisms reveal patterns of allele sharing across the species boundary between rhesus (Macaca mulatta) and cynomolgus ( $M$. fascicularis) macaques. American Journal of Primatology 75:135-144.

Satkoski Trask J, George D, Houghton P, Kanthaswamy S, Smith DG. 2013b. Population and landscape genetics of an introduced species ( $M$. fascicularis) on the island of Mauritius. PLoS ONE 8:e53001.

Shiina T, Tanaka K, Katsuyama Y, et al. 2010. Mitochondrial DNA diversity among three subpopulations of cynomolgus macaques (Macaca fascicularis) originating from the Indochinese Region. Experimental Animals 59:567-578.

Slik JWF, Aiba SI, Ashton PS, et al. 2011. Soils on exposed Sunda Shelf shaped biogeographic patterns in the equatorial forests of Southeast Asia Proceedings of the National Academy of Sciences of the United States of America 108:12343-12347. 
Smith DG, McDonough JW, George DA. 2007. Mitochondrial DNA variation within and among regional populations of long-tail macaques (Macaca fascicularis) in relation to other species of the fascicularis group of macaques. American Journal of Primatology 69:182-198.

Smith DG, Ng J, George D, et al. 2014. A genetic comparison of two alleged subspecies of Philippine cynomolgus macaques. American Journal of Physical Anthropology 155:136-148.

Son D. 2003. Diet of Macaca fascicularis in a mangrove forest, Vietnam. Laboratory Primate News 42:1-5.

Springer MS, Meredith RW, Gatesy J, et al. 2012. Macroevolutionary dynamics and historical biogeography of primate diversification inferred from a species supermatrix. PLoS ONE 7:e49521.

Stamatakis A. 2006. RAxML-VI-HPC: Maximum likelihood-based phylogenetic analyses with thousands of taxa and mixed models. Bioinformatics 22:2688-2690.

Stevison LS, Kohn MH. 2009. Divergence population genetic analysis of hybridization between rhesus and cynomolgus macaques. Molecular Ecology 18:2457-2475.

Stewart AME, Gordon CH, Wich SA, Schroor P, Meijaard E. 2008. Fishing in Macaca fascicularis: A rarely observed innovative behavior. International Journal of Primatology 29:543-548.

Tosi AJ, Coke CS. 2007. Comparative phylogenetics offer new insights into the biogeographic history of Macaca fascicularis and the origin of the Mauritanian macaques. Molecular Phylogenetics and Evolution 42:498504.

Tosi AJ, Morales JC, Melnick DJ. 2000. Comparison of $Y$ chromosome and mtDNA phylogenies leads to unique inferences of macaque evolutionary history. Molecular Phylogenetics and Evolution 17:133-144.

Tosi AJ, Morales JC, Melnick DJ. 2002. Y-chromosome and mitochondrial markers in Macaca fascicularis indicate introgression with Indochinese $M$. mulatta and a biogeographic barrier in the Isthmus of Kra. International Journal of Primatology 23:161-178. 
Tosi AJ, Morales JC, Melnick DJ. 2003. Paternal, maternal, and biparental molecular markers provide unique windows onto the evolutionary history of macaque monkeys. Evolution 57:1419-1435.

van Bemmelen RW. 1970. The geology of Indonesia. General geology of Indonesia and adjacent archipelagoes. The Hague: Martinus Nijhoff. 732 p.

van den Bergh GD, de Vos J, Sondaar PY. 2001. The Late Quaternary palaeogeography of mammal evolution in the Indonesian archipelago. Palaeogeography, Palaeoclimatology, Palaeoecology 171:385-408.

van den Bergh GD, Meijer HJM, Awe RD, et al. 2009. The Liang Bua faunal remains: A 95 k.yr. sequence from Flores, East Indonesia. Journal of Human Evolution 57:527-537.

Vignaud P, Duringer P, Mackaye HT, et al. 2002. Geology and palaeontology of the Upper Miocene Toros-Menalla hominid locality, Chad. Nature 418:152155.

Voris HK. 2000. Maps of Pleistocene sea levels in Southeast Asia: Shorelines, river systems and time durations. Journal of Biogeography 27:1153-1167.

Wheatley BP. 1980. Feeding and ranging of East Bornean Macaca fascicularis. In: Lindburg DG, editor. The Macaques: Studies in ecology, behavior and evolution. New York: Van Nostrand Rheinhold. p 215-245.

Wilting A, Sollmann R, Meijaard E, Helgen KM, Fickel J. 2012. Mentawai's endemic, relictual fauna: Is it evidence for Pleistocene extinctions on Sumatra? Journal of Biogeography 39:1608-1620.

Woodruff DS. 2010. Biogeography and conservation in Southeast Asia: How 2.7 million years of repeated environmental fluctuations affect today's patterns and the future of the remaining refugial-phase biodiversity. Biodiversity and Conservation 19:919-941.

Wyman SK, Jansen RK, Boore JL. 2004. Automatic annotation of organellar genomes with DOGMA. Bioinformatics 20:3252-3255.

Yan G, Zhang G, Fang X, et al. 2011. Genome sequencing and comparison of two nonhuman primate animal models, the cynomolgus and Chinese rhesus macaque. Nature Biotechnology 29:1019-1023. 
Yeager C. 1996. Feeding ecology of the long-tailed macaque (Macaca fascicularis) in Kalimantan Tengah, Indonesia. International Journal of Primatology 17:51-62.

Zalmout IS, Sanders WJ, MacLatchy LM, et al. 2010. New Oligocene primate from Saudi Arabia and the divergence of apes and Old World monkeys. Nature 466:360-365.

Ziegler T, Abegg C, Meijaard E, et al. 2007. Molecular phylogeny and evolutionary history of Southeast Asian macaques forming the $M$. silenus group. Molecular Phylogenetics and Evolution 42:807-816.

Zinner D, Fickenscher GH, Roos C. 2013a. Family Cercopithecidae (Old World Monkeys). In: Mittermeier RA, Rylands AB, Wilson DE, editors. Handbook of the mammals of the world. Volume 3, Primates. Barcelona: Lynx Edicions. p 550-627.

Zinner D, Wertheimer J, Liedigk R, et al. 2013b. Baboon phylogeny as inferred from complete mitochondrial genomes. American Journal of Physical Anthropology 150:133-140. 


\section{Chapter 5}

\section{General Discussion}

\subsection{Summary and discussion}

The cercopithecine tribe Papionini represents one of the most successful primate radiations in terms of diversity and distribution. However, phylogenetic relationships within this tribe are still not satisfactorily resolved and important questions about the evolutionary history of the tribe remain unclear. The objective of my thesis was therefore to contribute to our understanding of the evolution of the Papionini by focussing on the mitogenomic relationships within the tribe. The tribe comprises 45 species of which 20 are represented in this study. Altogether, I generated complete mitochondrial genomes (Sanger sequencing) of 28 Papionini individuals. To generate mitochondrial sequence data, I used blood samples obtained from zoos and breeding facilities (chapter 2) and faecal samples which were collected for earlier studies (Keller et al., 2010; Zinner et al., 2009) (chapter 3). In addition, I gathered dried tissue samples from museum specimens (chapter 4) of which 30 were used to generate mitochondrial genome sequences via DNA-capture and high-throughput sequencing.

To investigate phylogenetic relationships on different taxonomic levels the following three studies were conducted:

(1) An investigation of the mitogenomic relationships and divergence times of all genera (except Rungwecebus) and Macaca species groups of the tribe Papionini (chapter 2). (2) An analysis of the mitogenomic relationships among the six baboon species representing ten different mitochondrial haplogroups to test whether complete mtDNA genomes reveal a better resolution of phylogenetic relationships than previous analyses using only short mtDNA sequences (chapter 3 ). And (3) an analysis of the intra-specific relationships and divergence times among Macaca fascicularis individuals using samples from throughout the species' range (chapter 4).

Results show basically well-resolved and highly supported phylogenies and divergences ages with narrowed confidence intervals. However, the results are in 
some cases contradictory to respective nuclear studies and some relationships still remain ambiguous and need further investigation.

As the main findings of study 1 (chapter 2) we found three mitochondrial clades: (1) Papio, Theropithecus, Lophocebus; (2) Mandrillus, Cercocebus; (3) Macaca. Remarkably, the Macaca clade appears as sister clade to Mandrillus and Cercocebus, a finding that is discordant to morphological and recent nDNA studies (Gilbert, 2008; Perelman et al., 2011; Page \& Goodman, 2001; Xing et al., 2005). A similar finding had been reported from other mtDNA studies (Finstermeier et al., 2013; Pozzi et al., 2014). In this context, we found that an increased taxon sampling can influence phylogenetic results, since alternative positions of the Mandrillus + Cercocebus clade were statistically rejected, which was not the case in Finstermeier et al. (2013).

Our data reveal paraphyletic relationships within the Mandrillus + Cercocebus clade and among Papio taxa, whereas relationships between Theropithecus, Lophocebus and Papio remain ambiguous. The most likely reason for the latter is that the three lineages diverged within a relatively short time period (between 5.2 and 4.7 Ma), which makes a clear resolution difficult. Contemporary gene flow between Papio and Theropithecus was reported (Dunbar \& Dunbar, 1974) and might have also occurred historically and contributed to ambiguous relationships among the three lineages.

The divergence age estimation further revealed initial splits within the three major mitochondrial clades (Papio + Theropithecus + Lophocebus; Mandrillus + Cercocebus; Macaca) at the Miocene/Pliocene boundary and Papionina genera diverged at a similar time scale as Macaca species groups. Hence, the mitochondrial heterogeneity among macaque species groups is at least as high as among African genera. Whether this warrants a respective taxonomic reorganisation is further discussed in section 5.4 .

In study 2 (chapter 3) we found seven major mt-haplogroups among ten baboon populations, indicating paraphyletic relationships of several Papio species. Phylogenetic relationships, especially the most basal splits, remain unresolved although support values were relatively high. This corroborates results from earlier studies which were based on partial mtDNA sequences (cytochrome b, Brown region; Zinner et al., 2009; Keller et al., 2010). Also the obtained divergence dates are consistent with previous studies (Zinner et al., 2009), but appear slightly younger, 
whereas credibility intervals have narrowed. Although node support improved, the obtained mitochondrial tree topology reflects the geographic distribution of respective taxa and not monophyletic species clades. We obtained the same paraphylies and similar divergence times within Papio after combining the respective Papio sequences with the Papionini dataset (chapter 2). The paraphylies among Papio species might be the result of repeated ancient gene flow among different Papio lineages, a process that is still ongoing and reported from most contact zones, especially for olive and hamadryas baboons in Ethiopia and olive and yellow baboons in Amboseli (Alberts \& Altmann, 2001; Bergmann et al., 2008; Nagel, 1973; Shotake, 1981; Tung et al. 2008; Zinner et al., 2011).

In study 3 (chapter 4), we found a continental and an insular Macaca fascicularis clade which separated at $1.7 \mathrm{Ma}$. This finding confirmed results of earlier genetic studies (e.g., Harihara et al., 1988; Tosi et al., 2002, 2003). Furthermore, our mitochondrial data indicate the presence of the continental lineage in North Sumatra. However, whether both haplogroups have restricted ranges or whether both are distributed all over the island remains unknown so far. Tosi and Coke (2007) studied only long-tailed macaques from South Sumatra and found in them only the insular haplogroup, whereas these animals carried both, the continental and the insular Ychromosomal haplogroups. Our study further reveals a rapid radiation of $M$. fascicularis on the Sunda Shelf 0.93-0.84 Ma.

\subsection{The mitochondrial genome and phylogenetic reconstructions}

In summary one can say that complete mitochondrial genomes in general result in mainly well-supported phylogenies, due to the increased number of informative sites compared to shorter mtDNA fragments. However, our results in study 1 and 2 also show some limitations of such approaches, e.g., paraphyletic relationships among baboons and mangabeys and unresolved relationships among Theropithecus, Lophocebus and Papio or the lineages/clades within the M. fascicularis insular clade. Incomplete lineage sorting and secondary gene flow are possible explanations for paraphyletic relationships among baboon and mangabey taxa. Hybridisation has been discussed as reason for tree topology discordances in many studies (e.g., Finstermeier et al., 2013; Keller et al., 2010; Liedigk et al., 2012; Roos et al., 2011; 
Tosi et al., 2002; Zinner et al., 2009, 2011). An example that illustrates discordances between gene trees of Asian colobines as inferred from mitochondrial and nuclear data is shown in Figure 5.1 (Liedigk et al., 2012). The mtDNA phylogeny suggests paraphyly of Rhinopithecus bieti, which is monophyletic in the nDNA phylogeny. A similar pattern is shown among Pygathrix taxa. Both discordances are most likely the result of secondary gene flow between respective lineages. This is just one example showing that the mitochondrial genome represents just one locus and respective phylogenies reveal only a certain, but important aspect of the evolutionary history of taxa. Often the phylogeographic histories of populations or taxa are better preserved in the geographic pattern of mtDNA than in the nuclear genomes of the respective populations (Avise 2000), at least when respective taxa live in female philopatric societies as most primates do (Pusey \& Packer, 1987). Nevertheless, for a complete understanding of the evolutionary history of a species other loci (nDNA) have to be included as well. If such multi-locus nDNA phylogenies are congruent witch each other and with those inferred from mtDNA it could be an indication that the depicted gene trees actually reflect the true species phylogeny (Avise, 2004; Moore, 1995). On the other hand, if nDNA and mtDNA topologies differ, incomplete lineage sorting or secondary gene flow might have influenced the inferred topologies. However, often it remains open, which of both scenarios is responsible for respective discordances. The analyses of whole genomes might contribute to solve this issue and to figure out to which extent hybridisation influenced the evolutionary history of species. With the advent of high-throughput sequencing techniques more and more studies focus on whole genome comparisons, e.g., Gibbs et al., 2007; Higashino et al., 2012; Yan et al., 2011 (macaques), Carbone et al. in press (gibbons), Ebersberger et al., 2002; Li \& Durbin, 2011; Sequencing TC \& AC, 2005 (chimpanzees, humans). In the case of macaques Yan et al. (2011) found out that around $30 \%$ of the Asian mainland $M$. fascicularis genome is of $M$. mulatta origin. Other molecular studies have shown that hybridisation between the M. fascicularis and $M$. sinica lineage formed $M$. arctoides which has a mosaic-like genome comprising genes from both parental lineages (Li et al., 2009; Tosi et al., 2000, 2003; Zinner et al., 2011). Even the human genome has experienced introgression from at least three archaic hominin species (Neanderthals, Denisovans and unknown hominin) (Green et al., 2010; Reich et al., 2010; Prüfer et al. 2014). Single locus analyses often brought up the assumption that introgressive hybridization influenced 
respective phylogenies and with whole genome analyses it is possible to determine more precisely to which extent introgression occurs, since not all parts of the genome are effected equally by hybridisation (Zinner et al., 2011). In this context genomewide Single Nucleotide Polymorphisms (SNPs) detection is of importance, a method to assess genetic variation among populations. Since not all parts of the genome are effected equally by introgression and also not all individuals of a population carry the hybrid genetic make-up in their genome, multi-locus approaches or even the analyses of whole genome data is necessary to resolve, or at least to approximate the complete evolutionary history, hence the species tree of a certain taxon. In the case of gibbons it has recently been shown that even complete genome analysis does not necessarily lead to resolved phylogenies (Carbone et al. in press).

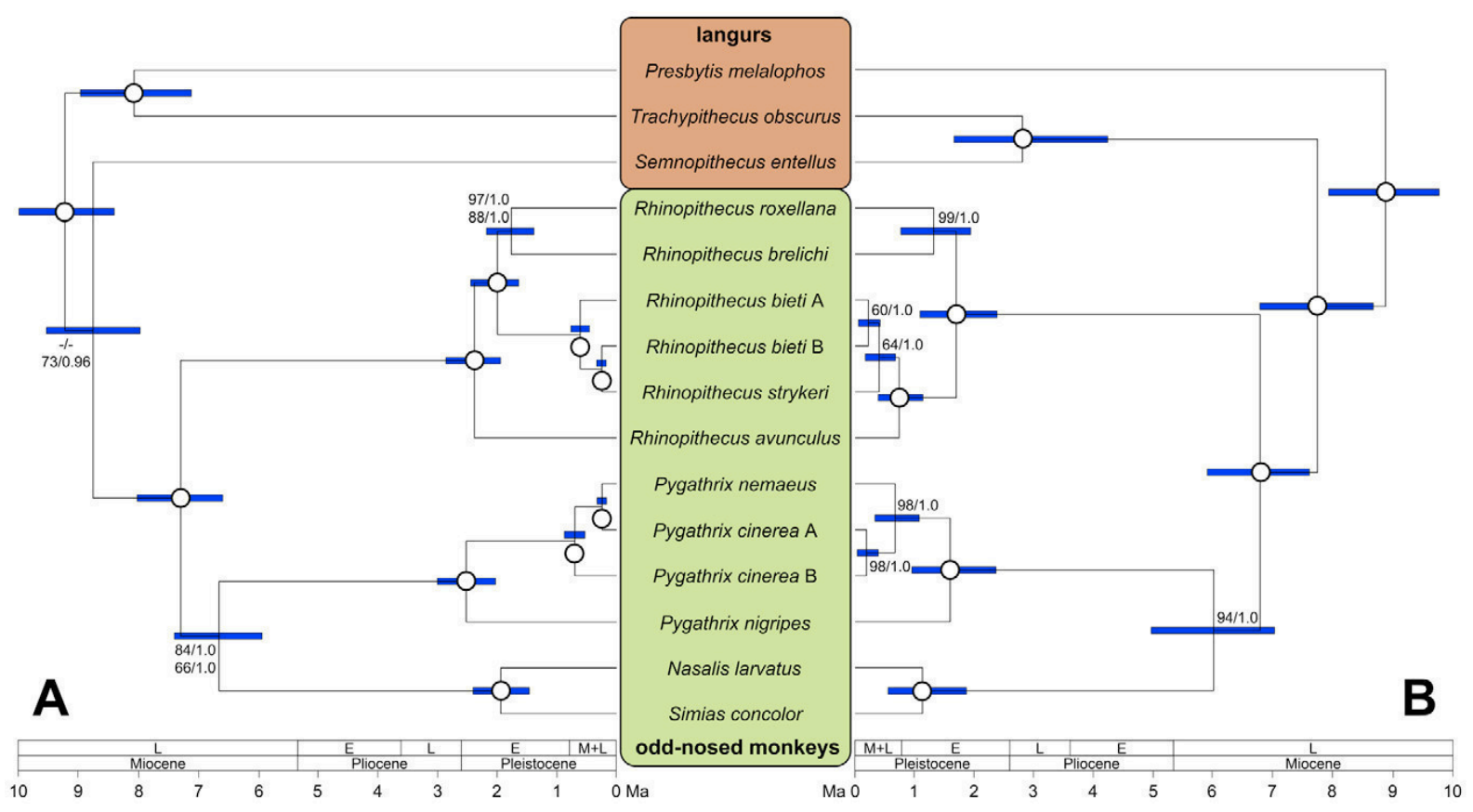

Figure 5.1: Ultrametric tree showing phylogenetic relationships among Asian colobines as obtained from mitochondrial (A) and nuclear sequence data (B). Open circles indicate ML bootstrap values of $100 \%$ and posterior probabilities of 1.0; values below are given at respective branches. Blue bars represent $95 \%$ highest posterior densities of divergence ages. In A, upper and lower numbers on branches indicate ML bootstrap values and posterior probabilities as derived from datasets mtDNA1 and mtDNA2, respectively. Abbreviations used in the bars: $L=$ late, $E=$ early, and $M=$ middle. (Liedigk et al., 2012).

\subsection{Fossils and divergence ages}

Our estimated mitochondrial divergence ages between Papionini genera (chapter 2), between Papio spp. (chapter 3) and between Macaca fascicularis ssp. (chapter 4) 
are mainly in agreement with previous studies (e.g., Finstermeier et al., 2013; Keller et al., 2010; Tosi et al., 2003; Zinner et al., 2009) and further correspond to the papionin fossil record. The earliest macaque fossils outside of Africa, dated to the Late Miocene, were found in southern Europe and were assigned to the M. sylvanus lineage (Alba et al., 2014; Köhler et al., 2000). Our divergence age estimation revealed that the African and the Asian macaque lineages separated around the same time ( $6 \mathrm{Ma})$. This is slightly before guenons (Cercopithecini) left Africa (dated by fossils from Arabia at 6.5-8 Ma; Gilbert et al., 2014). Successive divergence events of the Macaca species groups are mainly in line with the dispersal scenarios proposed by Fooden $(1976,1980)$. The M. silenus lineage (represented in chapter 2 by $M$. silenus and $M$. tonkeana) split off from the remaining macaques at $\sim 5 \mathrm{Ma}$ and formed the first dispersal wave after M. sylvanus. Next, at around $4 \mathrm{Ma}$ the $M$. sinica lineage ( $M$. thibetana) split off, representing Fooden's second dispersal wave. The earliest described macaque fossil in Asia has been dated to $4 \mathrm{Ma}$ (China) (Alba et al., 2014), but was not assigned to any species. The earliest fossils associated with the M. sinica lineage were discovered from Early Pleistocene excavations in China.

According to the estimated divergence ages and the fossil record of Theropithecus, one can infer that the lineage arose in Africa and remained on the continent until the Early Pleistocene (Belmaker, 2002; Delson, 1993; Delson et al., 1993; Delson, 2000; Gibert et al., 1995; Gupta \& Sahni, 1981; Leakey, 1993; Pickford, 1993; Roberts et al., 2014; Rook et al., 2004, 2013).

\subsection{Phylogenetic and taxonomic implications}

One of the main aims of my thesis was to investigate phylogenetic relationships within the Papionini on different taxonomic levels. Some results reveal that defining taxonomic groups is still a challenging task. Due to the mtDNA phylogeny and divergences ages (chapter 2), splits between Macaca species groups are as deep or even deeper as among African genera. Goodman (1998) and Groves (2001; 2004) proposed that taxonomic ranks above species level should be linked to certain time depths. For primates, Groves (2004) suggested for the genus level the Miocene/Pliocene boundary (5.3 Ma), which would correspond to the initial splits in the three Papionini clades and would be in favour of a three-genera-classification. In 
turn, one could argue that macaque species groups should be elevated to genera, given that their African sister taxa are classified on the genus level. However, when considering the morphological similarities of macaques compared to the prominent morphological differences between African genera, a reorganisation of taxonomic ranks of papionins based on time depths seems to be unjustified.

The intra-generic study (chapter 3) revealed paraphyly among almost all baboon species, indicating that complete mitochondrial genomes are not sufficient to identify traditional Papio species as delineated by morphological characters. All six baboon species are clearly distinguishable by morphological characters, but based on mitochondrial markers, they do not form monophyletic groups. Groves (2004) proposed that the criterion of monophyly is mandatory above the species level but not for species. However, mitochondrial paraphyly is also found among genera that are morphologically clearly distinct from each other (e.g., Mandrillus and Cercocebus).

\section{Species concepts}

"A group of actually or potentially interbreeding natural populations which is reproductively isolated from other such groups." This is the well-known definition of the Biological Species Concept (BSC) (Mayr, 1940, 1942, 1963). The BSC is only one species concept among more than 20 others (Groves, 2012), however it is the most prominent one. With regard to results from many former studies the BSC has been proven inapplicable in many cases since interbreeding among different species and even among different genera is a common phenomenon (Zinner et al., 2011).

Alternatively, the Phylogenetic Species Concept (PSC) defines a species as "the smallest cluster of individual organisms within which there is a parental pattern of ancestry and descent and that is diagnosably distinct from other such clusters by a unique combination of fixed character states" (Cracraft, 1983, 1997). In contrast to the BSC, the PSC does not imply the criterion of reproductive isolation, but focuses on any kind of fixed unique characters, be it morphology, behaviour or genetics. According to the PSC, the six baboon species are clearly diagnosable by morphological characters. 


\subsection{Biogeographic implications}

In evolutionary biology, direct observation of evolutionary processes are difficult and in most cases impossible. Therefore, comparative approaches are an important tool for inferring evolutionary history and adaptation (Clutton-Brook \& Harvey, 1984; Harvey \& Purvis, 1991; Harvey \& Pagel, 1991). Furthermore, many questions in evolutionary biology can be studied only if one applies analogues models, e.g. if one uses model species. In order to study the phylogeography of humans, e.g. their dispersal within Africa and out of Africa, two papionin taxa, Papio and Macaca, has been suggested as informative models. The genus Papio, inhabiting most of subSaharan savannah habitats, has evolved in parallel to humans during the last $2.5 \mathrm{Ma}$ in similar, if not in the same habitats as humans (Garrigan \& Kingan, 2007; Jolly, 2001; Kopp et al. in press; Newman et al., 2004) and the genus Macaca can be used as a model for an out-of-Africa dispersal into Eurasia (Andrews et al., 1996; Fooden, 1976, 1980).

Our baboon phylogeny and respective divergence age estimations revealed an initial split into a southern and a northern clade at $~ 2 \mathrm{Ma}$, followed by an east-west split of the northern clade around $1.5 \mathrm{Ma}$. These divisions were most likely triggered by climatic changes and respective transformation of the environment. Similar phylogeographic patterns were also observed in other African savannah mammals, e.g., antelopes (Alcelaphus buselaphus, Connochaetes taurinus, Domaliscus lunatus, Hippotragus equinus), giraffes (Giraffa camelopardalis), warthogs (Phacochoerus africanus), lions (Panthera leo) (Alpers et al., 2004; Arctander et al., 1999; Flagstad et al., 2001; Hassanin et al., 2007; Muwanika et al., 2003; Barnett et al., 2006). Possibly, early savannah-inhabiting humans followed the same dispersal routes during the Pleistocene.

The human lineage dispersed out of Africa most likely during the Early Pleistocene, but the origin and causes of dispersal are not well understood (Antón et al., 2002; Fleagle et al., 2010). Although not on the same time scale, the genus Macaca might represent a useful analogue model to infer human dispersal scenarios (Antón et al., 2002). 


\subsection{Conclusion and outlook}

My thesis provides a comprehensive overview of the mitochondrial diversity among papionin taxa. Although the mitogenomic phylogenies provide normally higher support values than trees derived from shorter mitochondrial sequences, the data further show that the mitochondrial genome as a phylogenetic marker does not allow phylogenetic resolution equally on all taxonomic levels and also does not warrant species delimitation in many cases. Paraphyletic relationships and discordances to nDNA studies indicate that hybridisation and/or incomplete lineage sorting affected most of papionin lineages. To detect hybridisation among the Papionini and to get an approximation to real species trees instead of single gene trees, intensive analyses of nuclear loci or even of whole genomes of the respective taxa are needed. Beside multi-locus approaches, future studies should further increase the sample size per species to reduce phylogenetic error (Nabhan \& Sarkar, 2012; Pollock et al., 2002; Townsend \& Leuenberger, 2011; Zwickl \& Hills, 2002). Emphasis should be placed on primate groups that are understudied in terms of their phylogenetic relationships such as the mangabeys (Cercocebus, Lophocebus, Rungwecebus). Here, in addition to samples from the field, more specimens from museum and other collections should be included in the analyses (e.g. for guenons, Guschanski et al., 2013). These collections provide a comprehensive source of material which can be efficiently analysed with new DNA-capture methods and high-throughput sequencing techniques. Museums might also hold taxa or populations that became already extinct and which cannot be obtained from the field anymore, but which might contribute informative insights to future phylogenetic analyses. Moreover, in addition to molecular data, morphological as well as ecological and behavioural data should be taken into account to get a comprehensive picture of the evolutionary history of taxonomic groups. 


\section{Summary}

The present study is meant to further illuminate the evolutionary history of the cercopithecine tribe Papionini. The Papionini, as sister lineage to the Cercopithecini, belong to the Cercopithecinae subfamily and comprise seven genera (Macaca, Cercocebus, Mandrillus, Lophocebus, Papio, Theropithecus, Rungwecebus) and 45 species. Six of the seven genera are today mainly restricted to Africa with the exception of Papio of which one species, P. hamadryas, is found also in Southwest Arabia. In contrast, the seventh genus, Macaca, is mainly an Asian taxon with only a small range in Northwest Africa ( $M$. sylvanus). The fossil record indicates that the genera Macaca and Theropithecus occurred also in Europe during the PlioPleistocene. Theropithecus is today restricted to Africa but Pliocene fossils were discovered in North India.

Phylogenetic relationships among papionin taxa have been analysed applying morphological and genetic traits, but respective results were not completely concordant. Particularly, the phylogenetic relationships between Theropithecus, Lophocebus and Papio, and relationships within the genus Papio as well as among Macaca species groups are not satisfactorily resolved so far.

To shed more light on the evolutionary history of the Papionini I conducted three studies (chapter 2-4) to investigate inter- and intra-generic as well as intraspecific relationships. For this purpose I generated complete mitochondrial genomes to reconstruct phylogenetic trees and to estimate divergence ages. Results reveal three major clades within the Papionini (chapter 2): (1) Papio, Theropithecus, Lophocebus; (2) Mandrillus, Cercocebus; (3) Macaca, whereas the latter appears as sister clade to Mandrillus and Cercocebus and not as sister lineage to all African Papionini. This finding is in discordance to nuclear and morphological studies. The results further show that complete mitochondrial genomes are in some cases not sufficient to resolve phylogenetic relationships as for example between Theropithecus, Lophocebus and Papio (chapter 2). The dataset further reveals paraphyletic relationships among Mandrillus and Cercocebus (chapter 2) as well as within Papio (chapter 3). In the latter case, baboon mtDNA-clades cluster according to their geographic origin and not according to their taxonomy, making most baboon species paraphyletic. These branching patterns are most likely caused by secondary gene flow between parapatric baboon species. In the third study (chapter 4), in which 
intra-specific relationships within the Asian long-tailed macaque (Macaca fascicularis) were analysed, we found a clear division into a continental and an insular clade. Both, continental and insular lineages were found on Sumatra, indicating secondary gene exchange between continental and insular populations.

In general, the results show that complete mitochondrial genome sequences result in well-resolved and highly supported phylogenies which provide basic phylogenetic information for future comparative studies. Divergence age estimations are mainly concordant with earlier studies but confidence intervals narrowed. However, it has also been shown that mitogenomic phylogenies do not reveal high resolutions when taxa diverge within short time periods. The detected paraphyletic relationships and discordances to nuclear studies are most likely the result of incomplete lineage sorting or secondary gene flow. Hence, for a complete understanding of the evolutionary history of taxa, multi-locus approaches including nuclear data are essential, since mitochondrial phylogenies represent only a single gene tree and thus only one, but important, aspect of the evolutionary history of taxa. 


\section{Zusammenfassung}

Die vorliegende Arbeit soll dazu beitragen, Unstimmigkeiten in den Verwandtschaftsverhältnissen innerhalb der Papionini, einem Stamm innerhalb der Altweltaffen (Cercopithecidae), zu klären. Die Papionini, die zusammen mit den Cercopithecini die Unterfamilie der Cercopithecinae bilden, beinhalten sieben Gattungen (Macaca, Cercocebus, Mandrillus, Lophocebus, Papio, Theropithecus, Rungwecebus) und 45 Arten. Sechs der sieben Gattungen kommen heute hauptsächlich in Afrika vor. Eine Ausnahme ist die Gattung Papio, die mit einer Art ( $P$. hamadryas) auch in Südwest-Arabien vorkommt. Im Gegensatz zu den sechs hauptsächlich afrikanischen Gattungen hat die siebte Gattung (Macaca) nur ein kleines Verbreitungsgebiet im Norden Afrikas und kommt sonst hauptsächlich in Asien vor. Fossilfunde belegen allerdings, dass während des Plio- und Pleistozäns die Gattungen Macaca und Theropithecus auch in Europa vorkamen. Von der Gattung Theropithecus, die heute ausschließlich in Afrika beheimatet ist, wurden zudem auch Fossilien aus dem Pliozän im Norden Indiens gefunden.

Die Verwandtschaftsbeziehungen innerhalb der Papionini wurden bisher mit Hilfe morphologischer und genetischer Merkmale untersucht, allerdings waren die Ergebnisse nicht immer übereinstimmend und es gibt immer noch viele Unklarheiten. Zum einen ist nicht eindeutig geklärt, wie die Gattungen Papio, Lophocebus und Theropithecus zu einander in Beziehung stehen. Zum anderen ist auch unklar, wie die einzelnen Pavianarten innerhalb der Gattung Papio mit einander verwandt sind. Außerdem sind auch die Verwandtschaftsverhältnisse zwischen und innerhalb der Artgruppen der Makaken nicht eindeutig geklärt.

Um mehr Klarheit in die Evolution der Papionini zu bringen, habe ich im Rahmen dieser Arbeit drei Studien durchgeführt (Kapitel 2-4). Ziel dabei war es, Verwandtschaftsbeziehungen auf unterschiedlichen taxonomischen Ebenen (zwischen und innerhalb von Gattungen, sowie innerhalb einer Art) zu untersuchen. Dazu wurden komplette mitochondriale Genome von Vertretern der Papionini sequenziert und damit Phylogenien und Aufspaltungszeiten berechnet. Die Ergebnisse meiner Arbeit zeigen unter anderem drei Hauptkladen innerhalb der Papionini (Kapitel 2): 1) Papio, Theropithecus, Lophocebus; 2) Mandrillus, Cercocebus; 3) Macaca, wobei Macaca in der mitochondrialen Phylogenie näher mit Mandrillus und Cercocebus verwandt zu seien scheint und nicht wie erwartet, als 
Schwestergruppe der afrikanischen Papionini abgebildet wird; ein Ergebnis, das im Widerspruch zu nukleären und morphologischen Studien steht.

Meine Arbeit zeigt auch, dass komplette mitochondriale Genome in manchen Fällen nicht ausreichen, um phylogenetische Beziehungen vollständig zu rekonstruieren. So bleibt weiterhin unklar wie die Gattungen Papio, Theropithecus und Lophocebus zueinander stehen (Kapitel 2). Außerdem zeigen die Ergebnisse Paraphylien für Mandrillus und Cercocebus (Kapitel 2), sowie innerhalb der Paviane (Kapitel 3). Die Paviane werden dabei gemäß inrer geographischen Verbreitung und nicht nach ihrer taxonomischen Zugehörigkeit abgebildet, wodurch die meisten Pavian-Arten paraphyletisch sind. Der Grund für diese Baumtopologie ist sehr wahrscheinlich sekundärer Genfluss zwischen parapatrisch vorkommenden Pavian-Arten. In der dritten Studie (Kapitel 4), in der innerartliche Verwandtschaftsverhältnisse innerhalb einer südostasiatischen Makaken-Artgruppe (Macaca fascicularis) untersucht wurden, zeigt sich eine klare Unterteilung in eine kontinentale und eine insulare Klade. Sowohl die kontinentale, als auch die insulare Linie sind auf Sumatra zu finden, was für einen sekundären genetischen Austausch zwischen beiden Populationen spricht.

Generell kann man sagen, dass komplette mitochondriale Genome robuste Phylogenien mit hoher statistischer Unterstützung ergeben, die eine gute Grundlage für künftige vergleichende Studien bilden. Die berechneten Aufspaltungszeiten stimmen weitestgehend mit vorherigen Studien überein, wobei sich die ermittelten Konfidenzintervalle verkleinert haben. Allerdings zeigt die Arbeit auch, dass Phylogenien basierend auf mitochondrialen Genomen keine hohe Auflösung erzielen wenn sich Taxa innerhalb kurzer Zeit voneinander trennten. Die hier gezeigten Paraphylien und die abweichenden Ergebnisse zu nukleären Studien wurden höchstwahrscheinlich durch sekundären genetischen Austausch hervorgerufen. Um Verwandtschaftsverhältnisse möglichst exakt rekonstruieren zu können, müssen neben der maternal-vererbten, mitochondrialen Linie noch paternal- und biparentalvererbte Merkmale in Betracht gezogen werden. $\mathrm{Zu}$ beachten ist in diesem Zusammenhang, dass ein bestimmter molekularer Marker immer nur eine mögliche Phylogenie von vielen wiedergibt. 


\section{References (Chapter 1 and 5)}

Abegg C, Thierry B. 2002. Macaca evolution and dispersal in insular south-east Asia. Biol J Linn Soc 75:555-576.

Alba DM, Delson E, Carnevale G, Colombero S, Delfino M, Giuntelli P, Pavia M, Pavia G. 2014. First joint record of Mesopithecus and cf. Macaca in the Miocene of Europe. J Hum Evol 67:1-18.

Alberts SC, Altmann J. 2001. Immigration and hybridization patterns of yellow and anubis baboons in and around Amboseli, Kenya. American. J Primatol 53:139-154.

Alpers DL, Van Vuuren BJ, Arctander P, Robinson TJ. 2004. Population genetics of the roan antelope (Hippotragus equinus) with suggestions for conservation. Mol Ecol 13:1771-1784.

Andrews P, Harrison T, Delson E, Bernor RL, Martin L. 1996. Distribution and biochronology of European and Southwest Asian Miocene catarrhines. In (R. L. Bernor, V. Fahlbusch \& H.-W. Mittman, Eds) The Evolution of Western Eurasian Neogene Mammal Faunas, pp. 168-207. New York: Columbia University Press.

Andrews TD, Jermiin LS, Easteal S. 1998. Accelerated Evolution of Cytochrome $b$ in Simian Primates: Adaptive Evolution in Concert with Other Mitochondrial Proteins? J Mol Evol 47:249-257.

Andriaholinirina N, Fausser J-L, Roos C, Ravaoarimanana I, Zinner D, et al. 2006. Molecular phylogeny and taxonomic revision of the sportive lemurs (Lepilemur, Primates). BMC Evol Biol 6, 17. doi:10.1186/1471-2148-6-17.

Antón SC, Leonard WR, Robertson ML. 2002. An ecomorphological model of the initial hominid dispersal from Africa. J Hum Evol 43:773-785.

Arctander P, Johansen C, Coutellec-Vreto MA. 1999. Phylogeography of three closely related African bovids (tribe Alcelaphini). Mol Biol Evol 16:1724-1739.

Avery OT, MacLeod CM, McCarty M. 1944. Studies on the chemical nature of the substance inducing transformation of pneumococcal types induction of transformation by a desoxyribonucleic acid fraction isolated from pneumococcus type III. J Exp Med 79:137-158.

Avise JC. 2000. Phylogeography: the history and formation of species. Cambridge: Harvard University Press.

Avise JC. 2004. Molecular Markers, Natural History, and Evolution. Sunderland, MA: Sinauer Associates.

Barnett R, Yamaguchi N, Barnes I, Cooper A. 2006. The origin, current diversity and future conservation of the modern lion (Panthera leo). Proc Biol Sci, 273:2119-2125.

Beebee TJC, Rowe G. 2008. An Introduction to Molecular Ecology, second edition. Oxford University Press 2:62-64.

Belmaker M. 2002. First evidence of the presence of Theropithecus sp. in the Southern Levant. Israel J Zool 48:165.

Bergman TJ, Phillips-Conroy JE, Jolly CJ. 2008. Behavioral variation and reproductive success of male baboons (Papio anubis x Papio hamadryas) in a hybrid social group. Am J Primatol 70:136-147.

Bonhomme M, Cuartero S, Blancher A, et al. 2009. Assessing natural introgression in 2 biomedical model species, the rhesus macaque (Macaca mulatta) and the long-tailed macaque (Macaca 
fascicularis). J Heredity 100:158-169.

Burrell AS. 2009. Phylogenetics and population genetics of Central African baboons [PhD]. New York: New York University. 323p.

Carbone L, Harris AR, Gnerre S, Veeramah KR, Lorente-Galdos B et al. The gibbon genome gives insights on the fast karyotype evolution of small apes. In press.

Chatterjee HJ, Ho SYW, Barnes I, Groves C. 2009. Estimating the phylogeny and divergence times of primates using a supermatrix approach. BMC Evol Biol 9:259.

Clutton-Brock TH, Harvey PH. 1984. Comparative approaches to investigating adaptation. In: Krebs J, Davies N, editors. Behavioural Ecology. An Evolutionary Approach, 2nd ed. Oxford: Blackwell. p 7-29.

Cracraft J. 1983. Species concepts and speciation analysis. Curr Ornithol 1:159-187.

Cracraft J. 1997. Species concepts in systematics and conservation biology-an ornithological viewpoint. In Claridge, M. F., Dawah, A. A., and Wilson, M. R. (eds.), Species: The Units of Biodiversity, Chapman and Hall, New York, 325-339

Darwin C. 1859. On the Origin of Species by Means of Natural Selection or the Preservation of Favoured Races in the Struggle of Life. John Murray, London.

Degnan JH, Rosenberg NA. 2009. Gene tree discordance, phylogenetic inference and the multispecies coalescent. Trends Ecol Evol 24:332-340.

Delson E. 1974. Preliminary review of cercopithecid distribution in the circum Mediterranean region. Mém Bur Res Geol Min France 78, 131e135.

Delson E. 1975. Evolutionary history of the Cercopithecidae. Contrib Primatol 5:167-217.

Delson E. 1980. Fossil macaques, phyletic relationships and a scenario of deployment. In: The Macaques: Studies in Ecology, Behavior and Evolution Lindhurg D (ed.) New York: Van Nostrand Reinhold, 10-30.

Delson E. 1993. Theropithecus fossils from Africa and India and the taxonomy of the genus. Theropithecus: Rise and Fall of a Primate Genus, Jablonski NG (ed.) Cambridge University Press, Cambridge, 157-189.

Delson E, Eck GG, Leakey MG, Jablonski NG. 1993. A partial catalogue of fossil remains of Theropithecus. In: Theropithecus: The Rise and Fall of a Primate Genus. Jablonski NG (ed.), Cambridge University Press, Cambridge, 499-525.

Delson E. 1996. The oldest monkeys in Asia. International Symposium: Evolution of Asian Primates. Vol. 40.

Delson E. 2000. Cercopithecinae. In: Delson E, Tattersall I, Van Couvering JA, Brooks AS, editors. Encyclopedia of human evolution and prehistory. New York: Garland, 166-171.

Dobzhansky T. 1937. Genetics and the Origin of Species. Columbia University Press, New York.

Dunbar RIM, Dunbar EP. 1974. On hybridization between Theropithecus gelada and Papio anubis in the wild. J Hum Evol 3:187-192.

Ebenau A, Nadler T, Zinner D, Roos C. 2011. Genetic population structure of the critically endangered Delacour's langur in Van Long Nature Reserve, Vietnam. Vietn J Primatol 1:1-15.

Ebersberger I, Metzler D, Schwarz C, Paabo S. 2002. Genomewide comparison of DNA sequences between humans and chimpanzees. Am J Hum Genet 70:1490-1497. 
Erxleben JCP. 1777. Systema regni animalis per classes, ordines, genera, species, varietates cvm synonymia et historia animalivm. Classis I. Mammalia.

Evans BJ, Morales JC, Supriatna J, Melnick DJ. 1999. Origin of the Sulawesi macaques (Cercopithecidae: Macaca) as suggested by mitochondrial DNA phylogeny. Biol J Linn Soc 66:539-560.

Finstermeier K, Zinner D, Brameier M, Meyer M, Kreuz E, Hofreiter M, Roos C. 2013. A mitogenomic phylogeny of living primates. PLoS ONE 578, 8:e69504.

Flagstad O, Syvertsen PO, Stenseth NC, Jakobsen KS. 2001. Environmental change and rates of evolution: The phylogeographic pattern within the hartebeest complex as related to climatic variation. Proc Biol Sci 268:667-677.

Fleagle JG, Shea JJ, Grine FE, Baden AL, Leakey RE. 2010. Out of Africa I. London: Springer.

Fooden J. 1976. Provisional classification and key to living species of macaques (Primates: Macaca). Folia Primatol 25:225-236.

Fooden J. 1980. Classification and distribution of living macaques (Macaca Lacepede, 1799). In: Lindburg DG, editor. The Macaques: Studies in Ecology, Behavior and Evolution. New York: Van Nostrand Reinhold, 1-9.

Frost SR, Marcus LF, Bookstein FL, Reddy DP, Delson E. 2003. Cranial allometry, phylogeography, and systematics of large-bodied papionins (Primates: Cercopithecinae) inferred from geometric morphometric analysis of landmark data. Anatomical Record 275:1048-1072.

Frost SR, Jablonski NG, Haile-Selassie Y. 2014. Early Pliocene Cercopithecidae from Woranso-Mille (Central Afar, Ethiopia) and the origins of the Theropithecus oswaldi lineage. J Human Evol.

Garrigan D, Kingan SB. 2007. Archaic human admixture: A view from the genome. Curr Anthropol, 48:895-902.

Geissmann T. 2003. Vergleichende Primatologie. Springer, Berlin/Heidelberg.

Geissmann T, Lwin N, Aung SS, Aung TN, Aung ZM, Hla TH, Grindley M, Momberg F. 2011. A new species of snubnosed monkey, genus Rhinopithecus Milne-Edwards, 1872 (Primates, Colobinae), from northern Kachin State, northeastern Myanmar. Am J Primatol 73:96-107.

Gervais P. 1859. Zoologie et Paléontologie Françaises, second ed. Bertrand, Paris.

Gibert J, Ribot F, Gibert L, Leakey M, Arribas A, Martinez-Navarro B. 1995. Presence of the cercopithecid genus Theropithecus in Cueva Victoria (Murcia, Spain). J Hum Evol 28:487e493.

Gilbert CC. 2007. Craniomandibular morphology supporting the diphyletic origin of mangabeys and a new genus of the Cercocebus/Mandrillus clade, Procercocebus. J Hum Evol 53:69-102.

Gilbert CC. 2008. African papionin phylogenetic history and Plio-Pleistocene biogeography. PhD thesis. Stony Brook University.

Gilbert CC, Bibi F, Hill A, Beech MJ. 2014. Early guenon from the late Miocene Baynunah Formation, Abu Dhabi, with implications for cercopithecoid biogeography and evolution. Proc Natl Acad Sci U S A 111:10119-10124.

Goodman M, Porter CA, Czelusniak J, Page SL, Schneider H, Shoshani J, Gunnell G, and Groves CP. 1998. Toward a phylogenetic classification of primates based on DNA evidence complemented by fossil evidence. Mol Phylogenet Evol 9:585-598. 
Green RE, Krause J, Briggs AW, Maricic T, Stenzel U, et al. 2010. A draft sequence of the Neandertal genome. Science 328:710-722.

Groves CP. 2001. Primate Taxonomy. Washington, DC: Smithsonian Institution Press.

Groves CP. 2004. The what, why and how of primate taxonomy. Int J Primatol 25:1105- 1126.

Groves C. 2012. Species concepts in primates. Am J Primatol 74:687-691.

Grubb P, Butynski TM, Oates JF, Bearder SK, Disotell TR, Groves C, Struhsaker TT. 2003. Assessment of the diversity of African primates. Int J Primatol 24:1301-1357.

Gupta VJ, Sahni A. 1981. Theropithecus delsoni, a new cercopithecine species from the Upper Siwaliks of India. Bull Ind Geol Ass 14:69-71.

Guschanski K, Krause J, Sawyer S, Valente LM, Bailey S, Finstermeier K, Sabin R, Gilissen E, Sonet G, Nagy ZT, Lenglet G, Mayer F, Savolainen V. 2013. Next generation museomics disentangles one of the largest primate radiations. Syst Biol 62:539-554.

Haeckel E. 1866. Generelle Morphologie der Organismen: Allgemeine Grundzüge der oanischen Formenwissenschaft, mechanisch begründet durch die von Charles Darwin reformirte Descendenztheorie. Band 1: Allgemeine Anatomie der Organismen. Band 2: Allgemeine Entwickelungsgeschichte der Organismen. Berlin: Georg Reimer.

Harihara S, Saitou N, Hirai M, Aoto N, Terao K, Cho F, Honjo S, Omoto K. 1988. Differentiation of mitochondrial DNA types in Macaca fascicularis. Primates 29:117-127.

Hartwig WC. 2002. The primate fossil record (Vol. 33). Cambridge University Press.

Harris EE. 2000. Molecular systematics of the Old World monkey tribe Papionini: analysis of the total available genetic sequences. J Hum Evol 38:235-256.

Harvey PH, Purvis A. 1991. Comparative methods for explaining adaptations. Nature 351:619-624.

Harvey PH, Pagel MD. 1991. The Comparative Method in Evolutionary Biology. Oxford: Oxford University Press.

Hassanin A, Ropiquet A, Gourmand AL, Chardonnet B, Rigoulet J. 2007. Mitochondrial DNA variability in Giraffa camelopardalis : consequences for taxonomy, phylogeography and conservation of giraffes in West and central Africa. C R Biol 330:265-274.

Haus T, Akom E, Agwanda B, Hofreiter M, Roos C, Zinner D. 2013. Mitochondrial diversity and distribution of African green monkeys (Chlorocebus Gray, 1870). Am J Primatol 75:350-360.

Haus T, Ferguson B, Rogers J, Doxiadis G, Certa U, Rose NJ, Teepe R, Weinbauer GF, Roos C. 2014. Genome typing of nonhuman primate models: implications for biomedical research. Trends Genet in press.

Hennig W. 1950. Grundzüge einer Theorie der phylogenetischen Systematik. Deutscher Zentralverlag, Berlin.

Hennig W. 1966. Phylogenetic systematics, University of Chicago, Chicago.

Higashino A, Sakate, R, Kameoka Y, Takahashi I, Hirata M, Tanuma R, Masui T, Yasutomi Y, Osada N. 2012. Whole-genome sequencing and analysis of the Malaysian cynomolgus macaque (Macaca fascicularis) genome. Genome Biol 13:R58.

Jablonski NG, Frost SR. 2010. Cercopithecoidea. In: Werdelin L, Sanders W, editors. Cenozoic mammals of Africa. Berkeley: University of California Press, 393-428.

Jolly CJ. 2001. A proper study for mankind: Analogies from the Papionin monkeys and their 
implications for human evolution. Am J Phys Anthropol 33:177-204.

Jolly CJ, Burrell AS, Phillips-Conroy JE, Bergey C, Rogers J. 2011. Kinda baboons (Papio kindae)

and grayfoot chacma baboons (P. ursinus griseipes) hybridize in the Kafue river valley,

Zambia. Am J Primatol 73:291-303.

Jouffroy FK. 1959. Un crâne subfossile de Macaque du Pleistocène du Viet Nam. Bull Mus Nat Hist Nat, Paris 31:209-216.

Kappeler PM, Rasoloarison RM, Razafimanantsoa L, Walter L, Roos C. 2005. Morphology, behaviour and molecular evolution of giant mouse lemurs (Mirza spp.) Gray, 1870, with description of a new species. Primate Report 71:3-26.

Keller C, Roos C, Groeneveld LF, Fischer J, Zinner D. 2010. Introgressive hybridization in southern African baboons shapes patterns of mtDNA variation. Am J Phys Anthropol 142:125-136.

Kingdon J. 1997. The Kingdon guide to African mammals. London: Academic Press.

Köhler M, Moyà-Solà S, Alba DM. 2000. Macaca (Primates, Cercopithecidae) from the Late Miocene of Spain. J Hum Evol 38:447e452.

Kopp GH, Roos C, Butynski TM, Wildman DE, Alagaili AN, Groenefeld LF, Zinner D. 2014. Out of Africa, but how and when? The case of hamadryas baboons. J Human Evol in press.

Lacépède BGE. 1799. Mémoire sur une nouvelle table méthodique des animaux à mamelles. Mémoires de I'Institut National des Sciences et des Arts. Sciences Mathématiques et Physiques 3: 469-502, 1 table. Paris.

Leakey MG. 1993. Evolution of Theropithecus in the Turkana Basin. In: Jablonski NG, editor. Theropithecus, the rise and fall of a primate genus. Cambridge: Cambridge University Press, 85-124.

Li H, Durbin R. 2011. Inference of human population history from individual whole-genome sequences. Nature, 475(7357), 493-496.

Li J, Han K, Xing J, Kim HS, Rogers J, Ryder OA, Disotell T, Yue B, Batzer MA. 2009. Phylogeny of macaques (Cercopithecidae: Macaca) based on Alu elements. Gene 448:242-249.

Liedigk R, Yang M, Jablonski NG, Momberg F, Geissmann T Lwin N, Htin Hla T, Liu Z, Wong B, Li M, Yongcheng L, Zhang Y-P, Nadler T, Zinner D, Roos C. 2012. Evolutionary history of the oddnosed monkeys and the phylogenetic position of the newly described Myanmar snub-nosed monkey Rhinopithecus strykeri. PLOS ONE 7:e37418. doi:10.1371/ journal.pone.0037418. PubMed: 22616004.

Linnaeus CN. 1758. Systema Naturae Per Regna Tria Naturae, Secundum Classes, Ordines, Genera, Species, Cum Characteribus Differentiis, Synonymis, Locis.

Martin AP, Palumbi SR. 1993. Body size, metabolic rate, generation time, and the molecular clock. Proc Natl Acad Sci USA 90:40874091.

Mayr E. 1940. Speciation phenomena in birds. Am Nat 74:249-278.

Mayr E. 1942. Systematics and the origin of species. New York: Columbia University Press.

Mayr E. 1963. Animal species and evolution. Cambridge: Belknap Press.

Moore WS. 1995. Inferring phylogenies from mtDNA variation: mitochondrial-gene trees versus nuclear-gene trees, Evolution 49:718-726.

Mullis KB, Faloona F, Scharf S, Saiki, R, Horn G, Erlich H. 1986. Specific enzymatic amplification of 
DNA in vitro: the polymerase chain reaction. Cold Spring Harbor Symposia Quant Biol 51:263273.

Muwanika VB, Nyakaana S, Siegismund HR, Arctander P. 2003. Phylogeography and population structure of the common warthog (Phacochoerus africanus) inferred from variation in mitochondrial DNA sequences and microsatellite loci. Heredity 91:361-372.

Nabhan AR, Sarkar IN. 2012. The impact of taxon sampling on phylogenetic inference: a review of two decades of controversy. Brief Bioinform 13:122-134.

Nagel U. 1973. A comparison of anubis baboons, hamadryas baboons and their hybrids at a species border in Ethiopia. Folia Primatol 19:104-165.

Newman TK, Jolly CJ, Rogers J. 2004. Mitochondrial phylogeny and systematics of baboons (Papio). Am J Phys Anthropol 124:17-27.

Page SL, Goodman M. 2001. Catarrhine phylogeny: noncoding DNA evidence for a diphyletic origin of the mangabeys and for a human-chimpanzee clade. Mol Phylogenet Evol 18:14-25.

Pauling L, Corey RB, Branson HR. 1951. The structure of proteins; two hydrogen-bonded helical configurations of the polypeptide chain. Proc Natl Acad Sci USA 37:205-211.

Pickford M. 1993. Climatic change, biogeography, and Theropithecus. In Theropithecus: The Rise and Fall of a Primate Genus. Edited by Jablonski NG. Cambridge: Cambridge University Press, 227-243.

Perelman P, Johnson WE, Roos C, Seuánez HN, Horvath JE, Moreira MAM, Kessing B, Pontius J, Roelke M, Rumpler Y Schneider MPC, Silva A, O'Brien SJ, Pecon-Slattery J. 2011. A molecular phylogeny of living primates. PLoS Genet 7:e1001342.

Pollock DD, Zwickl DJ, Mcguire JA, Hillis DM. 2002. Increased taxon sampling is advantageous for phylogenetic inference. Syst Biol 51:664-671.

Pozzi L, Hodgson JA, Burrell AS, Disotell TR. 2011. The stem catarrhine Saadanius does not inform the timing of the origin of crown catarrhines. Journal of Human Evolution 61:209-210.

Pozzi L, Bergey CM, Burrell AS. 2013. The use (and misuse) of phylogenetic trees in comparative behavioral analyses. Int J Primatol 35:32-54.

Pozzi L, Hodgson JA, Burrell AS, Sterner KN, Raaum RL, Disotell TR. 2014. Primate phylogenetic relationships and divergence dates inferred from complete mitochondrial genomes. Mol Phylogenet Evol 75:165-183.

Prüfer K, Racimo F, Patterson N, Jay F, Sankararaman S, Sawyer S, Heinze A, Renaud G, Sudmant $\mathrm{P}$, de Filippo $\mathrm{C}$ et al. 2014. The complete genome sequence of a Neanderthal from the Altai Mountains. Nature 505:43-49.

Pusey AE, Packer C. 1987. Dispersal and Philopatry. In B. B. Smuts, D. L. Cheney, R. M. Seyfarth, R. W. Wrangham, \& T. T. Struhsaker (Eds.), Primate societies. Chicago: Chicago University Press, 250-266.

Rabarivola C, Zaramody A, Fausser JL, Andriaholinirina N, Roos C, Zinner D, Rumpler Y. 2006. Cytogenetic and molecular characteristics of a new species of Lepilemur from Northern Madagascar. Lemur News 11:45-49.

Reich D, Green RE, Kircher M, Krause J, Patterson N, Durand EY, Viola B, Briggs AW, Stenzel U, Johnson PLF, Maricic T, Good JM, Marques-Bonet T, Alkan C, Fu Q, Mallick S, Li 
H, Meyer M, Eichler EE, Stoneking M, Richards M, Talamo S, Shunkov MV, Derevianko AP, Hublin JJ, Kelso J, Slatkin M, Pääbo S. 2010. Genetic history of an archaic hominin group from Denisova Cave in Siberia. Nature 468:1053-1060.

Riley RP. 2010. The endemic seven: Four decades of research on the Sulawesi macaques. Evol Anthropol 19:22-36.

Roberts P, Delson E, Miracle P, Ditchfield P, Roberts RG, Jacobs Z, Blinkhorn J, Ciochon RL, Fleagle JG, Frost SR, Gilbert CC, Gunnell GF, Harrison T, Korisettar R, Petraglia MD. 2014. Continuity of mammalian fauna over the last $200,000 \mathrm{y}$ in the Indian subcontinent. Proc Natl Acad Sci U S A 111:5848-5853.

Rook L, Martínez-Navarro B, Clark Howell F. 2004. Occurrence of Theropithecus sp. in the Late Villafranchian of Southern Italy and implication for Early Pleistocene "out of Africa" dispersals. J Hum Evol 47:267-277.

Rook L, Martínez-Navarro B. 2013. The large sized cercopithecoid from Pirro Nord and the importance of Theropithecus in the Early Pleistocene of Europe: Faunal marker for hominins dispersal outside Africa. Palaeontographica Abteilung A 298:107-112.

Roos C, Ziegler T, Hodges KJ, Zischler H, Abegg C. 2003. Molecular phylogeny of Mentawai macaques: taxonomic and biogeographic implications. Mol Phylogenet Evol 29:139-150.

Roos C, Nadler T, Walter L. 2008. Mitochondrial phylogeny, taxonomy and biogeography of the silvered langur species group (Trachypithecus cristatus). Mol Phylogenet Evol 47:629-636.

Roos C, Zinner D, Kubatko LS, Schwarz C, Yang M et al. 2011. Nuclear versus mitochondrial DNA: evidence for hybridization in colobine monkeys. BMC Evol Biol 11:77. doi: 10.1186/14712148-11-77. PubMed: 21435245.

Sanger F, Nicklen S, Coulson AR. 1977. DNA sequencing with chain-terminating inhibitors. Proc Natl Acad Sci U S A 74:5463-5467.

Schlosser M. 1924. Fossil primates from China. Palaeont. Sinica, 1:1-16.

Sequencing TC, \& Analysis Consortium. 2005. Initial sequence of the chimpanzee genome and comparison with the human genome. Nature 437:69-87.

Shotake T. 1981. Population genetical study of natural hybridisation between Papio anubis and Papio hamadryas. Primates 22:285-308.

Singer C. 1931. A short history of biology. Oxford.

Sithaldeen R, Bishop JM, Ackermann RR. 2009. Mitochondrial DNA analysis reveals Plio-Pleistocene diversification within the chacma baboon. Mol Phylogenet Evol doi:10.1016/j.ympev. 2009.07.038.

Smith DG, McDonough JW, George DA. 2007. Mitochondrial DNA variation within and among regional populations of long-tail macaques (Macaca fascicularis) in relation to other species of the fascicularis group of macaques. Am J Primatol 69:182-198.

Springer MS, Meredith RW, Gatesy J, Emerling CA, Park J, Rabosky DL, Stadler T, Steiner C, Ryder OA, Janecka JE, Fisher CA, Murphy WJ. 2012. Macroevolutionary dynamics and historical biogeography of primate diversification inferred from a species supermatrix. PLoS ONE 7:e49521.

Stromer E. 1920. Mitteilungen über Wirbeldtierreste aus dem Mittelpliocän des Natrontales (Ägypten). 
5. Nachtrag zu I. Affen. Sitz.-ber. Bayerischen Adak Wissen Mat-phys $345 \mathrm{e} 370$.

Sutton WS. 1903. The chromosomes in heredity. Biol Bull 4:231-251.

Tattersall I. 2007. Madagascar's lemurs: cryptic diversity or taxonomic inflation? Evol Anthropol 16: 12-23.

Thalmann O, Hebler J, Poinar HN, Pääbo S, Vigilant L. 2004. Unreliable mtDNA data due to nuclear insertions: a cautionary tale from analysis of humans and other great apes. Mol Ecol 13:321335.

Thinh VN, Mootnick AR, Thanh VN, Nadler T, Roos C. 2010a. A new species of crested gibbon, from the central Annamite mountain range. Vietn J Primatol 1:1-12.

Thinh VN, Rawson B, Hallam C, Kenyon M, Nadler T, Walter L, Roos C. 2010b. Phylogeny and distribution of crested gibbons (genus Nomascus) based on mitochondrial cytochrome b gene seq uence data. Am J Primatol 72:1047-1054.

Tosi AJ, Morales JC, Melnick DJ. 2000. Comparison of $Y$ chromosome and mtDNA phylogenies leads to unique inferences of macaque evolutionary history. Mol Phylogenet Evol 17:133-144.

Tosi AJ, Morales JC, Melnick DJ. 2002. Y-chromosome and mitochondrial markers in Macaca fascicularis indicate introgression with Indochinese M. mulatta and a biogeographic barrier in the Isthmus of Kra. Int JPrimatol 23:161-178.

Tosi AJ, Morales JC, Melnick DJ. 2003. Paternal, maternal, and biparental molecular markers provide unique windows onto the evolutionary history of macaque monkeys. Evolution 57:1419-1435.

Tosi AJ, Coke CS. 2007. Comparative phylogenetics offer new insights into the biogeographic history of Macaca fascicularis and the origin of the Mauritanian macaques. Mol Phylogenet Evol 42:498-504.

Townsend JP, Leuenberger C. 2011. Taxon sampling and the optimal rates of evolution for phylogenetic inference. Syst Biol 60:358-365.

Tung J, Charpentier MJE, Garfield D, Altmann J, Alberts SC. 2008. Genetic evidence reveals temporal change in hybridization patterns in a wild baboon population. Mol Ecol 17:1998-2011.

Wallace A. 1858. On the Tendency of Varieties to Depart Indefinitely from the Original Type. Proc Linn Soc 3:53-62.

Watson JD, Crick FH. 1953. Molecular structure of nucleic acids. Nature 171:737-738.

Wiesner RJ, Rüegg JC, Morano I. 1992. Counting target molecules by exponential polymerase chain reaction: copy number of mitochondrial DNA in rat tissues. Biochem Biophys Res Commun 183:553-559.

Williams BA, Ross CF, Frost SR, Waddle DM, Gabadirwe M, Brook GA. 2012. Fossil Papio cranium from !Ncumtsa (Koanaka) Hills, western Ngamiland, Botswana. Am J Phys Anthropol 149:117.

Wolstenholme, DR. 1992. Animal mitochondrial DNA: structure and evolution. Int Rev Cytol 141:173216.

Xing J, Wang H, Han K, Ray DA, Huang CH, Chemnick LG, Stewart CB, Disotell TR, Ryder OA, Batzer MA. 2005. A mobile element based phylogeny of Old World monkeys. Mol Phylogenet Evol 37:872-880. 
Yoder AD, Vilgalys R, Ruvolo M. 1996. Molecular evolutionary dynamics of cytochrome $b$ in strepsirrhine primates: the phylogenetic significance of third-position transversions. Mol Biol Evol 13:1339-1350.

Yan G, Zhang G, Fang X, Zhang Y, Li C, Ling F, ... Wang J, et al. 2011. Genome sequencing and comparison of two nonhuman primate animal models, the cynomolgus and Chinese rhesus macaques. Nat Biotechnol 29:1019-1025.

Yang M, Yang Y, Cui D, Fickenscher G, Zinner D, Roos C, Brameier M. 2012. Population genetic structure of Guizhou snub-nosed monkeys (Rhinopithecus brelichi) as inferred from mitochondrial control region sequences, and comparison with $R$. bieti and $R$. roxellana. Am J Phys Anthropol 147: 1-10.

Young C. 1934. On the Insectivora, Chiroptera, Roddentia and Primates other than Sinanthropus from locality 1 at Choukoutien. Palaeont Sinica, ser. C, 8:1-160.

Zalmout IS, Sanders WJ, MacLatchy LM, Gunnell GF, Al-Mufarreh YA, Ali MA, Nasser AAH, Al-Masari AM, Al-Sobhi SA, Nadhra AO, Matari AH, Wilson JA, Gingerich PD. 2010. New Oligocene primate from Saudi Arabia and the divergence of apes and Old World monkeys. Nature 466:360-365.

Zaramody A, Fausser JL, Roos C, Zinner D, Andriaholinirina N, Rabarivola C, Norscia I, Tattersall I, Rumpler Y. 2006. Molecular phylogeny and taxonomic revision of the eastern woolly lemurs (Avahi laniger). Primate Report 74: 9-23.

Zhang Y, Ryder OA. 1998. Mitochondrial Cytochrome b Gene Sequences of Old World Monkeys: With Special Reference on Evolution of Asian Colobines. Primates, 39(1): 39-49.

Ziegler T, Abegg C, Meijaard E, Perwitasari-Farajallah D, Walter L, Hodges, JK, Roos C. 2007. Molecular phylogeny and evolutionary history of Southeast Asian macaques forming the $M$. silenus group. Mol Phylogent Evol 42:807-816.

Zinner D, Groeneveld LF, Keller C, Roos C. 2009. Mitochondrial phylogeography of baboons (Papio spp.)-indication for introgressive hybridization? BMC Evol Biol 9:83.

Zinner D, Arnold ML, Roos C. 2009. Is the new primate genus Rungwecebus a baboon?. PLoS One 4:e4859.

Zinner D, Buba U, Nash S, Roos C. 2011. Pan-African voyagers. The phylo-geography of baboons. In: Sommer V, Ross C, editors. Primates of Gashaka. Socioecology and conservation in Nigeria's biodiversity hotspot. New York: Springer, 267-306.

Zinner D, Arnold ML, Roos C. 2011. The strange blood: natural hybridization in primates. Evol Anthropol 20:96-103. doi:10.1002/evan. 20301. PubMed: 22034167.

Zinner D, Fickenscher GH, Roos C. 2013. Family Cercopithecidae (Old World Monkeys). In: Mittermeier RA, Rylands AB, Wilson DE, editors. Handbook of the Mammals of the World. Vol.

3. Primates. Barcelona: Lynx Edicions, 550-627.

Zukerkandl E, Pauling L. 1965. Evolutionary divergence and convergence in proteins. In Evolving Genes and Proteins (V Bryson \& HJ Vogel, eds), pp. 97166. Academic Press, New York.

ZwickI DJ, Hillis DM. 2002. Increased taxon sampling greatly reduces phylogenetic error. Syst Biol 51:588-598.

Zwickl DJ. 2006. Genetic algorithm approaches for the phylogenetic analysis of large biological 
sequence data sets under themaximum likelihood criterion. PhD Thesis. Austin: University of Texas. 


\section{Chapter 2}

Table S1. Divergence ages among catarrhine primates in Ma (95\% credibility intervals) estimated with uncorrelated and auto-correlated relaxed clock models based on dataset 1.

\begin{tabular}{|c|c|c|c|c|}
\hline & uncorrelated (BEAST) & $\begin{array}{l}\text { uncorrelated } \\
\text { (PhyloBayes) }\end{array}$ & $\begin{array}{c}\text { autocorrelated } \\
\text { (PhyloBayes) }\end{array}$ & \\
\hline Split & $\begin{array}{c}\text { mtDNA divergence } \\
\text { ages }\end{array}$ & $\begin{array}{c}\text { mtDNA divergence } \\
\text { ages }\end{array}$ & $\begin{array}{c}\text { mtDNA divergence } \\
\text { ages }\end{array}$ & $\begin{array}{l}\text { nDNA divergence } \\
\text { ages }^{1}\end{array}$ \\
\hline Cercopithecoidea - Hominoidea & $27.12(23.62-30.83)$ & $27.42(24.23-30.76)$ & $24.62(24.02-26.19)$ & $31.56(25.66-37.88)$ \\
\hline Pongo - (Homo + Pan $)$ & $13.86(12.74-15.01)$ & $13.82(13.04-14.90)$ & $13.75(13.03-14.88)$ & $16.52(13.45-19.68)$ \\
\hline Homo - Pan & $6.41(5.81-6.98)$ & $6.45(6.02-6.97)$ & $6.59(6.04-6.98)$ & $6.60(5.40-7.96)$ \\
\hline Colobinae - Cercopithecinae & $18.47(13.89-24.03)$ & $22.66(18.21-27.29)$ & $15.78(13.00-18.87)$ & $17.57(13.88-21.52)$ \\
\hline $\begin{array}{l}\text { Papionini - Chlorocebus } \\
\text { (Cercopithecini) }\end{array}$ & $12.51(9.77-15.72)$ & $15.58(12.03-19.69)$ & $11.50(9.53-13.68)$ & $11.50(9.18-13.85)$ \\
\hline $\begin{array}{l}\text { (Macaca + Mandrillus + } \\
\text { Cercocebus) }-(\text { Papio }+ \\
\text { Theropithecus + Lophocebus) }\end{array}$ & $10.69(8.24-13.20)$ & $12.68(9.83-16.31)$ & $10.21(8.69-11.95)$ & - \\
\hline Macaca - remaining Papionini & - & - & - & $8.13(6.69-9.68)$ \\
\hline $\begin{array}{l}\text { (Mandrillus + Cercocebus) - } \\
\text { (Papio + Lophocebus + } \\
\text { Theropithecus) }\end{array}$ & - & - & - & $6.67(5.37-8.07)$ \\
\hline $\begin{array}{l}\text { Macaca - (Mandrillus + } \\
\text { Cercocebus) }\end{array}$ & $9.41(7.34-11.91)$ & $10.82(8.29-14.14)$ & $9.45(8.23-10.81)$ & - \\
\hline $\begin{array}{l}\text { Theropithcus - (Papio + } \\
\text { Lophocebus) }\end{array}$ & $5.20(4.04-6.41)$ & $5.24(3.79-6.42)$ & $6.11(5.28-6.49)$ & $4.06(3.36-4.70)$ \\
\hline Papio-Lophocebus & $4.70(3.59-5.92)$ & $4.70(3.33-6.04)$ & $5.87(5.06-6.35)$ & $3.24(2.46-4.07)$ \\
\hline $\begin{array}{l}\text { T. gelada } 2-(T . \text { gelada } 3+T . \\
\text { gelada 1) }\end{array}$ & $0.30(0.16-0.50)$ & $0.30(0.13-0.69)$ & $1.93(0.89-3.13)$ & - \\
\hline T. gelada $3-$ T. gelada 1 & $0.04(0.02-0.08)$ & $0.04(0.01-0.12)$ & $0.80(0.21-1.83)$ & - \\
\hline $\begin{array}{l}\text { C. atys }-(C . \text { torquatus }+C \text {. } \\
\text { chrysogaster }+M . \text { sphinx }+M \text {. } \\
\text { leucophaeus })\end{array}$ & $4.19(3.02-5.43)$ & $4.61(2.85-7.30)$ & $4.87(3.39-6.03)$ & - \\
\hline $\begin{array}{l}\text { C. torquatus }-(M . \text { sphin } x+C . \\
\text { chrysogaster }+M \text {. leucophaeus })\end{array}$ & $3.59(2.58-4.75)$ & $3.82(2.33-6.11)$ & $4.29(2.83-5.51)$ & - \\
\hline $\begin{array}{l}\text { M. sphinx - (C. chrysogaster + } \\
\text { M. leucophaeus) }\end{array}$ & $2.67(1.88-3.67)$ & $2.74(1.60-4.56)$ & $3.36(2.05-4.52)$ & - \\
\hline $\begin{array}{l}\text { C. chrysogaster }-M \text {. } \\
\text { leucophaeus }\end{array}$ & $1.85(1.13-2.74)$ & $1.85(0.94-3.29)$ & $2.63(1.49-3.73)$ & - \\
\hline Mandrillus - Cercocebus & - & - & - & $4.85(3.58-6.23)$ \\
\hline M. sylvanus - Asian macaques & $5.93(4.95-6.93)$ & $5.93(4.84-6.48)$ & $6.29(5.77-6.50)$ & $5.12(4.27-5.93)$ \\
\hline $\begin{array}{l}(M . \text { silenus }+M . \text { tonkeana })- \\
\text { remaining macaques }\end{array}$ & $5.16(4.18-6.15)$ & $5.19(4.13-6.01)$ & $5.89(5.30-6.26)$ & $4.13(3.26-5.01)$ \\
\hline $\begin{array}{l}\text { M. silenus - M. tonkeana } \\
\text { (Sulawesi macaques) }\end{array}$ & $3.34(2.11-4.51)$ & $3.23(1.91-4.58)$ & $4.58(3.79-5.19)$ & $3.13(2.35-3.98)$ \\
\hline $\begin{array}{l}\text { M. thibetana }-(M . \text { mulatta }+M \text {. } \\
\text { arctoides }+M \text {. fascicularis })\end{array}$ & $3.97(3.09-4.90)$ & $3.93(2.90-4.98)$ & $5.04(4.41-5.52)$ & - \\
\hline $\begin{array}{l}\text { M. fascicularis - (M. mulatta }+ \\
\text { M. arctoides) }\end{array}$ & $3.28(2.49-4.14)$ & $3.18(2.22-4.20)$ & $4.61(3.97-5.10)$ & - \\
\hline $\begin{array}{l}\text { M. fascicularis } 2-(M . \\
\text { fascicularis } 1+M \text {. fascicularis } \\
\text { 3) }\end{array}$ & $1.08(0.76-1.51)$ & $1.14(0.62-1.99)$ & $2.28(1.61-3.00)$ & - \\
\hline $\begin{array}{l}\text { M. fascicularis } 1-M \text {. } \\
\text { fascicularis } 3\end{array}$ & $0.60(0.43-0.77)$ & $0.65(0.32-1.26)$ & $1.12(0.72-1.61)$ & - \\
\hline M. mulatta - M. arctoides & $2.86(2.14-3.72)$ & $2.70(1.78-3.68)$ & $4.32(3.67-4.84)$ & - \\
\hline $\begin{array}{l}\text { M. mulatta 2 - (M. mulatta } 1+ \\
\text { M. mulatta 3) }\end{array}$ & $1.56(0.93-2.30)$ & $1.41(0.74-2.35)$ & $2.94(2.27-3.50)$ & - \\
\hline M. mulatta $1-M$. mulatta 3 & $0.02(0.01-0.05)$ & $0.02(0.01-0.06)$ & $0.09(0.02-0.30)$ & - \\
\hline M. thibetana $1-M$. thibetana 2 & $0.04(0.01-0.07)$ & $0.03(0.01-0.08)$ & $0.26(0.04-0.97)$ & - \\
\hline M. sylvanus $1-M$. sylvanus 2 & $0.04(0.02-0.07)$ & $0.04(0.01-011)$ & $0.36(0.04-1.30)$ & - \\
\hline $\begin{array}{l}\text { (M. arctoides }+M . \text { thibetana })- \\
(M . \text { fascicularis }+M . \text { mulatta })\end{array}$ & - & - & - & $3.53(2.69-4.47)$ \\
\hline M. fascicularis - M. mulatta & - & - & - & $2.77(1.94-3.67)$ \\
\hline M. arctoides $-M$. thibetana & - & - & - & $2.38(1.40-3.37)$ \\
\hline
\end{tabular}




\begin{tabular}{|c|c|c|c|c|}
\hline $\begin{array}{l}\text { Papio ursinus south - remaining } \\
\text { baboons }\end{array}$ & $2.22(1.67-2.81)$ & $2.01(1.30-3.11)$ & $3.31(2.51-3.97)$ & - \\
\hline $\begin{array}{l}\text { ( } P \text {. ursinus north }+P \text {. } \\
\text { cynocephalus south }+P \text {. } \\
\text { kindae }) \text { - remaining baboons }\end{array}$ & $1.98(1.51-2.52)$ & $1.84(1.21-2.83)$ & $3.18(2.37-3.84)$ & - \\
\hline $\begin{array}{l}P . \text { kindae }-(P . \text { ursinus north }+ \\
P . \text { cynocephalus south })\end{array}$ & $1.41(0.99-1.86)$ & $1.27(0.73-2.07)$ & $2.52(1.78-3.17)$ & - \\
\hline $\begin{array}{l}P \text {. ursinus north }-P \text {. } \\
\text { cynocephalus south }\end{array}$ & $0.68(0.37-1.08)$ & $0.61(0.23-0.28)$ & $1.36(0.83-1.92)$ & - \\
\hline $\begin{array}{l}\text { ( } P \text {. anubis west }+P \text {. papio }+P \text {. } \\
\text { anubis west } 1)-(P \text {. anubis east } \\
+P \text {. hamadryas }+P \text {. } \\
\text { cynocephalus north })\end{array}$ & $1.44(1.07-1.89)$ & $1.29(0.81-2.05)$ & $2.51(1.77-3.18)$ & - \\
\hline $\begin{array}{l}\text { (P. anubis west } 2+P . \text { papio })- \\
P . \text { anubis west } 1\end{array}$ & $1.18(0.82-1.58)$ & $1.02(0.60-1.67)$ & $2.21(1.52-2.86)$ & - \\
\hline P. anubis west $2-P$. papio & $1.10(0.74-1.49)$ & $0.94(0.52-1.54)$ & $2.13(1.45-2.78)$ & - \\
\hline $\begin{array}{l}\text { (P. anubis east }+P . \text { hamadryas } \\
1+P . \text { hamadryas } 2)-P . \\
\text { cynocephalus north }\end{array}$ & $0.40(0.26-0.58)$ & $0.37(0.20-0.69)$ & $0.89(0.46-1.49)$ & - \\
\hline $\begin{array}{l}P . \text { hamadryas } 2-(P . \text { anubis } \\
\text { east }+P \text {. hamadryas } 1)\end{array}$ & $0.25(0.16-0.37)$ & $0.21(0.11-0.40)$ & $0.58(0.29-1.04)$ & - \\
\hline $\begin{array}{l}\text { P. anubis east }-P \text {. hamadryas } \\
1\end{array}$ & $0.21(0.12-0.33)$ & $0.18(0.09-0.35)$ & $0.52(0.25-0.92)$ & - \\
\hline $\begin{array}{l}P . \text { papio }-(P . \text { anubis }+P . \\
\text { hamadryas })\end{array}$ & - & - & - & $1.21(0.70-1.79)$ \\
\hline P. anubis - P. hamadryas & - & - & - & $0.72(0.35-1.17)$ \\
\hline
\end{tabular}

${ }^{1}$ nuclear divergence ages from [15] based on 34,927 bp from 54 genes.

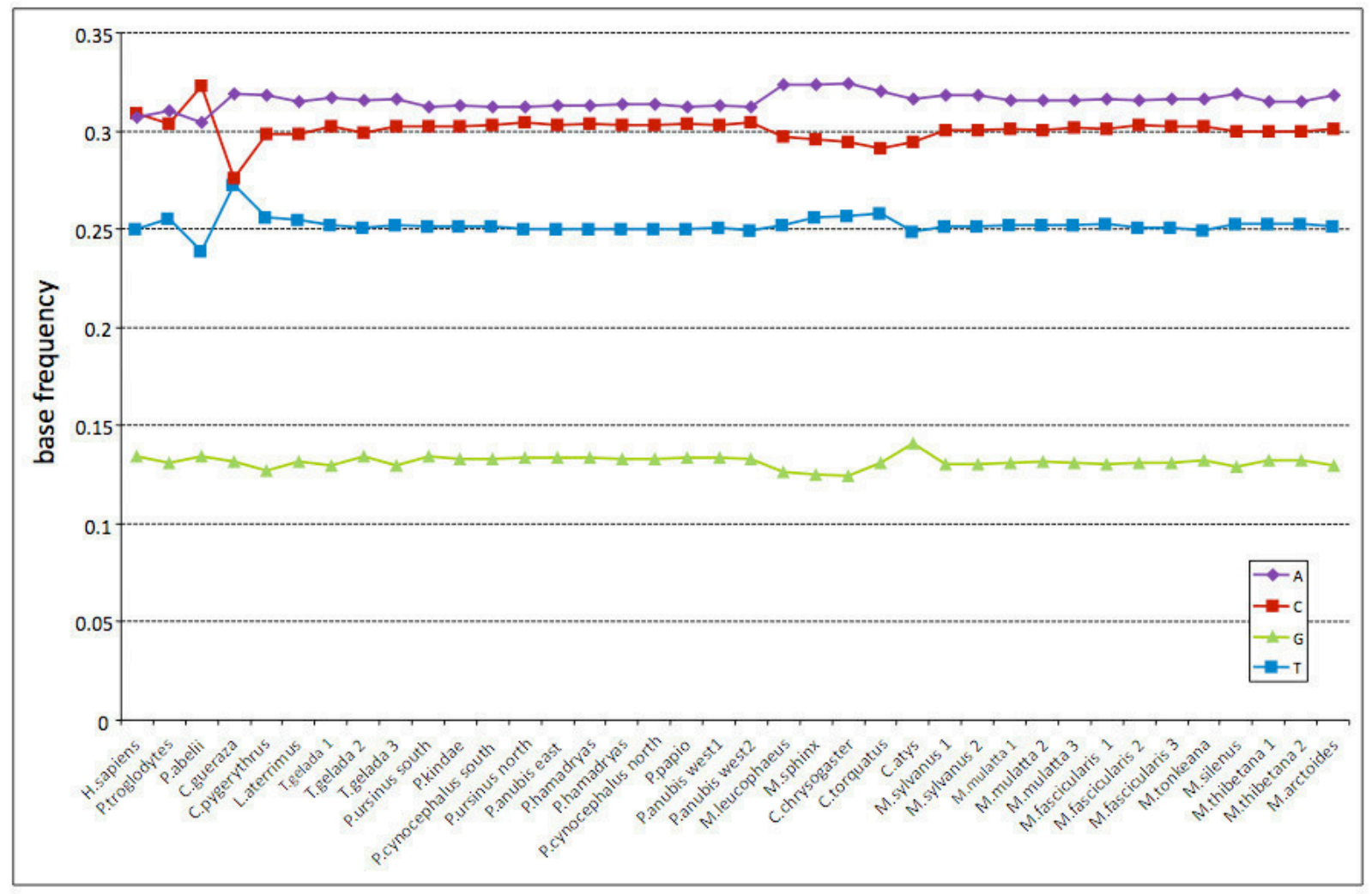

Figure S1. Nucleotide composition among Papionini and outroup taxa. 


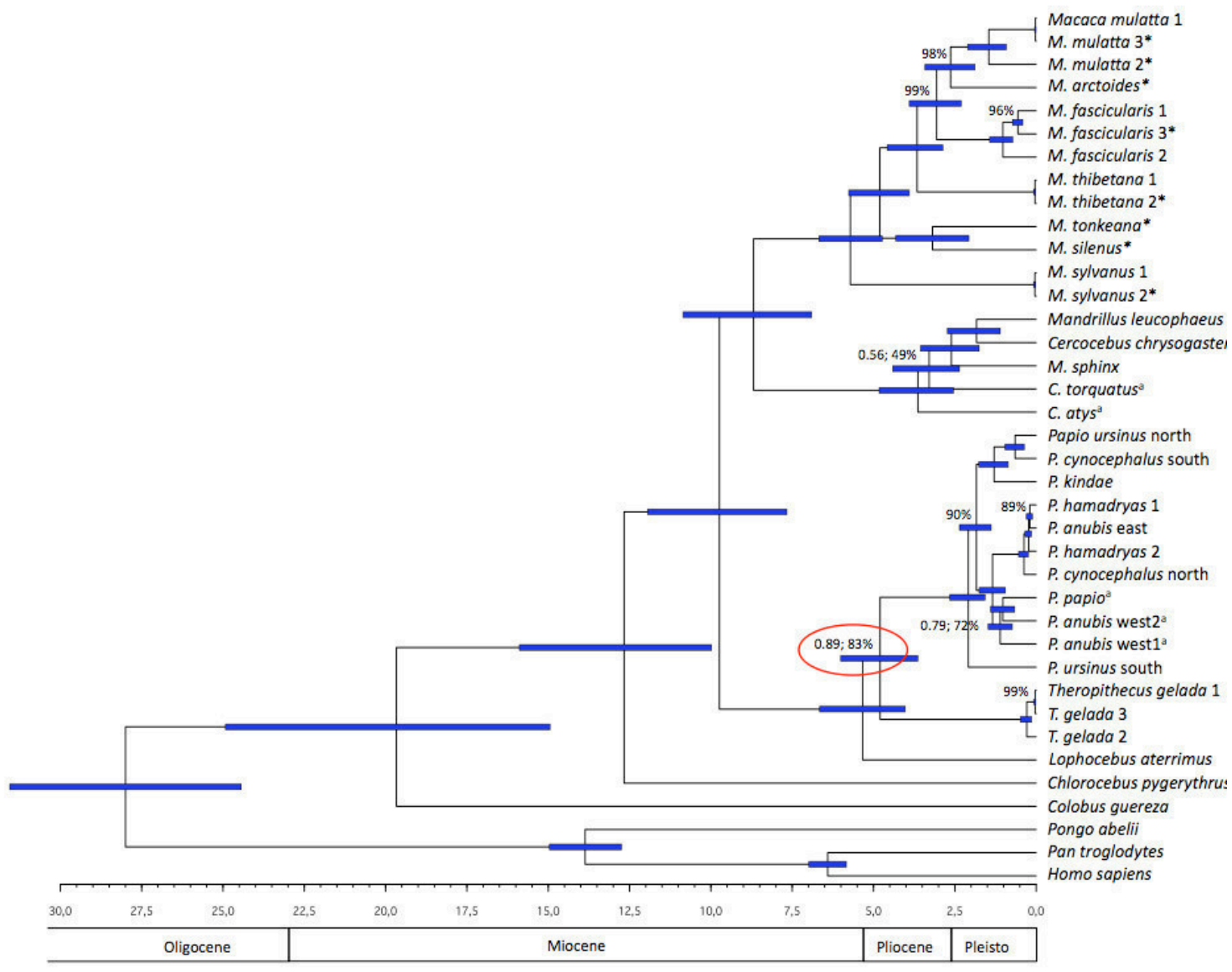

Figure S2. Ultrametric tree of Papionini and outgroup taxa as inferred from dataset 2. Tree topologies as inferred from Bayesian (MrBayes) as well as from ML (RAxML) estimations were mainly identical with some exceptions. All unlabelled branches show ML BP of $100 \%$ and Bayesian PP of 1.0. Values below are indicated at respective nodes. Taxa indicated with a are arranged differently in the ML (RAxML) and Bayesian tree (MrBayes): (( $P$. anubis west2, $P$. anubis west1) P. papio); ((C. torquatus, C. atys), ((C. chrysogaster, M. leucophaeus), M. sphinx)). Red ellipse indicates main difference to Figure $1 .{ }^{*}$ = sequences were newly generated in this study. 


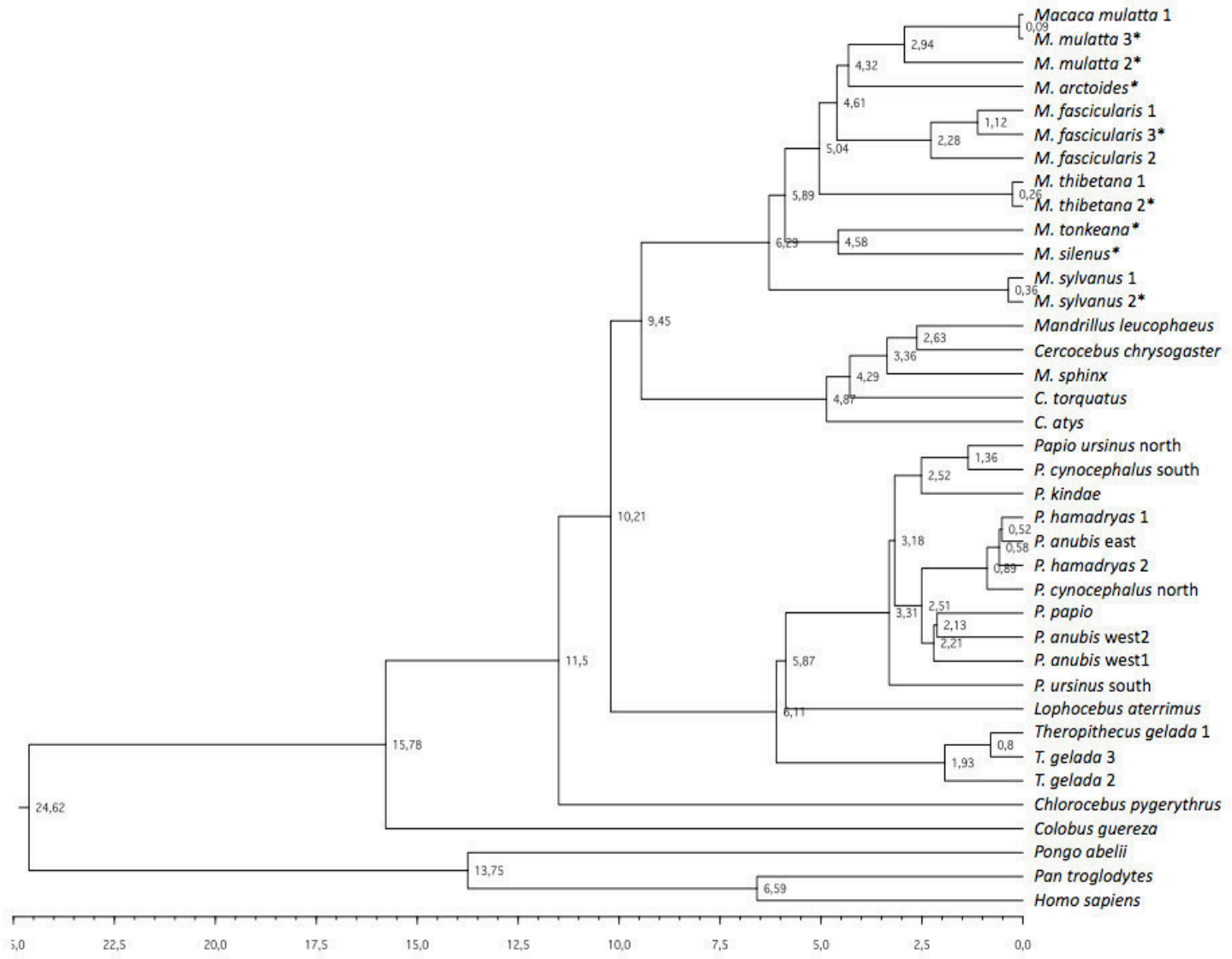

Figure S3. Tree topology including divergence dates as estimated with an auto-correlated relaxed clock model as implemented in PhyloBayes 3.3. Time scale shows million years before present. * sequences were newly generated in this study. 


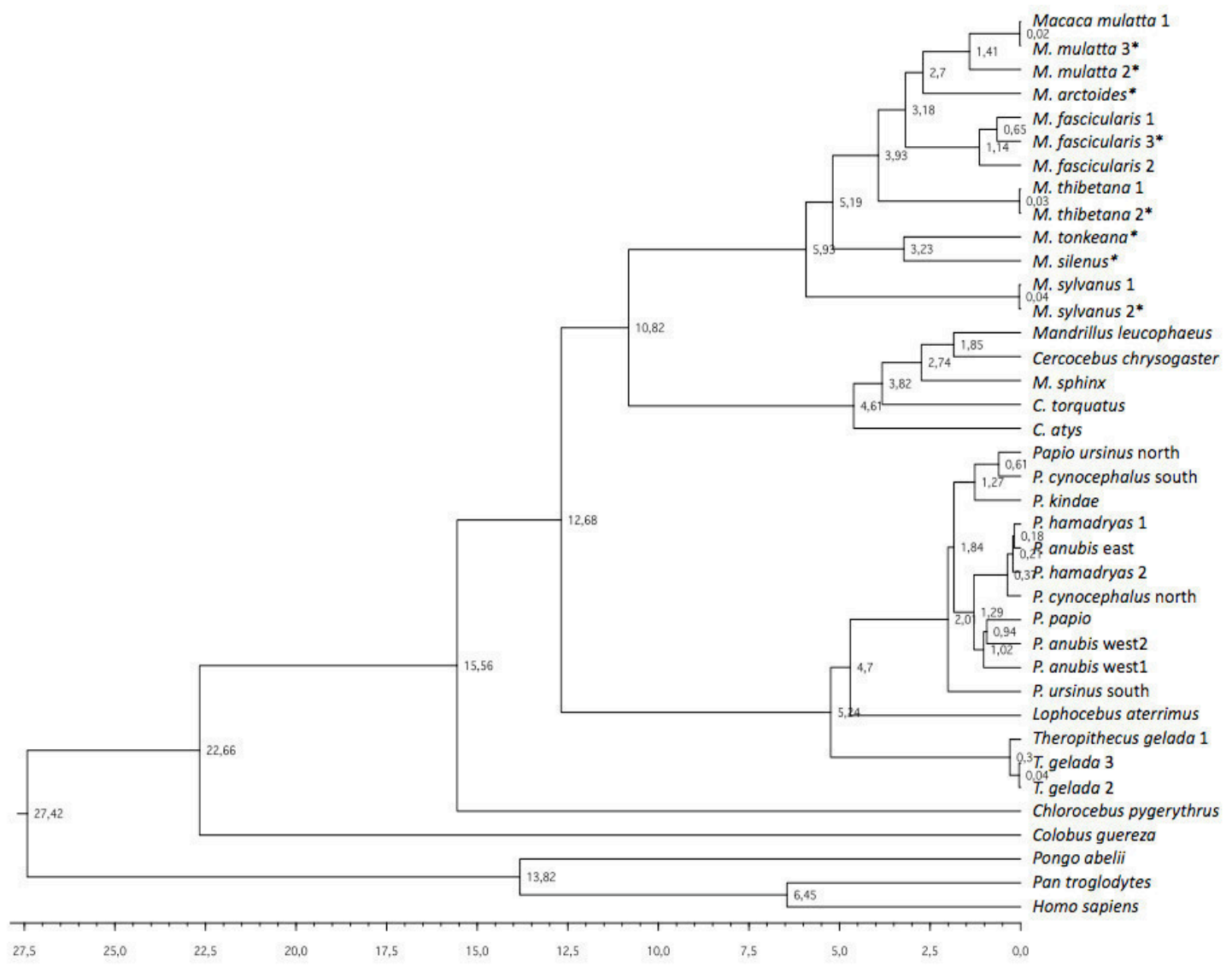

Figure S4. Tree topology including divergence dates as estimated with an uncorrelated relaxed clock model as implemented in PhyloBayes 3.3. Time scale shows million years before present. * $=$ sequences were newly generated in this study.

Table S2. Studied species and individuals along with their GenBank accession numbers.

\begin{tabular}{lc}
\multicolumn{1}{c}{ Species } & Accession no. \\
\hline Macaca sylvanus 1 & AJ309865 \\
M. sylvanus 2 & KJ567054 \\
M. arctoides & KJ567055 \\
M. fascicularis 1 & FJ906803 \\
M. fascicularis 2 & KF305937 \\
M. fascicularis 3 & KJ567052 \\
M. mulatta 1 & AY612638 \\
M. mulatta 2 & KJ567051 \\
M. mulatta 3 & KJ567053 \\
M. thibetana 1 & EU294187 \\
M. thibetana 2 & KJ567056 \\
M. tonkeana & KJ567058
\end{tabular}


M. silenus

Mandrillus leucophaeus

M. sphinx

Cercocebus chrysogaster

C. torquatus $^{1)}$

C. atys

Papio anubis east

$P$. anubis west 1

$P$. anubis west 2

$P$. cynocephalus north

$P$. cynocephalus south

$P$. hamadryas 2

P. hamadryas 1

P. papio

P. kindae

$P$. ursinus north

$P$. ursinus south

Theropithecus gelada 1

T. gelada 2

T. gelada 3

Lophocebus aterrimus

Chlorocebus pygerythrus

Colobus guereza

Pongo abelii

Pan troglodytes

Homo sapiens

\section{KJ567057 \\ JQ257001 \\ KC757403 \\ KC757390 \\ JQ256999 \\ JQ256998 \\ JX946196 \\ JX946198 \\ JX946197 \\ JX946199 \\ JX946200 \\ JX946201 \\ NC001992 \\ JX946203 \\ JX946202 \\ JX946204 \\ JX946205 \\ FJ785426 \\ JQ257000 \\ KC757412 \\ KC757401 \\ EF597500 \\ AY863427 \\ X97707 \\ D38113 \\ X93334}

$\left.{ }^{1}\right)$ designated as Lophocebus albigena in [63], but species identification most likely wrong. Sequences in bold were newly generated in this study.

\section{Chapter 3 no supplementary material}

\section{Chapter 4}

Table SI. Detailed information about studied mtDNA genomes (geographical origin, source, GenBank accession number, sequencing method).

\begin{tabular}{|c|c|c|c|c|c|c|}
\hline species/individual no. & location & coordinates & source & source ID & $\begin{array}{c}\text { accession } \\
\text { no. }\end{array}$ & $\begin{array}{l}\text { sequencing } \\
\text { method }\end{array}$ \\
\hline M. fascicularis 1 & $\begin{array}{l}\text { mainland } \\
\text { Southeast Asia } \\
\text { according to } \\
\text { haplotype } \\
\end{array}$ & unknown & GenBank & & FJ906803 & \\
\hline M. fascicularis 2 & $\begin{array}{l}\text { mainland } \\
\text { Southeast Asia } \\
\text { according to } \\
\text { haplotype }\end{array}$ & unknown & GenBank & & KF305937 & \\
\hline M. fascicularis 3 & Vietnam & unknown & $\begin{array}{c}\text { GenBank/ } \\
\text { Covance }\end{array}$ & & KJ567052 & \\
\hline M. fascicularis 4 & $\begin{array}{l}\text { Bangkok, } \\
\text { Thailand }\end{array}$ & $\begin{array}{c}\text { ca. } 13^{\circ} 45^{\prime} \mathrm{N} \\
100^{\circ} 31^{\prime} \mathrm{E}\end{array}$ & ZSM & $1904 / 05$ & KM851024 & Ion Torrent \\
\hline M. fascicularis 5 & $\begin{array}{l}\text { Bangkok, } \\
\text { Thailand }\end{array}$ & $\begin{array}{c}\text { ca. } 13^{\circ} 45^{\prime} \mathrm{N} \\
100^{\circ} 31^{\prime} \mathrm{E}\end{array}$ & ZSM & $1949 / 1486$ & KM851036 & Ion Torrent \\
\hline
\end{tabular}




\begin{tabular}{|c|c|c|c|c|c|c|}
\hline M. fascicularis 6 & $\begin{array}{l}\text { Tanah Merah, } \\
\text { Kelantan, West } \\
\text { Malaysia }\end{array}$ & $\begin{array}{l}\text { ca. } 5^{\circ} 48^{\prime} \mathrm{N} \\
102^{\circ} 09^{\prime} \mathrm{E}\end{array}$ & MdZain & $\begin{array}{l}\text { Tanah } \\
\text { Merah1 }\end{array}$ & KM851016 & Ion Torrent \\
\hline M. fascicularis 7 & $\begin{array}{l}\text { Terengganu, } \\
\text { Pulau Redang, } \\
\text { West Malaysia }\end{array}$ & $\begin{array}{c}\text { ca. } 5^{\circ} 47^{\prime} \mathrm{N} \\
103^{\circ} 01^{\prime} \mathrm{E}\end{array}$ & MdZain & Redang1 & KM851012 & Sanger \\
\hline M. fascicularis 8 & $\begin{array}{l}\text { Kuala Gula, } \\
\text { Perak, West } \\
\text { Malaysia } \\
\end{array}$ & $\begin{array}{l}\text { ca. } 4^{\circ} 57^{\prime} \mathrm{N} \\
100^{\circ} 32^{\prime} \mathrm{E}\end{array}$ & MdZain & $\begin{array}{l}\text { Kuala } \\
\text { Gula1 }\end{array}$ & KM851018 & Ion Torrent \\
\hline M. fascicularis 9 & $\begin{array}{l}\text { Sepang, } \\
\text { Selangor, West } \\
\text { Malaysia }\end{array}$ & $\begin{array}{c}\text { ca. } 2^{\circ} 49^{\prime} \mathrm{N} \\
101^{\circ} 44^{\prime} \mathrm{E}\end{array}$ & MdZain & Sepang1 & KM851017 & Ion Torrent \\
\hline M. fascicularis 10 & $\begin{array}{l}\text { Kluang, Johor, } \\
\text { West Malaysia }\end{array}$ & $\begin{array}{c}\text { ca. } 2^{\circ} 02^{\prime} \mathrm{N}, \\
103^{\circ} 19^{\prime} \mathrm{E} \\
\end{array}$ & MdZain & Kluang1 & KM851015 & Ion Torrent \\
\hline M. fascicularis 11 & $\begin{array}{l}\text { Batang Kwis, } \\
\text { Medan, Sumatra } \\
\end{array}$ & $\begin{array}{l}\text { ca. } 3^{\circ} 40^{\prime} \mathrm{N}, \\
98^{\circ} 44^{\prime} \mathrm{E}\end{array}$ & ZSM & $1906 / 35$ & KM850999 & Sanger \\
\hline M. fascicularis 12 & Deli, Sumatra & $\begin{array}{l}\text { ca. } 3^{\circ} 35^{\prime} \mathrm{N}, \\
98^{\circ} 40^{\prime} \mathrm{E}\end{array}$ & ZSM & $1921 / 183$ & KM851010 & Ion Torrent \\
\hline M. fascicularis 13 & Deli, Sumatra & $\begin{array}{l}\text { ca. } 3^{\circ} 35^{\prime} \mathrm{N}, \\
98^{\circ} 40^{\prime} \mathrm{E}\end{array}$ & ZSM & $1921 / 187$ & KM851011 & Ion Torrent \\
\hline M. fascicularis 14 & $\begin{array}{l}\text { Kampong Baru, } \\
\text { Sumatra }\end{array}$ & $\begin{array}{c}\text { ca. } 3^{\circ} 33^{\prime} \mathrm{N}, \\
98^{\circ} 41^{\prime} \mathrm{E} \\
\end{array}$ & ZSM & $1906 / 61$ & KM850998 & Sanger \\
\hline M. fascicularis 15 & \begin{tabular}{|l|} 
Sungei Rampalz \\
Serdang, east \\
coast Sumatra \\
\end{tabular} & $\begin{array}{l}\text { ca. } 3^{\circ} 30^{\prime} \mathrm{N} \\
99^{\circ} 00^{\prime} \mathrm{E}\end{array}$ & ZSM & $1905 / 135$ & KM851022 & Ion Torrent \\
\hline M. fascicularis 16 & $\begin{array}{l}\text { Sungei Rampalz } \\
\text { Serdang, east } \\
\text { coast Sumatra }\end{array}$ & $\begin{array}{l}\text { ca. } 3^{\circ} 30^{\prime} \mathrm{N} \\
99^{\circ} 00^{\prime} \mathrm{E}\end{array}$ & ZSM & $1905 / 134$ & KM851035 & Ion Torrent \\
\hline M. fascicularis 17 & $\begin{array}{l}\text { Simpang Bangka, } \\
\text { Sumatra }\end{array}$ & $\begin{array}{c}\text { ca. } 2^{\circ} 18^{\prime} S \\
106^{\circ} 04^{\prime} \mathrm{E}\end{array}$ & ZSM & 1905 & KM851023 & Ion Torrent \\
\hline M. fascicularis 18 & $\begin{array}{l}\text { Simpang Bangka, } \\
\text { Sumatra }\end{array}$ & $\begin{array}{c}\text { ca. } 2^{\circ} 18^{\prime} \mathrm{S} \\
106^{\circ} 04^{\prime} \mathrm{E}\end{array}$ & ZSM & none & KM851034 & Ion Torrent \\
\hline M. fascicularis 19 & Kediri, Java & $\begin{array}{c}\text { ca. } 7^{\circ} 48^{\prime} \mathrm{S} \\
112^{\circ} 15^{\prime} \mathrm{E} \\
\end{array}$ & ZSM & $1911 / 2363$ & KM851031 & Ion Torrent \\
\hline M. fascicularis 20 & Kediri, Java & $\begin{array}{l}\text { ca. } 7^{\circ} 48^{\prime} \mathrm{S} \\
112^{\circ} 15^{\prime} \mathrm{E}\end{array}$ & ZSM & $1911 / 2364$ & KM851021 & $\begin{array}{c}\text { Sanger + lon } \\
\text { Torrent }\end{array}$ \\
\hline M. fascicularis 21 & Kediri, Java & $\begin{array}{l}\text { ca. } 7^{\circ} 48^{\prime} \mathrm{S} \\
112^{\circ} 15^{\prime} \mathrm{E}\end{array}$ & ZSM & $1911 / 2365$ & KM851029 & Ion Torrent \\
\hline M. fascicularis 22 & Kediri, Java & $\begin{array}{c}\text { ca. } 7^{\circ} 48^{\prime} \mathrm{S} \\
112^{\circ} 15^{\prime} \mathrm{E}\end{array}$ & ZSM & $1911 / 2356$ & KM851020 & Ion Torrent \\
\hline M. fascicularis 23 & Kediri, Java & $\begin{array}{c}\text { ca. } 7^{\circ} 48^{\prime} \mathrm{S} \\
112^{\circ} 15^{\prime} \mathrm{E} \\
\end{array}$ & ZSM & $1911 / 2353$ & KM851028 & Ion Torrent \\
\hline M. fascicularis 24 & Lelogama, Timor & $\begin{array}{c}\text { ca. } 9^{\circ} 44^{\prime} \mathrm{S} \\
123^{\circ} 57^{\prime} \mathrm{E}\end{array}$ & ZSM & $1911 / 2104$ & KM851033 & Ion Torrent \\
\hline M. fascicularis 25 & Lelogama, Timor & $\begin{array}{c}\text { ca. } 9^{\circ} 44^{\prime} \mathrm{S} \\
123^{\circ} 57^{\prime} \mathrm{E}\end{array}$ & ZSM & $1911 / 2103$ & KM851026 & Ion Torrent \\
\hline M. fascicularis 26 & Lelogama, Timor & $\begin{array}{c}\text { ca. } 9^{\circ} 44^{\prime} \mathrm{S} \\
123^{\circ} 57^{\prime} \mathrm{E}\end{array}$ & ZSM & $1911 / 2102$ & KM851025 & Ion Torrent \\
\hline M. fascicularis 27 & Lelogama, Timor & $\begin{array}{c}\text { ca. } 9^{\circ} 44^{\prime} \mathrm{S} \\
123^{\circ} 57^{\prime} \mathrm{E}\end{array}$ & ZSM & $1911 / 2105$ & KM851019 & Ion Torrent \\
\hline M. fascicularis 28 & Lelogama, Timor & $\begin{array}{c}\text { ca. } 9^{\circ} 44^{\prime} \mathrm{S} \\
123^{\circ} 57^{\prime} \mathrm{E}\end{array}$ & ZSM & $1911 / 2106$ & KM851027 & Ion Torrent \\
\hline M. fascicularis 29 & Lelogama, Timor & $\begin{array}{c}\text { ca. } 9^{\circ} 44^{\prime} \mathrm{S} \\
123^{\circ} 57^{\prime} \mathrm{E}\end{array}$ & ZSM & $1911 / 2107$ & KM851037 & Ion Torrent \\
\hline M. fascicularis 30 & Kuatnana, Timor & $\begin{array}{c}\text { ca. 9॰50'S, } \\
124^{\circ} 10^{\prime} \mathrm{E} \\
\end{array}$ & ZSM & $1911 / 2108$ & KM851030 & Ion Torrent \\
\hline M. fascicularis 31 & Bokong, Timor & $\begin{array}{l}\text { ca. } 9^{\circ} 58^{\prime} \mathrm{S} \\
124^{\circ} 04^{\prime} \mathrm{E}\end{array}$ & ZSM & $1911 / 2109$ & KM851032 & $\begin{array}{l}\text { Sanger + lon } \\
\text { Torrent }\end{array}$ \\
\hline M. fascicularis 32 & $\begin{array}{l}\text { Lundu, Sarawak, } \\
\text { Borneo }\end{array}$ & $\begin{array}{l}\text { ca. } 1^{\circ} 40^{\prime} \mathrm{N}, \\
109^{\circ} 48^{\prime} \mathrm{E}\end{array}$ & MdZain & Lundu1 & KM851014 & Sanger \\
\hline M. fascicularis 33 & $\begin{array}{l}\text { Sangan, west } \\
\text { coast Borneo }\end{array}$ & unknown & ZSM & $1910 / 1373$ & KM851005 & Ion Torrent \\
\hline M. fascicularis 34 & $\begin{array}{l}\text { Sangan, west } \\
\text { coast Borneo }\end{array}$ & unknown & ZSM & $1909 / 1358$ & KM851007 & Ion Torrent \\
\hline M. fascicularis 35 & $\begin{array}{l}\text { Paian, west coast } \\
\text { Borneo }\end{array}$ & unknown & ZSM & $1909 / 1387$ & KM851009 & Ion Torrent \\
\hline M. fascicularis 36 & $\begin{array}{l}\text { Sungei, Malai, } \\
\text { west coast } \\
\text { Borneo } \\
\end{array}$ & unknown & ZSM & $1910 / 1437$ & KM851003 & Ion Torrent \\
\hline M. fascicularis 37 & $\begin{array}{l}\text { Baeajan, west } \\
\text { coast Borneo }\end{array}$ & unknown & ZSM & $1910 / 1534$ & KM851004 & Ion Torrent \\
\hline M. fascicularis 38 & $\begin{array}{l}\text { Plandjan, central } \\
\text { Borneo }\end{array}$ & $\begin{array}{c}\text { ca. } 0^{\circ} 04^{\prime} \mathrm{N} \\
111^{\circ} 30^{\prime} \mathrm{E}\end{array}$ & ZSM & 1909/766 & KM851002 & Sanger \\
\hline
\end{tabular}


Supplementary

\begin{tabular}{|c|c|c|c|c|c|c|}
\hline M. fascicularis 39 & central Borneo & $\begin{array}{c}\text { ca. } 0^{\circ} 04^{\prime} \mathrm{N} \\
111^{\circ} 30^{\prime} \mathrm{E}\end{array}$ & ZSM & $1909 / 771$ & KM851008 & Ion Torrent \\
\hline M. fascicularis 40 & $\begin{array}{l}\text { Schkadon, } \\
\text { central Borneo }\end{array}$ & $\begin{array}{l}\text { ca. } 0^{\circ} 04^{\prime} \mathrm{N} \\
111^{\circ} 30^{\prime} \mathrm{E}\end{array}$ & ZSM & $1909 / 788$ & KM851006 & Ion Torrent \\
\hline M. fascicularis 41 & $\begin{array}{l}\text { Tawau Hill Park, } \\
\text { Sabah, Borneo }\end{array}$ & $\begin{array}{l}\text { ca. } 4^{\circ} 27^{\prime} \mathrm{N} \\
117^{\circ} 57^{\prime} \mathrm{E} \\
\end{array}$ & MdZain & Tawau Hill1 & KM851013 & Sanger \\
\hline M. fascicularis 42 & Philippines & unknown & Covance & none & KM851001 & Sanger \\
\hline M. fascicularis 43 & Mauritius & unknown & DPZ & 23145 & KM851000 & Sanger \\
\hline M. sylvanus 1 & & & GenBank & & AJ309865 & \\
\hline M. sylvanus 2 & & & GenBank & & KJ567054 & \\
\hline M. arctoides & & & GenBank & & KJ567055 & \\
\hline M. mulatta 1 & & & GenBank & & AY612638 & \\
\hline M. mulatta 2 & China & & GenBank & & KJ567051 & \\
\hline M. mulatta 3 & India & & GenBank & & KJ567053 & \\
\hline M. thibetana 1 & & & GenBank & & EU294187 & \\
\hline M. thibetana 2 & & & GenBank & & KJ567056 & \\
\hline M. tonkeana & & & GenBank & & KJ567058 & \\
\hline M. silenus & & & GenBank & & KJ567057 & \\
\hline Theropithecus gelada & & & GenBank & & FJ785426 & \\
\hline Papio hamadryas & & & GenBank & & NC001992 & \\
\hline Chlorocebus pygerythrus & & & GenBank & & EF597500 & \\
\hline Colobus guereza & & & GenBank & & AY863427 & \\
\hline Pongo abelii & & & GenBank & & X97707 & \\
\hline Pan troglodytes & & & GenBank & & D38113 & \\
\hline Homo sapiens & & & GenBank & & X93334 & \\
\hline \multicolumn{7}{|l|}{$\begin{array}{l}\text { M. fascicularis individuals in } \\
\text { bold were newly generated } \\
\text { for this study }\end{array}$} \\
\hline \multicolumn{7}{|l|}{$\begin{array}{l}\text { ZSM = Bavarian State } \\
\text { Collection of Zoology }\end{array}$} \\
\hline $\begin{array}{l}\text { DPZ = German Primate } \\
\text { Center }\end{array}$ & & & & & & \\
\hline
\end{tabular}


Table SII. Estimated divergence ages in Ma and $95 \%$ credibility intervals (in parentheses) among lineages.

\begin{tabular}{|c|c|}
\hline 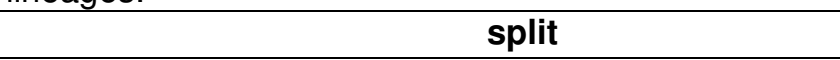 & divergence age (Ma) \\
\hline Cercopithecidae - Hominidae & $28.60(25.31-31.78)$ \\
\hline Pongo - Homo + Pan & $13.82(12.68-14.86)$ \\
\hline Homo - Pan & $6.32(5.73-6.89)$ \\
\hline Colobus - Cercopithecinae & $19.89(16.17-23.87)$ \\
\hline Chlorocebus - Papionini & $12.81(10.59-15.22)$ \\
\hline Theropithecus + Papio - Macaca & $10.90(8.92-12.90)$ \\
\hline Theropithecus - Papio & $4.77(3.87-5.72)$ \\
\hline Macaca sylvanus - other Macaca & $6.10(5.23-6.92)$ \\
\hline Macaca sylvanus 1 - M. sylvanus 2 & $0.04(0.02-0.07)$ \\
\hline Macaca silenus + M. tonkeana - other Macaca & $5.49(4.69-6.34)$ \\
\hline Macaca silenus - M. tonkenana & $3.70(2.80-4.54)$ \\
\hline Macaca thibetana - other Macaca & $4.16(3.47-4.85)$ \\
\hline Macaca thibetana 1 - M. thibetana 2 & $0.04(0.02-0.06)$ \\
\hline Macaca arctoides + M. mulatta - M. fascicularis & $3.42(2.83-4.01)$ \\
\hline M. arctoides - M. mulatta & $3.02(2.42-3.60)$ \\
\hline Macaca mulatta 2 - M. mulatta $1+$ M. mulatta 3 & $1.67(1.19-2.15)$ \\
\hline Macaca mulatta 1 - M. mulatta 3 & $0.02(0.01-0.04)$ \\
\hline Macaca fascicularis Clade A - M. fascicularis Clade B & $1.70(1.36-2.04)$ \\
\hline Clade A: MFAS2+4+5 - MFAS1+3+6-16 & $0.96(0.78-1.16)$ \\
\hline Clade A: MFAS2 - MFAS4+5 & $0.30(0.20-0.41)$ \\
\hline Clade A: MFAS4 - MFAS5 & $0.04(0.02-0.07)$ \\
\hline Clade A: MFAS1+3 - MFAS6-16 & $0.88(0.70-1.06)$ \\
\hline Clade A: MFAS1 - MFAS3 & $0.45(0.34-0.57)$ \\
\hline Clade A: MFAS7 - MFAS6+8-16 & $0.70(0.55-0.84)$ \\
\hline Clade A: MFAS6 - MFAS8-16 & $0.56(0.43-0.68)$ \\
\hline Clade A: MFAS8 - MFAS9-16 & $0.40(0.31-0.49)$ \\
\hline Clade A: MFAS9 - MFAS10-16 & $0.27(0.21-0.34)$ \\
\hline Clade A: MFAS10+14 - MFAS11-13+15+16 & $0.25(0.20-0.31)$ \\
\hline Clade A: MFAS10 - MFAS14 & $0.22(0.16-0.28)$ \\
\hline Clade A: MFAS11-13 - MFAS15+16 & $0.20(0.15-0.25)$ \\
\hline Clade A: MFAS11 - MFAS12+13 & $0.11(0.07-0.15)$ \\
\hline Clade A: MFAS12 - MFAS13 & $0.02(0.01-0.04)$ \\
\hline Clade A: MFAS15 - MFAS16 & $0.04(0.02-0.07)$ \\
\hline Clade B: MFAS24-31 - MFAS17-23+32-43 & $0.93(0.74-1.12)$ \\
\hline Clade B: MRCA MFAS24-31 & $0.03(0.01-0.04)$ \\
\hline Clade B: MFAS19-23 - MFAS17+18+32-43 & $0.87(0.70-1.05)$ \\
\hline Clade B: MRCA MFAS19-23 & $0.01(0.00-0.02)$ \\
\hline Clade B: MFAS43 - MFAS17+18+32-42 & $0.84(0.67-1.02)$ \\
\hline Clade B: MFAS17+18 - MFAS32-42 & $0.61(0.47-0.75)$ \\
\hline Clade B: MFAS17 - MFAS18 & $0.18(0.11-0.25)$ \\
\hline Clade B: MFAS41+42 - MFAS32-40 & $0.33(0.26-0.41)$ \\
\hline Clade B: MFAS41 - MFAS42 & $0.21(0.15-0.28)$ \\
\hline Clade B: MFAS37-39 - MFAS32-35+40 & $0.30(0.24-0.38)$ \\
\hline Clade B: MFAS37 - MFAS38+39 & $0.05(0.03-0.07)$ \\
\hline Clade B: MFAS38 - MFAS39 & $0.03(0.02-0.05)$ \\
\hline Clade B: MFAS32+36 - MFAS33-35+40 & $0.28(0.22-0.35)$ \\
\hline Clade B: MFAS32 - MFAS36 & $0.20(0.14-0.26)$ \\
\hline Clade B: MFAS35 - MFAS3/34+40 & $0.09(0.06-0.13)$ \\
\hline Clade B: MFAS33/34 - MFAS40 & $0.04(0.02-0.07)$ \\
\hline
\end{tabular}




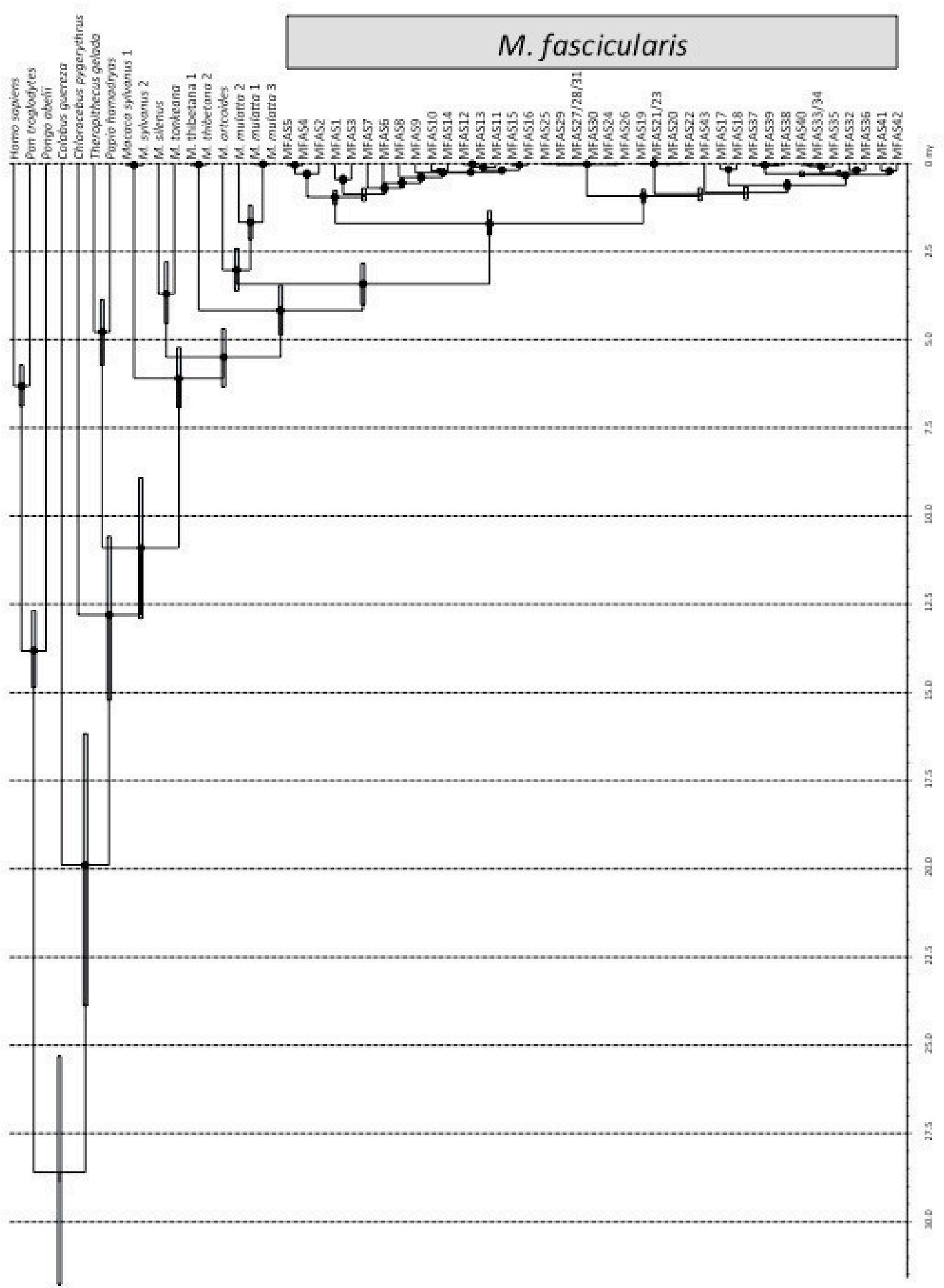

Figure SI. Ultrametric tree showing phylogenetic relationships and divergence ages among 56 unique mtDNA genome sequences. Grey bars indicate $95 \%$ credibility intervals of divergence times and the time scale below shows million years before present. Numbers correspond to IDs in Figs. 1 \& 2 and Supporting Information Table SI. Black dots indicate ML bootstrap support of $>95 \%$ and Bayesian posterior probabilities of 1.0 . 


\section{Danksagung}

Zuerst möchte ich mich herzlich bei Christian Roos und Dietmar Zinner für die geduldige Betreuung und die tatkräftige Unterstützung dieser Doktorarbeit bedanken. Ohne sie wäre diese Arbeit nicht zustande gekommen.

Besonderer Dank geht auch an Christiane Schwarz für die tolle Zusammenarbeit im Labor. Ihr Einsatz hat mir viel Arbeit abgenommen.

Des Weiteren gilt mein Dank Lutz Walter, Markus Brameier, Nico Westphal, Kai Böker, Tanja Haus, Gisela Kopp und allen Mitarbeitern der Abteilung Primatengenetik für ihre Unterstützung und die gute Zusammenarbeit. Hier möchte ich mich besonders bei Jakob Kolleck bedanken, der durch seinen Einsatz im ancient DNA Labor wichtige Daten für diese Arbeit generiert hat. An dieser Stelle sei auch Sabine Hutschenreuther gedankt, die mir geholfen hat, die vielen Makaken in der Zoologischen Staatssammlung München zu beproben. Außerdem ein großes Dankeschön an Rebecca Jürgens für Tipps und Korrekturen sowie motivierende Worte. Besonders bedanken möchte ich mich auch dafür, dass ich im Rahmen meiner Arbeit in der Primatengenetik die Möglichkeit bekommen habe, nach Asien zu reisen und neben Konferenzbesuchen auch einige Primaten in ihrem natürlichen Lebensraum sehen zu können.

Zum Schluss möchte ich natürlich auch meiner Familie, meinen Freunden und besonders Verena danken, die mich immer unterstützt und motiviert haben, auch wenn ich in den letzten Monaten sicher nicht immer leicht zu ertragen war. 


\section{Lebenslauf}

Rasmus Liedigk

Diplom Biologe

Geboren am 19.07.1978 in Westerstede

Staatsangehörigkeit: deutsch

08/2010 - 09/2014 Anfertigung der vorliegenden Dissertation in der Abteilung Primatengenetik am Deutschen Primatenzentrum Göttingen

11/2009-07/2010 Wissenschaftlicher Mitarbeiter am Johann-FriedrichBlumenbach Institut für Zoologie, Georg-AugustUniversität Göttingen.

12/2008-10/2009 Anfertigung der Diplomarbeit „Molecular phylogeny of species groups within the genera Trachypithecus and Semnopithecus" am Deutschen Primatenzentrum Göttingen

10/2003-10/2008 Studium im Fachbereich Biologie, Georg-AugustUniversität Göttingen

Schwerpunkte: Zoologie, Evolutionsbiologie, Naturschutz, Geologie, Abschluss: Diplom 


\section{Eidesstattliche Erklärung}

Ich erkläre hiermit, dass ich diese Dissertation selbstständig und ohne Benutzung anderer als der angegebenen Quellen und Hilfsmittel verfasst habe. Alle den benutzten Quellen wörtlich oder sinngemäß entnommenen Stellen sind als solche einzeln kenntlich gemacht.

Des Weiteren erkläre ich, dass ich mich nicht anderweitig einer Doktorarbeit ohne Erfolg unterzogen habe und dass diese Arbeit in gleicher oder ähnlicher Form noch keiner anderen Prüfungsbehörde vorgelegen hat.

Göttingen, 4.9. 2014 